\title{
Role of DLG-MAGUKs in surface NMDAR localization and its patho-physiological functions
}

\author{
Dissertation \\ for the award of the degree \\ Doctor rerum naturalium (Dr. rer. nat.) \\ of the Georg-August-Universität Göttingen \\ within the doctoral program (CMPB) \\ of the Georg-August University School of Science (GAUSS)
}

submitted by

\section{Tanmoy Samaddar}

from Kolkata, India

Göttingen, March 2014 
Thesis Committee Members:

Dr. Dr. Oliver Schlüter (reviewer)

Molecular neurobiology, European Neuroscience Institute, Göttingen

Prof. Dr. Reinhard Jahn (reviewer)

Dept. of Neurobiology, Max Planck Institute for Biophysical Chemistry, Göttingen

Prof. Dr. Henning Urlaub

Bioanalytical Mass Spectrometry Group, Max Planck Institute for Biophysical Chemistry, Göttingen

\section{Extended committee members}

Prof. Dr. Martin Göpfert

Dept. of Cellular Neurobiology, Schwann-Schleiden Research Centre, Göttingen

C.Dean, PhD

Trans-synaptic Signaling, European Neuroscience Institute Göttingen

Prof. Dr. T.Dresbach

Dept. of Anatomy and Embryology, Center of Anatomy, Göttingen

Date of oral examination: $\mathbf{1 2}^{\text {th }}$ May, 2014. 
I hereby declare that I prepared the PhD thesis "Role of DLG-MAGUKs in surface NMDAR localization and its patho-physiological functions" on my own and with no other sources and aids than quoted.

Tanmoy Samaddar 


\section{Abstract}

The post-synaptic side of an excitatory synapse is characterized by an electron-dense, highly dynamic architecture of proteins, called the post-synaptic density or PSD. PSDs comprises of a diverse group of proteins, from glutamate receptors, scaffolding proteins, signaling molecules and cyto-skeletal elements. Amongst this protein ensemble, the MAGUK family of scaffolding proteins is well known for their roles in synaptic maturation, glutamatergic receptor organization and for serving as platform for post-translational modifications of other proteins in the PSD. PSD-93, one of the lesser-known members of this family of proteins had been reported to have contradictory functions in maintaining AMPA and NMDA receptor levels in different brain regions. In this study, using diverse biochemical approaches in a mouse model, I studied the role of PSD-93 in maintaining surface level of glutamatergic receptors and its patho-physiological significance in glutamate induced neuronal toxicity.

By using surface protein cross-linking approach, I could demonstrate that in the layer $2 / 3$ visual cortex of a PSD-93 knockout mice, there is a reduction in the surface level of both NMDA receptor subunits GluN2A and GluN2B. Additionally, I could also demonstrate that this reduction in surface level of GluN2B is caused independent of the change in phosphorylation of the GluN2B-Y1472 site, possibly through other phosphorylation mechanism.

Since, NMDA receptors are classically linked with glutamate-induced neurotoxicity during ischemic brain damage, I looked at the level of neurotoxicity in PSD-93KO cortical cultures. I established a NMDA challenge protocol that enabled neuronal apoptosis and I could show an almost $80 \%$ reduction in cortical cell death, when PSD93 is absent. This form of NMDA neurotoxicity was largely mediated via the GluN2B subunit of NMDA receptors and the neuro-protection effect in PSD-93KO was mediated by perturbation of the P38 MAPK pathway of apoptosis induction.

PSD-95 had been classically reported to link NMDA receptor activation with downstream apoptotic signaling cascade. I could show that deletion of both PSD-93 and 
PSD-95 showed equal degrees of neuro-protection at lower doses of NMDA challenge whereas at higher doses, only PSD-93 retained its neuro-protective effects.

Overall, my data helps to understand the role of PSD-93 in maintaining surface NMDA receptors levels in cortical neurons and linking them to their pathophysiological functions. 
"Happiness can be found, even in the darkest of times, if one only remembers to turn on the light."

Prof. Albus Percival Wulfric Brian Dumbledore. 


\section{Acknowledgement}

First of all, I would like to express my heart-felt gratitude to my supervisor, Dr. Dr. Oliver Schlüter, for giving me the chance to be a part of his research group and have faith and trust in my abilities through-out these years. You are not only an excellent scientific mentor, but your overall attitude and optimism is highly infectious. This work was only possible because of the amount of freedom you gave me in thinking independently, listening tirelessly to the craziest of ideas and always giving me a pick-me-up when morals ran low.

I am grateful to Prof. Dr. Reinhard Jahn and Prof. Dr. Henning Urlaub for being a part of my thesis committee, for the time they devoted to my project and for all the very enriching discussions we had during our meetings.

I thank my collaborator Dr. Miroslav Nikolov, Monika Raabe and Prof. Dr. Henning Urlaub for the mass spectrometric analysis and the discussions. I am grateful to the AGCT DNA Core Facility for providing high-quality oligonucleotides and for the timely sequencing. I would like to thank Veena J and Priyanka Saini from Prof. Dr. Matthias Dobblestein group, and Dr. Tolga Soykan and Bekir Altas from Prof. Dr. Nils Brose for sharing antibodies and chemicals, whenever was needed.

It was a pleasure being part of the lab seminar in the Brose department in MPI-EM and sharing scientific ideas with you all. Each one of you made me feel at home!

In all sense, the lab becomes our second home during our research career, and truly so because of the great scientific family that I was very lucky to have in my lab. Thank you for sharing ideas, laughs, tantrums and much more during the past few years. Sandra, a big thank you not only for the excellent scientific help that you provide dayin and day-out, but also for being the unbelievably loving and caring person that you are. Yanling, it had been great to know you. Steph, Huang, Lei, Man Ho, Peter, Agatha, the lab would never be so much fun without each of you wonderful guys. Derya, you had been a great senior and an even better friend. Our lunch and coffee sessions with you, Charlie, Plinio, Avani and Markus will be a treasured memory to me. Plinio, I started my journey in the lab with you and I could not have hoped for a better companion than you. You had been more than a friend and I am sure we are going to 
stay that way forever more. Avani, you have been my one desi connection in the lab and I am so proud for that.

Ania, it had been a pleasure knowing you. You are not only my most favorite labmate, but the one person I will miss the most no matter where I am. Thanks for being there. Markus, you had been a terrific friend and a great support since the time I have known you. I wouldn't trade the time I spend with both of you guys for anything in the world.

Having no brother myself, I never knew what it is like to have one. But Vincenzo, thanks to you, I know how it feels exactly, and let me tell you, it's awesome! The genuineness of your love for me, I am sure, will never fade away. I am unimaginably thankful to two guys, Alex and Stella, for turning a WG into a home. Every moment with you guys, have been like with my own family. You guys are just the best and I love you both so much!

Christian, you are the most amazing friend that I have made over here, thanks for being there no matter what. This thesis would not have seen the light of the day, without the amazing help that you've always provided. You are a real gem of a person and I am so glad to have met you.

I can't thank enough my Indian family in Göttingen. Somdatta, you know how much I love you. Iswar, Aniket and Mayur, the three "dudes", you guys had been the life line of our group. Sonia di and Anjali, you were the two sisters, that I never had and without you life would have been undeniably boring. Thanks for being there always. Upasana, you are one of the most caring soul I know, so stay the way you are, always. Jatin, Sashi, Shweta, Varun, Shalaka, Sarath, Pawan, Pranav, Vidya, Anurag, I can go on and on about each one of you, but long story short, you guys rock! The kinder group, Vinita, Ankit, Avani, Veena, Chaitali, Akansha, Heena, Sumir, Priyanka, Soham, you are as much a family to me as it can be. Thank you always for making me feel welcome in your midst.

I cannot thank enough my IISc friends, especially, Gauravi, Debjani, Grace, Shruti, Geetha, Shataparna, Sahana, Namrata, and the whole Champistanu batch. Shiv and Anuj, you two were, are and will always be my best buddies. I can not be more thankful for getting you as a friend. Vaishakhi, I feel the same kinship for you as for my own family. Tinni and Kinshuk, thanks for being the best cusion life had offered to me. 
I have a great support in my brother, Sanket. I would not be who I am, without you. Thank you for being the best person in the whole world!!

I have a very loving family. Thank you Maa and Baba, you guys are the reason why I can go through life with my head held high. Your unconditional love is my biggest strength and support.

Last but not the least, I would like to thank GOD, who helps me everyday to sail through all the troubled times of life. There might have been a scratch or a bruise, but you know how to heal them without a lasting scar and I thank you for that with all my heart. 


\section{Contents}

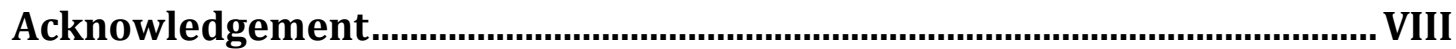

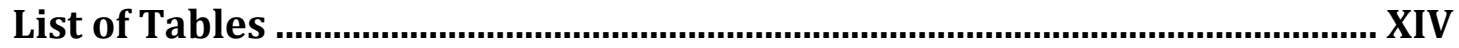

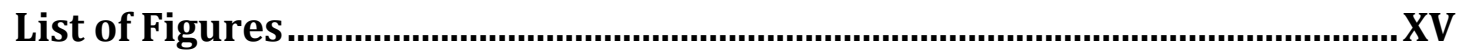

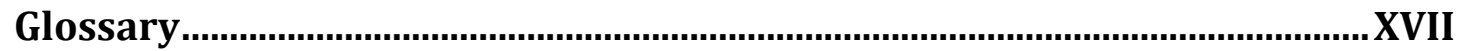

1 Introduction

1.1 Synaptic Transmission .........................................................................

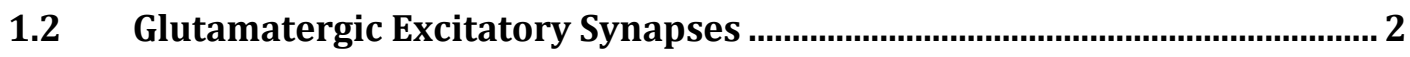

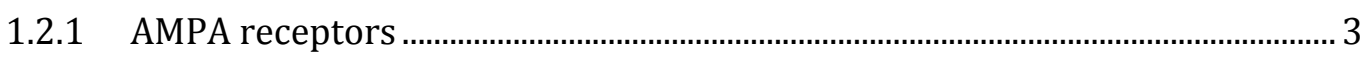

1.2.2 NMDA receptors........................................................................................................ 4

1.2.3 Scaffolding proteins............................................................................................................

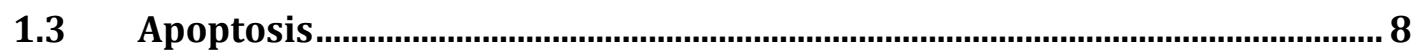

1.3.1 Mechanism .................................................................................................................. 8

1.4 NMDA Receptors: balancing neuro-protection and neurotoxicity............13

1.4.1 GluN2A receptors in neuro-protection ……………………………………….....14

1.4.2 GluN2B in neuronal death and neuro-degeneration ........................................15

1.5 NMDA receptors, dlg-MAGUKs and Ischemia ............................................16

1.6 Scope of the Thesis..................................................................................................17

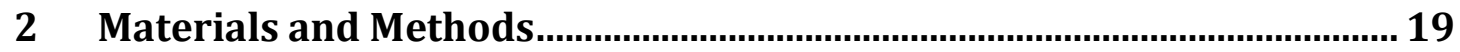

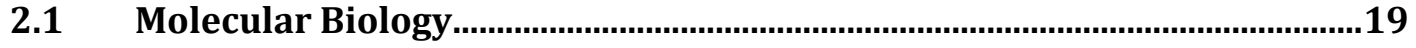

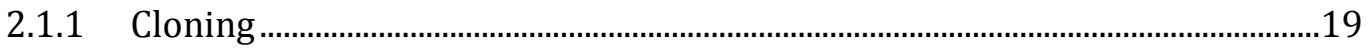

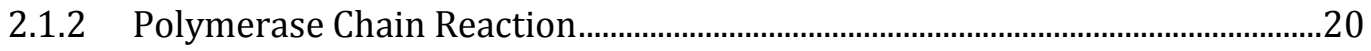

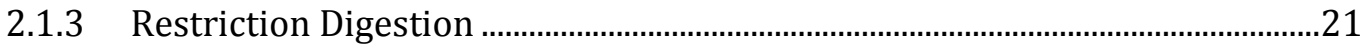

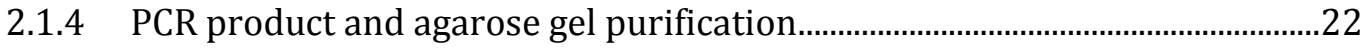

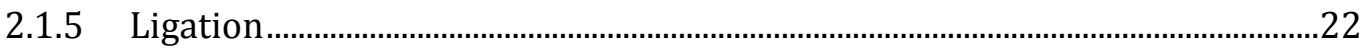

2.1.6 Transformation .....................................................................................................23

2.1.7 Plasmid Preparation.........................................................................................................23

2.1.8 Genotyping .....................................................................................................

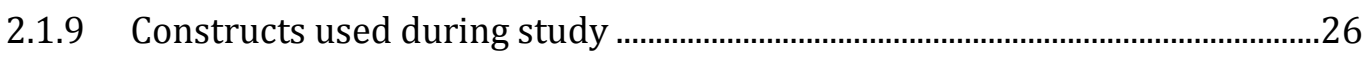

$2.2 \quad$ Cell Culture .............................................................................................................27

2.2.1 HEK293T cell line ........................................................................................................27 


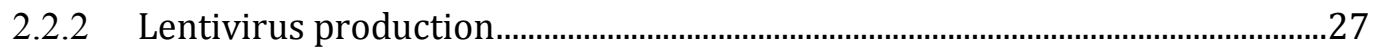

2.2.3 Dissociated hippocampal culture .......................................................................29

2.2.4 Lentiviral infection of dissociated neuronal culture.......................................30

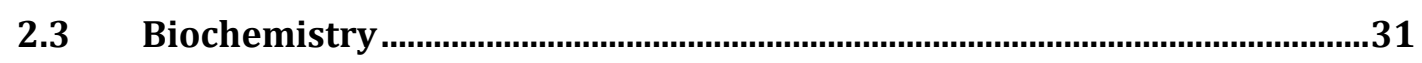

2.3.1 Western Blotting sample preparation from dissociated neuronal culture..31

2.3.2 Co-Immunoprecipiation Assay ..........................................................................31

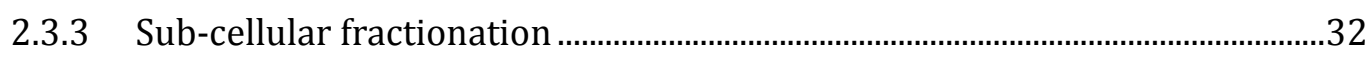

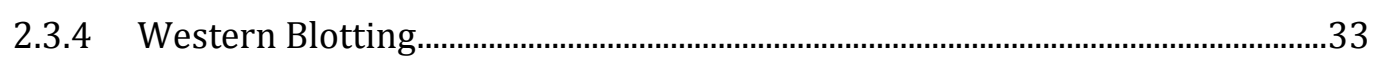

2.3.5 Antibodies used for Immunoblotting..................................................................35

2.3.6 Chemical Cross-linking of Cell surface proteins ....................................................36

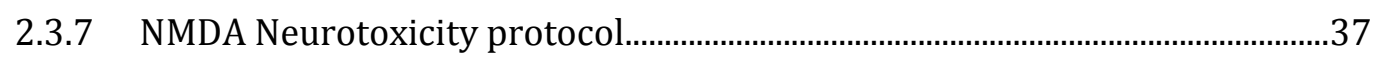

2.3.8 Propidium Iodide staining ....................................................................................

2.3.9 Drugs Used in cell culture and acute visual cortex punches..............................38

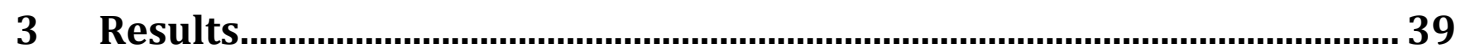

3.1 Only the surface levels but not the total level of NMDA receptors are reduced in PSD-93 knockout visual cortex synapses .................................................40

3.2 Surface levels of AMPA receptors remain unchanged in PSD-93 knockout

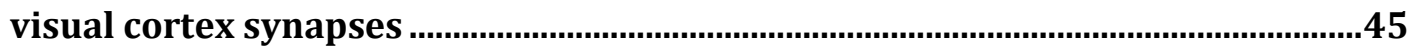

3.3 PSD-93 physically interacts with NMDA receptors in vivo..........................46

3.4 Surface level of phospho-GluN2B subunit is reduced in PSD-93 knockout

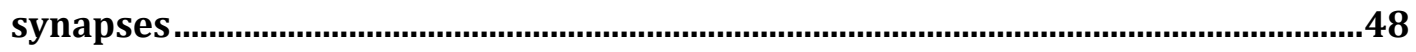

3.5 The level of GluN2B receptor is reduced in PSD-93 cortical but not in hippocampal cultures .........................................................................................50

3.6 Linking PSD-93, NMDA receptors and Apoptosis..........................................55

3.6.1 NMDA receptors are required for maintaining a balance between Neuronal

Cell survival and neuronal Apoptosis................................................................................55

3.6.2 PSD-93 deletion causes reduced neuronal mortality in cortical cells upon NMDA challenge.

3.6.3 Over-expression of selected PSD-93 isoform can rescue the pro-apoptotic effect of PSD-93.

3.6.4 GluN2B is the major contributing NMDA receptor subunit for the NMDA mediated neurotoxicity

3.6.5 P38 MAPK activation profile during NMDA neurotoxicity challenge experiment. .. .70

3.6.6 PSD-93 show reduced levels of active P38 during NMDA challenge .72 
3.6.7 PSD-93KO cultures have lower level of active P38 due of higher level of active STEP.

3.7 Comparing the neuro-protection due to the loss of PSD-93 and PSD-95 against NMDA mediated neurotoxicity in cortical neurons

3.7.1 PSD-95 deletion also causes a reduction in cortical culture mortality but to a lesser extent than PSD-93 deletion

3.7.2 Deletion of PSD-93 has a more severe effect on NMDA mediated neurotoxicity than deletion of PSD-95

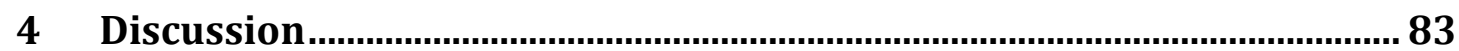

4.2 PSD-93 is required for NMDA induced neurotoxicity . .87

4.3 Loss of PSD-93 is more neuro-protective than PSD-95 in cortical synapses.

4.4 Conclusion and outlook. .97

5 References .99

6 Curriculum Vitae 


\section{List of Tables}

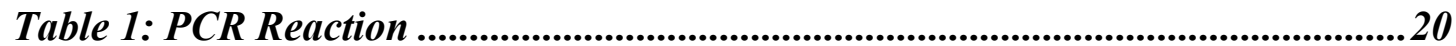

Table 2: Restriction Digestion Reaction ...........................................................21

Table 3: Ligation Reaction ........................................................................................22

Table 4: Primers used for wild-type and PSD-93 knockout animal genotyping......24

Table 5: PCR mix used for wild-type and PSD-93 knockout animal genotyping ....25

Table 6: PCR program for wild-type and PSD-93 knockout animal genotyping.....26

Table 7: DNA mix for lentivirus production.....................................................28

Table 8: Recipies for Bis-Tris gels used for Western blotting...................................33

Table 9: List of antibodies used for immunoblotting.....................................................35

Table 10: Drugs used in cell culture and acute visual cortex punches .....................38 


\section{List of Figures}

Figure 1: Domain organization of a DLG-MAGUK and its interacting partners,

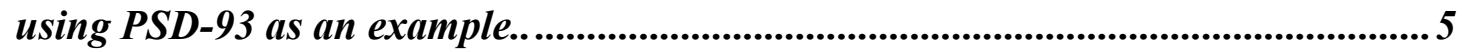

Figure 2: The extrinsic pathway of apoptosis....................................................9

Figure 3: Intrinsic pathways for neuronal apoptosis............................................... 12

Figure 4: PSD-93KO has reduced surface NMDA receptor levels............................42

Figure 5: Total level of NMDA receptors remain unchanged in PSD-93KO

animals. 44

Figure 6: PSD-93KO showed no significant reduction in surface AMPA receptor levels..

Figure 7: PSD-93 showed interaction with both GluN2A and GluN2B proteins in vivo.

Figure 8: PSD-93KO has reduced level phosphorylated GluN2B on the cell surface. 49

Figure 9: PSD-93KO has reduced surface NMDA receptor levels in cortical

culture. 51

Figure 10: PSD-93KO has no change in surface NMDA receptor levels in hippocampal culture. 53

Figure 11: PI staining of NMDA challenged cortical cultures shows cell death.....56

Figure 12: Dose-response curve of NMDA treatment on cortical neurons. 58

Figure 13: Time course for NMDA treatment on cortical neurons..........................60

Figure 14: PSD-93KO cultures have reduced mortality upon NMDA challenge... 62 Figure 15: Over-expression of selected PSD-93 isoform rescues NMDA mediated neurotoxicity. 65 
Figure 16: GluN2A vs. GluN2B contribution in NMDA mediated neurotoxicity.... 68

Figure 17: P38 MAPK activation curve during NMDA challenge experiment. ...... 71

Figure 18: PSD-93KO has reduced level of active P38 MAPK during NMDA

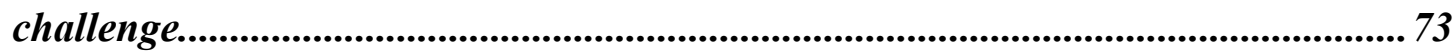

Figure 19: PSD-93KO has elevated levels of active STEP during NMDA challenge

Figure 20: Knockdown of PSD-95 causes reduction in NMDA mediated

neurotoxicity. 78

Figure 21: Deletion of PSD-93 provides better protection against NMDA induced neurotoxicity than deletion of PSD-95. 81

Figure 22: NMDA mediated activation of intrinsic apoptotic pathway in wild-type neurons. 92

Figure 23: In PSD-93KO animals, NMDA mediated activation of apoptosis is blocked. 


\section{Glossary}

ACSF

AMPAR

APV

BCA

CNS

DIV

DNA

E. coli

EPSC

GABA

GK

HEK

Hsp

IP

$\mathrm{KO}$

LTD

LTP
Artificial cerebrospinal fluid

a-amino-3-hydroxyl-5-methyl-4-isoxazole-propionate (AMPA) receptor

(2R)-amino-5-phosphonovaleric acid

Bicinchoninic acid assay

Central Nervous System

Day in vitro

Deoxyribonucleic acid

Escherichia coli

Excitatory postsynaptic current

Y-Aminobutyric acid

Guanylate kinase

Human Embryonic Kidney

Heat shock protein

Immunoprecipitation

Knockout

Long Term Depression

Long Term Potentiation 


\begin{tabular}{|c|c|}
\hline MAGUK & Membrane associated guanylate kinase \\
\hline MAPK & Mitogen-Activated Protein Kinase \\
\hline $\mathrm{n}$ & Number of experiment \\
\hline NBQX & $\begin{array}{l}\text { 2,3-Dioxo-6-nitro-1,2,3,4-tetrahydrobenzo[f]quinoxaline-7- } \\
\text { sulfonamide }\end{array}$ \\
\hline NMDAR & N-methyl-D-aspartic acid (NMDA) receptor \\
\hline n.s. & Non-significant \\
\hline PBS & Phosphate buffered saline \\
\hline PCR & Polymerase Chain Reaction \\
\hline PDZ & Postsynaptic density-95/Discs large/zona occludens-1 \\
\hline PFA & Paraformaldehyde \\
\hline PKA & Protein Kinase A \\
\hline PSD & Post Synaptic Density \\
\hline PSD-93 & Postsynaptic density protein-93 \\
\hline PSD-95 & Postsynaptic density protein-95 \\
\hline PVDF & Polyvinylidene fluoride \\
\hline $\mathrm{rb}$ & Rabbit \\
\hline RIPA & Radioimmunoprecipitation assay buffer \\
\hline RNAi & RNA interference \\
\hline Sap97 & Synapse-associated protein 97 \\
\hline Sap102 & Synapse-associated protein 102 \\
\hline
\end{tabular}


SDS-PAGE SDS-Polyacrylamid gel electrophoresis

SH3

Src homology 3

shRNA

Short-hairpin RNA

STEP

Striatal-enriched Tyrosine Phosphate

TAE

Tris-acetate-EDTA

TARP

Transmembrane AMPAR regulatory proteins

TBS

Tris-buffered saline

WT

Wild-type 


\section{Introduction}

Human brain comprises of over hundred billion neurons. More than hundred years ago (1886-1890) it was recognized by neuro-anatomist including His, Forel and

Ramón Y Cajal, that nerve cells or neurons are independent units capable of selforganized communications. Neural communication takes place between two neurons through highly specialized structures known as synapses. Each neuron, in human brain, on an average, have between 10,000-100,000 synapses, making the total number of synapses in a brain close to an astounding $10^{15}$.

\subsection{Synaptic Transmission}

Synapses are divided into electrical and chemical types. Electrical synapses are reciprocal pathways for ionic current flow, between adjacent neurons separated by only 2-3 $\mathrm{nm}$ gaps (gap junctions). Due to their very rapid activity, electrical synapses are capable of mediating synchronization and spike activity among neuronal clusters.

However, the majority of synapses in the brain are chemical synapses. They are more complex structures with a much higher capacity of having their synaptic transmission strength fine-tuned, based on their previous activity (synaptic plasticity). A chemical synapse has a pre-synaptic neuron separated from its post-synaptic counterpart by a gap of 20-30 nm, called the synaptic cleft. Transmissions at chemical synapses occurs when an action potential reaches the pre-synaptic terminal causing a change in the membrane potential and opening of the voltage-gated $\mathrm{Ca}^{2+}$-channels. A rapid and transient increase in the $\mathrm{Ca}^{2+}$ concentration in the presynaptic terminal causes neurotransmitter containing synaptic vesicles to fuse with the membrane and release their content in the synaptic cleft. The neuro-transmitter diffuses across the cleft to reach and bind to specific receptors on the post-synaptic membrane. This causes the postsynaptic channels to open and allow the flow of ions in or out of the post-synaptic cell. This eventually alters the conductance and hence membrane potential of post- 
synaptic neurons causing them to fire an action potential. That is how information flows from one neuron to the next one.

Depending on the type of neuro-transmitter released from the pre-synaptic terminal, neurons can be excitatory, inhibitory or modulatory in nature. In general, one neuron has one kind of neuro-transmitter: excitatory or inhibitory, while the modulatory neuro-transmitters pair up with either of them. An excitatory synapse is one, where an action potential in the pre-synaptic neuron increases the probability of an action potential in the post-synaptic neuron. The neuro-transmitter glutamate is involved in an excitatory synapse. An inhibitory synapse, on the other hand, helps causing change in the post-synaptic membrane in such a way, that it becomes more difficult for the neuron to fire an action potential. GABA and glycine are the inhibitory neurotransmitters.

\subsection{Glutamatergic Excitatory Synapses}

Excitatory synapses in the brain are characterized by the neuro-transmitter glutamate, that can activate three classes of ionotropic receptors: AMPA, NMDA and kainate. All three classes of receptors are assembled as hetero tetrameric complexes. Addition-

ally, glutamate can also activate G-protein coupled metabotropic receptors, which do not conduct ions but triggers in intra-cellular signaling cascades. Excitatory synapses are formed on dendritic protrusions called spines. A spine head at an excitatory synapse is typically characterized by a complex and highly dynamic architecture of proteins called the post-synaptic density (PSD) (Sheng and Kim, 2011). The PSD is an electron-dense organization of proteins ranging from glutamate receptors, cell adhesion molecules, cytoskeletal elements as well as downstream signaling enzymes. The general role of a PSD is to cluster the post-synaptic receptors efficiently opposite a pre-synaptic neuro-transmitter release site (active zone) and then couple the activation of the post-synaptic receptors with down-stream signaling cascades. 


\subsubsection{AMPA receptors}

AMPA receptors are known to exist as tetrameric assemblies of GluA1-GluA4. The different allele of this receptor differs mainly in their C-terminal part and bind different interacting partners (Hollmann and Heinemann, 1994; Malinow and Malenka, 2002). GluA2 is a key subunit in defining the properties of AMPA receptors. It gets post-translationally modified and renders the channel $\mathrm{Ca}^{2+}$-impermeable. AMPA receptors that lack the GluA2 subunits are susceptible to be blocked by polyamines at their C-terminal end and hence cannot pass current through them at positive membrane potential. Thus for these receptors, when the current to voltage relationship is plotted experimentally, they are inwardly rectifying in nature (Hollmann and Heinemann, 1994; Hume et al., 1991; Jonas and Burnashev, 1995; Koh et al., 1995). The GluA4 subunit, whereas, determines the desensitization kinetics of the AMPA receptors (Mosbacher et al., 1994).Besides their roles in channel properties, the subunit composition also influences the trafficking of these receptors as well (Bredt and Nicoll, 2003; Malinow and Malenka, 2002).

When the abundance of the different subunit compositions were examined, it was found that cortical pyramidal neurons in the Layer 2-4 principally are $\mathrm{Ca}^{2+}$ impermeable, i.e., contains GluA2 subunit and the expression pattern for both GluA2 and GluR3 subunits in these cells were similar. Further it was found out that most cortical pyramidal cells contain the GluA1-GluA2 combinations and highly $\mathrm{Ca}^{2+}$ impermeable (Kondo et al., 1997). In hippocampus, AMPA receptors are either dimers of GluA1-GluA2 (termed GluA1/2) or that of GluA2-GluR3 subunits (termed GluA2/3) (Wenthold et al., 1996). It is suggested that the GluA2/3 AMPA receptors are mainly responsible for basal synaptic transmission and are continuously recycled into the synapse on an average timescale of $15 \mathrm{~min}$, whereas, the GluA1/2 AMPA receptors are more crucial during the induction of LTP into the synapses (Passafaro et al., 2001; Shi et al., 2001). However recent evidences had shown it that $81 \%$ of the synaptic pool of AMPA receptors is comprised of the GluA1/2 and only $15 \%$ by the GluA2/3 AMPA receptors (Lu et al., 2009). In the extra-synapse AMPA receptors are entirely GluA1/2 containing. 
The presence of homomeric GluA1 receptors that are $\mathrm{Ca}^{2+}$-permeable are debated widely ( $\mathrm{Lu}$ et al., 2009; Wenthold et al., 1996). Some believe that $\mathrm{Ca}^{2+}$-permeable GluA1-homomeric AMPA receptors are inserted during LTP (He et al., 2009; Plant et al., 2006), others argue against the existence and essentiality of homomeric GluA1 receptors for LTP (Adesnik and Nicoll, 2007; Bagal et al., 2005). Recent publications have indicated that $\mathrm{Ca}^{2+}$-permeable AMPA receptors might be needed to increase the synaptic strength of AMPA receptors without altering the number of AMPA receptors, especially during drug related memory consolidation (Argilli et al., 2008; Bellone and Lüscher, 2006; Conrad et al., 2008). Thus subunit compositions and their spatio-temporal relevance of AMPA receptors, is still being researched.

\subsubsection{NMDA receptors}

NMDA receptors are one of the principle glutamate receptors on the post synaptic side of the membrane and exists functionally as a tetrameric complex of GluN1 with GluN2A and GluN2B mainly (Andersen et al., 2000; Wenzel et al., 1997). GluN1 is the mandatory subunit in this tetramer as it provides the binding site for the co-agonist glycine or D-serine (Wenthold et al., 2003) and can pair up with either GluN2A or GluN2B, which contain the binding site for glutamate. In hippocampus during early stages of development GluN2B is almost solely expressed but during synaptic maturation the levels of GluN2A goes up. This change in abundance in subunits is called the GluN2B to GluN2A developmental switch (Liu et al., 2004). The GluN2 subunit also governs the channel kinetics of these receptors and the presence of GluN2B causes the channel to have 3-4 times slower decay time (Monyer et al., 1994).

NMDA receptors are co-incidence detectors. At resting potential the channel pore is blocked by $\mathrm{Mg}^{2+}$. Only when the pre-synaptic neuron releases neuro-transmitter and this can sufficiently depolarize the post-synaptic neuron beyond a threshold this $\mathrm{Mg}^{2+-}$ block is lifted and the channel can allow influx of ions. They are highly permeable to $\mathrm{Ca}^{2+}$ as well as $\mathrm{Na}^{+}$and $\mathrm{K}^{+}$ions. 


\subsubsection{Scaffolding proteins}

On the post-synaptic side of the synapse, just below the membrane, lies a class of scaffolding proteins, which are responsible for maintaining the complex protein architecture of the post-synaptic density. This group of proteins is called the DLGMAGUK family and it comprises of four members: chapsyn-110/PSD-93/dLg2, PSD95/SAP90/dLg4, SAP102/dLg3 and SAP97/dLg1. DLG-MAGUKs are known to share a common protein interaction motif. It comprises of three post-synaptic density95/Discs large/Zona Occuludens-1(PDZ) domains, Src homology 3 (SH3) domain and one enzymatically inactive guanylate kinase (GK) domain (Kim and Sheng, 2004).The PDZ domains interact with the c-terminal end of many proteins like GluN2 subunits (Niethammer et al., 1996)

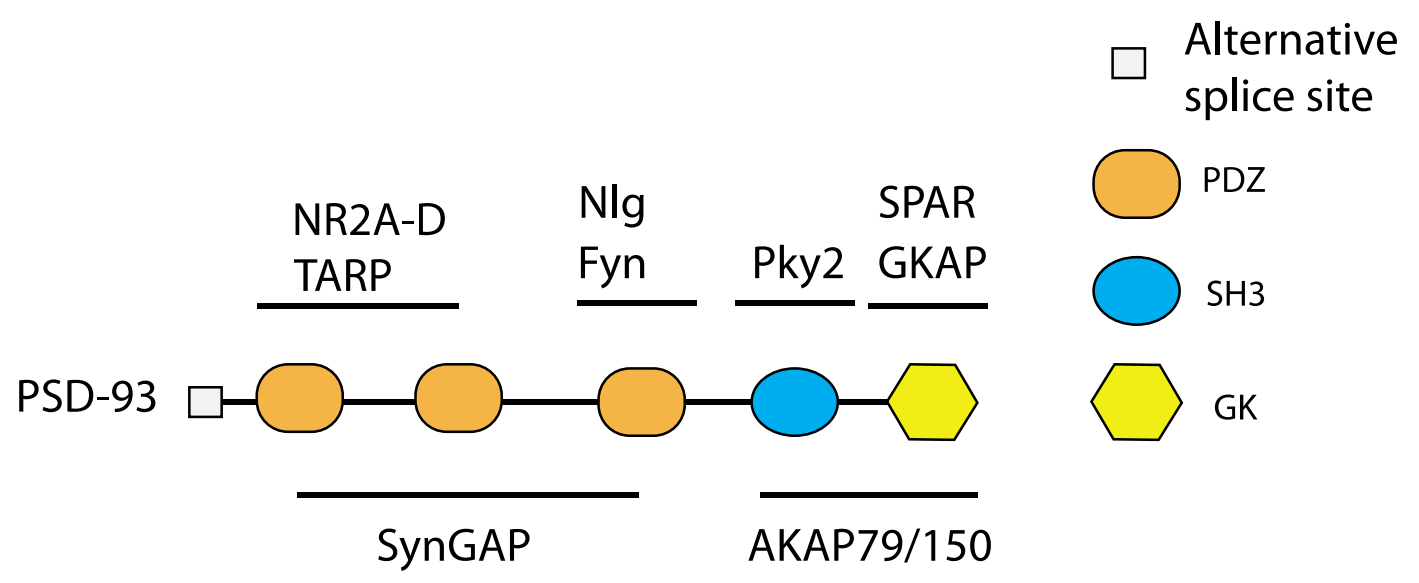

Figure 1: Domain organization of a DLG-MAGUK and its interacting partners, using PSD-93 as an example. The PSD-MAGUK protein has three PDZ domains (shown in light brown), a SH3 (shown in blue) and a GK (shown in yellow) domain. The different $\mathrm{N}$-terminal isoforms are generated by the alternative splice site (shown in white) of the protein. The different known interacting partners had been mentioned and the bar represents the specific domain (s) that they interact with.

Despite their modular similarity and partially redundancy in certain functions, PSDMAGUKs have distinct N-terminal regions due to independent transcription start site, which set them functionally apart from each other. 


\subsubsection{PSD-95 and its synaptic properties}

PSD-95/dLg4 is the best characterized member of the dlg-MAGUK family. It has two N-terminal isoforms PSD-95 $\alpha$ and PSD-95 $\beta$. The N-terminal of PSD-95 $\alpha$ isoform is similar to the SAP97 $\alpha$ isoform (Schlüter et al., 2006) and has a cysteine doublet that's a substrate for palmitoylation (Topinka and Bredt, 1998). Palmitoylation is a special post-translational modification of conjugating a 16-C palmitic acid onto a cysteine residue by formation of a thio-ester bond. One interesting point about this modification is that its labile and reversible and can happen faster than the half-life of a protein (Fukata and Fukata, 2010). Palmitoylation of PSD-95 targets it to the PSD and helps in the stabilization of AMPA receptors at the synapse (Craven et al., 1999). PSD-95 is also known to play a role in synaptic development as over-expression of PSD-95 leads to faster maturation of excitatory synapse (El-husseini, 2014).

In hippocampus, the principle isoform of PSD-95 is the $\alpha$ isoform. It interacts with AMPA receptors via the TARPs and helps in their synaptic trafficking and stability (Bats et al., 2007; Dakoji et al., 2003; Fukata and Fukata, 2010).

Studies with genetic knockouts of PSD-95 have indicated that PSD-95 has no effect on AMPA and NMDA mediated basal synaptic transmission (Migaud et al., 1998) indicating a synaptic compensation for PSD-95 by other MAGUKs (Bonnet, 2011) but one report also claims that absence of PSD-95 in the knockout mice caused an increase in the number of AMPA silent synapses (Béïque et al., 2006). Overexpression of PSD-95 however enhances AMPA receptor transmission (Elias et al., 2006a; Nakagawa et al., 2004; Schlüter et al., 2006; Schnell et al., 2002).

This ideas together advocate for the role of PSD-95 as a molecular slot protein for continues recycling of synaptic AMPA receptors (Schnell et al., 2002; Shi et al., 2001). But studies on the PSD-95KO showed a contrasting increase in LTP whereas LTD was abolished (Béïque et al., 2006; Migaud et al., 1998). Combined this indicates that PSD-95 is not directly required for AMPA receptor entry in synapse during LTP. Rather PSD-95 might act a signaling scaffold to link NMDA receptor activation 
with the downstream signaling cascade as well as act as a synaptic gate-keeper that limits the entry of AMPA receptors to synapses (Schlüter et al., 2006). Hence, in PSD-95KO animals AMPA receptors can access the synapse unhindered and thus cause an increase in the LTP.

Though the NMDA receptor synaptic localization or functions in basal synaptic transmission are not compromised in PSD-95KO animals (Migaud et al., 1998), a higher percentage of GluN2B containing NMDA receptors are increased indicating the need for PSD-95 in the GluN2B to GluN2A developmental switch (Béïque et al., 2006). Most group reports that neither over-expression, nor RNAi mediated knockdown of PSD-95 had alters NMDA synaptic current levels (Béïque and Andrade, 2003; Ehrlich and Malinow, 2004; Elias et al., 2006a; Nakagawa et al., 2004; Schlüter et al., 2006; Schnell et al., 2002; Stein et al., 2003), Futai et al (2007) claims to see increase in NMDA receptor EPSCs following PSD-95 over-expressions.

\subsubsection{PSD-93 and its synaptic properties}

Much about the role of PSD-93 is not very clear and is getting slowly unraveled. One reason for this being that PSD-93 is not as abundant as the rest of the DLG-MAGUKs and its functions are dependent on distinct brain region. PSD-93 knockout animals do not show any changes in synaptic transmission in cerebellum or motor behaviors (McGee et al., 2001a). In the cerebellum neurons, synaptic neurons could assemble and organize normally indicating that PSD-93 does not have any role in synaptic development (McGee et al., 2001a). But when spinal dorsal horn neurons were cultured from PSD-93KO animals, the surface expression of GluN2 subunits of NMDA receptors were reduced, but the level of AMPA receptors remained unchanged. The NMDA receptor currents were also reduced in the forebrain synapses in PSD-93KO animals and the NMDA receptor dependent persistent pain was also reduced (Tao et al., 2003). Genetic knockout of PSD-93 doesn't have any effect on the basal synaptic function in hippocampus but RNAi mediated knockdown showed a 50\% reduction in AMPA currents (Elias et al., 2006a). Over expression of specific isoforms of PSD-93 also showed to increase AMPA receptor mediated currents in hippocampus (Krüger et al., 2013a). 


\subsection{Apoptosis}

Programmed cell death or "apoptosis" was a term coined by Kerr et al (1972) to distinguish a morphologically distinct form of cell death. Apoptosis had been known to occur normally during development and aging as a homeostatic mechanism to maintain the cell population. Apoptosis can be activated by a wide variety of stimuli, coming both from outside or within the cell. Conditions both physiological and pathological can trigger apoptosis but not all cells undergo apoptosis under similar stimulus, thus proving that there is a fine-control in the process of apoptosis.

\subsubsection{Mechanism}

The mechanism of apoptosis is highly complex involving an energy dependent cascade of molecular events. There are two main pathways by which a cell might undergo apoptosis: the extrinsic or the death receptor pathway and the intrinsic or the mitochondrial pathway. However, recent evidence had shown that both these pathways are linked and there are molecules from one pathway that can influence the other pathway as well (Igney and Krammer, 2002). Both the extrinsic and the intrinsic pathway converge on the execution pathway, which is initiated by the cleavage of caspase- 3 and results in DNA fragmentation, degradation of cytoskeletal and nuclear protein, membrane disintegration and finally apoptotic body formation.

The most significant group of enzymes that are required for the process of apoptosis is called caspases. They are a group of serine proteases that are expressed as inactive zymogens which when activated can activate other pro-caspases, thereby initiating a signaling cascade. Due to this sequential activation of one caspase by another caspase, the signal of apoptosis gets amplified and hence the resultant cell death process is rapid and irreversible. Caspases are classified as initiators (Caspase-2, -8, -9, -10), effectors or executioners (Caspase-3, -6, -7) and inflammatory (Caspase-1, -4, -5) (Cohen, 1997; Rai et al., 2005). 


\subsubsection{Extrinsic pathway}

The extrinsic signaling pathway that initiates apoptosis involves trans-membrane receptor mediated interaction. The sequence of events are best characterized with Fas L/Fas R and TNF- $\alpha /$ TNF R1 models. When Fas ligand or TNF- $\alpha$ binds to Fas receptor or TNF Receptor 1 respectively, it results in the binding of adaptor proteins FADD or TRADD respectively (Grimm et al., 1996; Hsu et al., 1995; Wajant, 2002). FADD or TRADD associates with pro-caspase- 8 via dimerization of a death effector domain and activates it auto-catalysis (Kischkel et al., 1995). Active caspase- 8 can activate the effector caspase-3, which in turn completes the process of DNA fragmentation and subsequent cellular mortality.

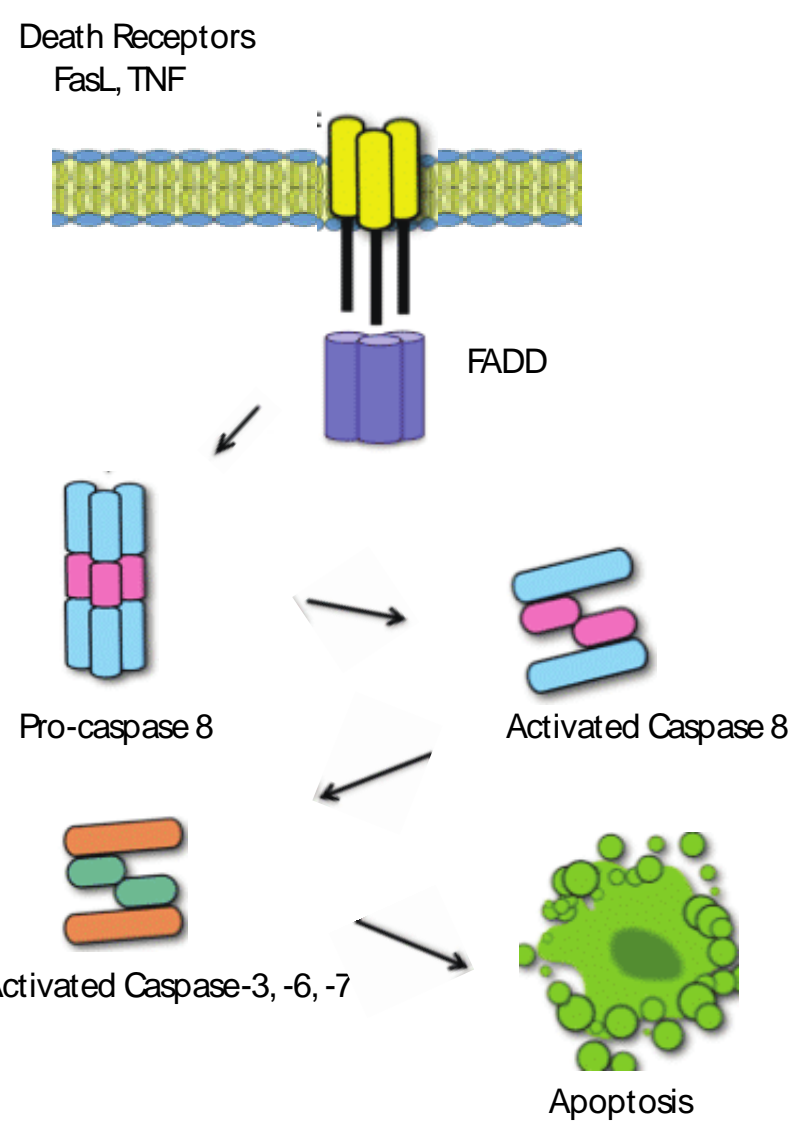

Figure 2: The extrinsic pathway of apoptosis. Members of the death receptor superfamily (FasL, TNF) causes activation of the extrinsic pathway of apoptosis. The binding of FasL to Fas induces receptor clustering and formation of the Death-Inducing Signaling Complex (DISC). This complex can recruit and activate caspase- 8 from its inactive zymogen form. Active caspase- 8 can activate the effector caspase (caspase-3, -6, -7) by direct cleavage and induce apoptosis. (Picture modified from Dawn M., 2012) 


\subsubsection{Intrinsic pathway}

The intrinsic pathway of apoptosis involves a variety of non-receptor mediated stimuli that produce intra-cellular signaling events that act directly on the targets within the cell. These stimuli generate intra-cellular signals that cause change in the inner mitochondrial membrane resulting in an opening of the mitochondrial permeability transition (MPT) pore complex, loss of mitochondrial trans-membrane potential and release of two pro-apoptotic proteins, Cytochrome $\mathrm{C}$ and the serine protease, HtrA2, from the inter-membrane space into the cytoplasm (Saelens et al., 2004). These proteins bind to Apaf-1 and activates the procaspase-9 and forms an apoptosome (Chinnaiyan, 1999; Hill et al., 2004). This then activates the effector caspase-3 and leads the cell towards cell death. The over-activation of NMDARs and subsequent rapid influx of $\mathrm{Ca}^{2+}$ causes activation of two major pathways of apoptosis:

\subsection{MAP kinases pathway of apoptosis}

MAP kinases like P38 and JNK (c-Jun NH(2)-terminal kinase) when activated can activate the transcription factor p53 by phosphorylating it. When activated, p53 induces the expression of pro-apoptotic proteins like Bax and Bad. These proteins can then go and induce permeabilization of the mitochondrial outer membrane causing release of cytochrome $\mathrm{C}$ and the subsequent steps of apoptosis that follows (Chipuk and Green, 2008; Liu et al., 2003).

\subsubsection{2 nNOS pathway of Apoptosis}

Excessive activation of NMDAR during excitotoxic challenges causes excess $\mathrm{Ca} 2+$ influx (Lipton and Rosenberg, 1994). The intracellular $\mathrm{Ca}^{2+}$ triggers the production of nitric oxide by activation of neuronal NO synthase (nNOS) in a $\mathrm{Ca}^{2+/}$ Calmodulin (CaM)-dependent manner (Bredt et al., 1991; Garthwaite et al., 1988). Although, physiologic levels of NO is required for cellular functions, increased accumulation of intense nitrosative stress due to generation of reactive nitrogen species (RNS) can be detrimental to neurons. NO can have two modes of neurotoxic damage. 
a) It can react to cysteine residues of target proteins to form s-nitrosothiols in a process called s-nitrosylation. S-nitrosylation of :

i. Parkin (a ubiquitin E3 ligase) and PDI (protein disulphide isomerase, an endoplasmic reticulum chaperone) causes accumulation of misfolded toxic proteins (Chung et al., 2004; Lipton et al., 2005; Uehara et al., 2006; Yao et al., 2004).

ii. Glyceraldehyde 3-P dehydrogenase (GAPDH) in the cytoplasm transducing in an apoptotic signal into the nucleus of neuronal cells (Hara et al., 2005).

iii. Matrix metalloproteinase (MMP)-2/9, leading to a unique extracellular pathway of excitotoxic cell death (Gu et al., 2002).

b) In addition, it reacts with the reactive oxygen species (ROS) produced by mitochondria, called superoxide anion $\left(\mathrm{O}_{2}^{-}\right)$to form the extremely toxic peroxynitrite radical (ONOO`) (Beckman et al., 1990; Lipton et al., 1993). 
Thus, in general the intrinsic process of apoptosis in neurons can be summarized in Figure 3.

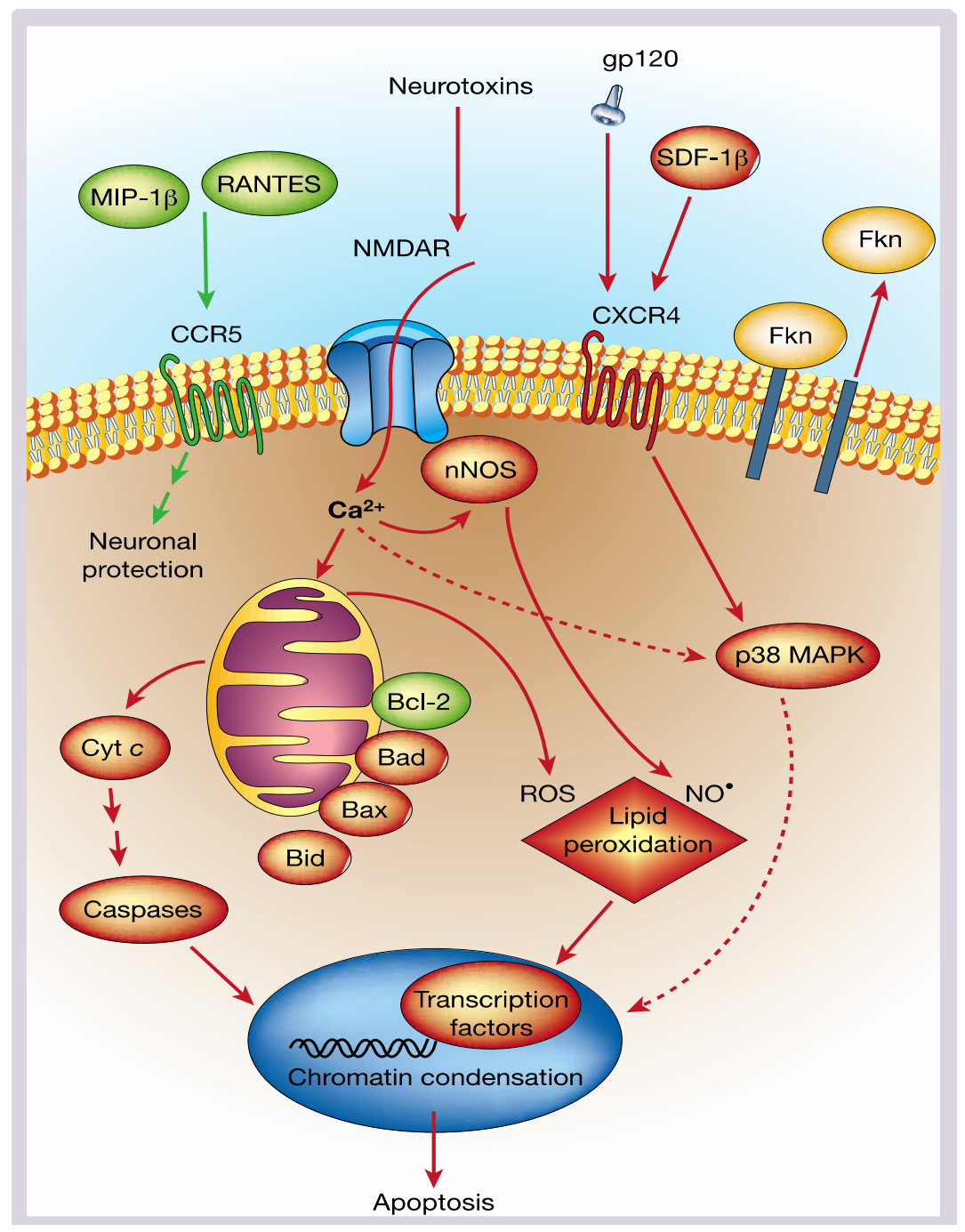

Figure 3: Intrinsic pathways for neuronal apoptosis. Over activation of NMDARs and consequent influx of $\mathrm{Ca} 2+$ into neurons triggers activation of P38 MAPK, mitochondrial $\mathrm{Ca} 2+$ overload and release of cytochrome $\mathrm{c}$ (Cyt c), free radical generation (nitric oxide $(\mathrm{NO}+$ ) and reactive oxygen species (ROS)), caspase activation and apoptosis. NMDARs are physically tethered to neuronal nitric oxide synthase (nNOS), facilitating its activation. The Bcl-2 family members Bad, Bax and Bid promote apoptosis mediated by glutamate, ROS and TNF- $\square$, respectively. Bcl-2 prevents apoptosis, apparently by attenuating cytochrome c release and ROS production. Activation of the p38 MAPK pathway by a Ca2+-mediated mechanism and possibly by oxidative stress may lead to phosphorylation/activation of transcription factors involved in apoptosis (Kaul et al., 2001). 


\subsection{NMDA Receptors: balancing neuro-protection and neuro- toxicity}

NMDA receptors are known to play a very critical role in synaptic plasticity (Barria and Malinow, 2002; Carroll et al., 1999), synaptic development (Constantine-paton et al., 1990; Kerchner and Nicoll, 2008) and glutamate induced neurotoxicity (Choi, 1988a; Tu et al., 2010a). Studies have found that NMDA receptor functions have been severely perturbed in a variety of CNS diseases ranging between traumatic brain injury, neuro-degeneration and ischemic stroke (Koutsilieri and Riederer, 2007; Liu et al., 2007). Many suspect that glutamate induced neurotoxicity could be the underlying key-mechanism behind many of these CNS injuries. The mechanisms of glutamate induced neurotoxicity happens mostly through the NMDA receptors, owing to their high permeability to $\mathrm{Ca}^{2+}$, as opposed to AMPA receptors which are mostly $\mathrm{Ca}^{2+} \mathrm{im}-$ permeable (Tu et al., 2010a; Wenthold et al., 1996). The overly released glutamate from the presynaptic terminal during CNS injuries may cause excess $\mathrm{Ca}^{2+}$ loading via the NMDA receptors in the post-synaptic compartment and activate neuronal mortality genes (Benveniste et al., 1984; Okamoto et al., 2009).

Pharmaceutical intervention approaches using NMDA receptor antagonists had been largely met with disappointing results at clinical trial levels (Kemp and McKernan, 2002; Traynor et al., 2006). This is due to the fact that the different subunits of NMDA receptors, GluN2A and GluN2B, activate different intra-cellular signaling cascades upon activation. While the GluN2A activation has been shown to be pro-cell survival, the activation of GluN2B has been shown to be pro-apoptotic. Hence, a nonspecific blockade of NMDA receptor may block not only cell mortality but as well, the cell survival pathways in neurons. Thus targeting specific intra-cellular pathways downstream of GluN2A or GluN2B receptors may provide a better mechanistic control over the pathway we want to block during CNS injuries. 


\subsubsection{GluN2A receptors in neuro-protection}

NMDA receptors with the GluN2A subunit are known to have a protective function and responsible for cell survival during CNS injuries (Anastasio et al., 2009; Chen et al., 2008; DeRidder et al., 2006). NMDA receptor that contains the GluN2A subunits, are known to preferentially localize in the synaptic zone (Stocca and Vicini, 1998; Tovar and Westbrook, 1999). It is now predicted that location of a receptor, either synaptic or extra-synaptic, is a primary determinant of the down stream function that it has. In hippocampal neurons for example, the activation of GluN2A causes a preferential activation of CREB, which in turn activates BDNF and cpg 15 gene expression and promotes cell survival (Chen et al., 2008; Hardingham et al., 2002). Again, Liu et al., 2007 found that in rat cortical neurons GluN2A activation from both synaptic as well as extra-synaptic pool, promotes cell survival and it does so by activation of the Akt-dependent pathway.

In support of this existing hypothesis, experiments have shown that pharmacologic blocking of GluN2A leads increased cellular mortality during transient global ischemia in rats and causes potentiation of caspase-3 (Chen et al., 2008; DeRidder et al., 2006).

\subsubsection{Signaling pathway}

The principle pathway, through which GluN2A imparts its neuro-protection during CNS injuries, is the GluN2A-PTEN-TDP-43 pathway (Zheng et al., 2012). GluN2A activation causes the reduction in expression level of PTEN (aka, Phosphatase and Tensin Homolog). PTEN is known to play a role in neuronal injuries such as ischemia and neurodegenerative diseases (Gary and Mattson, 2002; Omori et al., 2002). Suppression of PTEN is known to increase cell survival (Cantley and Neel, 1999; Chang et al., 2007; Ning et al., 2004) by increasing the level of another nuclear pro-survival marker called TDP-43 (TAR DNA-binding protein-43). How TDP-43 helps cell survival is still vaguely understood. Nuclear knock down of TDP-43 is detrimental to neurons and promotes neuro degenerative signaling (Fiesel et al., 2010; Iguchi et al., 2009; Zheng et al., 2012). So activation of GluN2A down-regulates PTEN, which in 
turn up-regulates TDP-43 and increases neuronal cell survival. Interestingly GluN2B has no effect on PTEN expression.

\subsubsection{GluN2B in neuronal death and neuro-degeneration}

GluN2B in recent years had been credited mainly for the neuro-degenerative role that NMDA receptors play during CNS injuries. Recent findings claim that GluN2B activation is involved during neurotoxic insults (Liu et al., 2007; Tu et al., 2010a). However complete blockade of GluN2B receptors using pharmacologic antagonist haven't been successful as they also interfere with the channels principle physiological functions (Rodrigues et al., 2001; Walker and Davis, 2008; Zhao et al., 2005).

\subsubsection{Signaling pathway}

The activation of the apoptotic pathway via the Map Kinase P38 has long been associated with glutamate excito-toxicity (Barone et al., 2001). Also, the role of NMDA receptor in activation of P38 has been proved (Kinases et al., 1999; Waxman and Lynch, 2005a). However the precise mechanism that regulates the duration and magnitude of P38 activation is not completely understood. A key molecule that regulates the mechanism of P38 MAPK activation is the Striatal-enriched Tyrosine Phosphate or STEP, also known as PTPN5. This protein is known to be expressed specifically in the striatum, neo-cortex and hippocampus (Boulanger et al., 1995). Both STEP 61 and STEP $_{46}$, the two isoforms of the protein (Bult et al., 1997) contain a highly conserved substrate-binding motif called the Kinase Interacting Motif (KIM) domain (Pulido et al., 1998). Phosphorylation of the Ser221 within this KIM domain renders the STEP enzyme inactive. This phosphorylation is mediated by a dopamine/D1 receptor mediated activation of Protein Kinase A (aka, PKA) (Paul et al., 2000).

Now when GluN2B subunit is activated for a brief time, it causes influx of $\mathrm{Ca}^{2+}$ ions. This causes de-phosphorylation of STEP by a $\mathrm{Ca}^{2+}$ dependent phosphatase calcineurin (Paul et al., 2003). Active STEP can contribute to neuronal survival by dephosphorylating and thus inactivating P38 MAPK. 
But upon sustained GluN2B activation, another $\mathrm{Ca}^{2+}$-dependent protease called calpain gets activated which can cleave STEP $_{61}$ and can initiate a secondary activation of P38 (Xu et al., 2009). There are two kinds of calpain, the $\mu$-calpain (calpain-1) which is more synaptic and the m-calpain (calpain-2), which is more extra-synaptic. In recent years m-calpain activation had been linked to a wide range of pathological status that includes stroke, neuro-degeneration and traumatic nerve injury (Liu et al., 2008; Vosler et al., 2008; Xu et al., 2009). It is believed that upon sustained activation of extra-synaptic GluN2B receptors, the m-calpain gets activated. This in turn cleaves the active $\mathrm{STEP}_{61}$ in its KIM domain, thus activating the extra-synaptically concentrated pool of P38.

\subsection{NMDA receptors, DLG-MAGUKs and Ischemia}

It was established so far that, NMDA receptors in addition to their roles in synaptic transmission have a very important role to play in ischemic brain injuries (Simon et al., 1984). But blocking the NMDA receptors have yielded no fruitful results, as it also blocked the synaptic transmissions of these receptors and caused detrimental effects in animals and humans (Aarts et al., 2002; Davis et al., 2000; Fix et al., 1993). Another alternative strategy was to block the down stream signaling pathways that connects NMDA receptors with the excito-toxic pathway without tampering with the synaptic transmission properties.

PSD-95, the post-synaptic signaling scaffold molecule was known to couple NMDA receptors with intra-cellular signaling cascades (Brenman et al., 1996; Sheng, 2001). Using anti-sense PSD-95 oligo nucleotide in cortical neurons, that targets the PSD-95NMDAR interaction, neuronal mortality against NMDAR mediated neurotoxicity could be blocked (Sattler, 1999a). Since, suppression of PSD-95 is an impractical therapeutic strategy, owing to its very important role in maintaining post-synaptic transmission, a finer approach was adopted by targeting the PSD-95-GluN2B interaction. This disruption was achieved by using an inhibitory peptide containing nine Cterminal residues of GluN2B (KLSSIESDV; GluN2B9C) (Kornau et al., 1995a). This peptide was made cell permeable by fusing it with the cell-membrane transduction 
domain of Human Immunodeficiency Virus-1 Tat protein (YGRKKRRQRRR; Tat) (Schwarze, 1999) to obtain the twenty amino acid peptide Tat-GluN2B9C. Administration of this peptide could decrease the neurotoxic vulnerability of cortical neurons to the same level as was observed in the PSD-95 knockdown cells. In addition it made neurons resistant to focal cerebral ischemia in vivo (Aarts et al., 2002).

However the human genome has reported hundreds of different PDZ-domain containing proteins (Giallourakis et al., 2006) and each of the NR subunit C-terminal contains a promiscuous PDZ interaction motif (T/SXV). Consequently the NR subunits might bind to other PDZ-domain containing proteins and mediate its neurotoxic properties independent of PSD-95. Alternatively, the Tat-GluN2B9C may also cause either its neuro-protection or additional side effects by perturbing the NR subunit-PDZ interaction with a target other than PSD-95.

\subsection{Scope of the Thesis}

It is still unclear if PSD-93 has any essential role in maintaining surface level of glutamate receptors and if this regulation is brain region specific. The first aim of the study was to identify if PSD-93 regulates the surface level of AMPA and NMDA receptors at excitatory synapse in mice visual cortex. There are various mechanisms by which receptor levels are maintained at neuronal membrane, including phosphorylation of specific residues on these receptors. I would like to check the mechanism by which PSD-93 might regulate the surface level of these receptors. Since NMDA receptors are also crucially linked with glutamate induced neurotoxicity during ischemic brain injuries, the second aim was to check the functional relevance that PSD-93 has in such forms of neurotoxicity and to identify which isoform(s) of PSD-93 is particularly responsible for it. I also wanted to identify the molecular signaling pathway(s) that may be required by PSD-93 for linking glutamate receptor over-activation with apoptotic signaling during excitotoxicity. PSD-95 had been long identified as a link between NMDA receptor over-activation and neuronal apoptosis. I wanted to compare, between PSD-95 and PSD-93, who has a more potent role in mediating the 
NMDA receptor based neurotoxicity in cortical synapses and if they have the same or have different mode of operation during neurotoxic challenges. 


\section{Materials and Methods}

\subsection{Molecular Biology}

\subsubsection{Cloning}

Standard methods were followed for cloning (Sambrook, 2001). The chemicals and kits used for cloning were obtained from Bioline (Luckenwalde, Germany), Fermentas/Thermofischer Scientific (Waltham, MA, USA), NEB (Frankfurt, Germany) and Qiagen (Hilden, Germany). 


\subsubsection{Polymerase Chain Reaction}

For amplification of specific DNA segments, the standard PCR method was employed (Saiki et al., 1985). The Table 1 below indicates the amount of the reagents used for the reactions:

Table 1: PCR Reaction

\begin{tabular}{ll}
$\boldsymbol{P C} \boldsymbol{R}$ Mix & \\
\hline Template DNA & $20 \mathrm{ng}$ \\
10x Reaction Buffer (Bioline/Fermentas) & $2 \mu \mathrm{l}$ \\
dNTP's (2.5 mM each, Bioline) & $2 \mu 1$ \\
$50 \mu \mathrm{M}$ Forward primer & $0.5 \mu 1$ \\
$50 \mu \mathrm{M}$ Reverse primer & $0.5 \mu 1$ \\
Taq*/Pfu**Polymeras(Bioline/Fermentas) & $1 \mu 1$ \\
\cline { 2 - 2 } & $20 \mu 1$
\end{tabular}

PCR products were run on $1 \%$ agarose gel prepared in TAE buffer $(0.2 \% 0.5 \mathrm{M}$ EDTA, $1.14 \mathrm{ml} / \mathrm{l}$ Acetic acid, $30 \mathrm{mM}$ Tris) to confirm and purify PCR products. 


\subsubsection{Restriction Digestion}

For confirming DNA constructs, and also preparing DNA fragments for cloning into vectors, restriction digestion was performed using restriction enzymes from Fermentas and NEB in their respective buffer systems. The typical digestion reaction has been shown in table

Table 2: Restriction Digestion Reaction

\section{Restriction Digestion Reaction Mix}

\begin{tabular}{ll} 
DNA sample & $1 \mu \mathrm{l}(1.5 \mu \mathrm{g})$ \\
10X reaction buffer & $5 \mu \mathrm{l}$ \\
BSA & $0.5 \mu \mathrm{l}$ \\
Restriction Enzyme & $2 \mu \mathrm{l}$ \\
$\mathrm{H}_{2} \mathrm{O}$ & $41.5 \mu \mathrm{l}$ \\
\hline & $\frac{50 \mathrm{l}}{}$
\end{tabular}

The reaction was incubated at $37^{\circ} \mathrm{C}$, unless otherwise specified by the enzyme used, for $2 \mathrm{~h}$. The digested sample was mixed with 1x TAE gel loading buffer and run on $1 \%$ agarose gel. The band of the desired size was visualized and excised under a UV trans-illuminator. The excised product was further purified as mentioned in the next section. 


\subsubsection{PCR product and agarose gel purification}

The PCR product and restriction digested vectors were isolated according to the manufacturer's protocol from Qiagen (Hilden, Germany). The isolated fragments were eluted out in $30 \mu \mathrm{l}$ of 1:10 diluted elution buffer (Qiagen). The isolated fragments were used in subsequent steps of ligation.

\subsubsection{Ligation}

For the cloning protocol, inserts were ligated to different vector backbones in a molar ratio of $1: 3$. The vector was heated at $50^{\circ} \mathrm{C}$ for $5 \mathrm{~min}$ and snap chilled right before the reaction to avoid con-catamerization . The reaction was set as follows:

Table 3: Ligation Reaction

\section{Ligation Reaction Mix}

Vector

$1.5 \mathrm{ng}$

Insert

$0.5 \mathrm{ng}$

T4 DNA ligase buffer (Fermentas) $\quad 3 \mu \mathrm{l}$

T4 DNA Ligase (Fermentas) $\quad 1 \mu \mathrm{l}$

$\overline{30 \mu 1}$

The ligation was carried out at $16^{\circ} \mathrm{C}$ overnight. The ligation products were precipitated using $125 \mathrm{mM} \mathrm{NaCl}$ and twice volume of absolute ethanol. The precipitate was finally resuspended in $10 \mu \mathrm{ls}$ of deionized water and $1 \mu \mathrm{l}$ of this was used for transformation. 


\subsubsection{Transformation}

$1 \mu \mathrm{l}$ of the ligation reaction was transformed into $50 \mu \mathrm{l}$ of electro-competent XL1Blue Escherichia coli (E. coli) strain (Stratagene, Santa Clara, CA, USA) and colonies were cultivated on LB-agar plates containing ampicillin (100 $\mu \mathrm{g} / \mathrm{ml}$, Roth)

\subsubsection{Plasmid Preparation}

For amplification of the ligated plasmids, single colony was picked up from the transformed bacterial plate and inoculated in either $5 \mathrm{ml}$ (mini-prep) or $50 \mathrm{ml}$ (midi-prep) of LB medium containing $100 \mu \mathrm{g} / \mathrm{ml}$ of ampicillin to select for only transformed cells. The cultures were allowed to grow overnight at $37^{\circ} \mathrm{C}$ under vigorous shaking.

The bacteria was precipitated by centrifugation at $7000 \mathrm{~g}$ for $10 \mathrm{~min}$ and the plasmid extraction was achieved following Qiagen (Hilden, Germany) DNA isolation protocol followed by alkaline lysis (Birnboim and Doly, 1979). To get rid of the RNAs, the samples were treated with RNase at $55^{\circ} \mathrm{C}$ for $5 \mathrm{~min}$. To purify the plasmid from all protein contamination, phenol-chloroform extraction was performed. To the plasmid extract, equal volumes of phenol:chloroform:isoamyl alcohol (25:24:1) (Applichem) was mixed and applied on a phase lock tube (Eppendorf).The clear aqueous phase isolated after centrifugation and mixed with an equal volume of chloroform and centrifuged again. The aqueous phase was separated and the DNA was precipitated with 0.7 volume of isopropanol and the pellet dissolved in $30 \mu \mathrm{l}$ of elution buffer (Qiagen) for mini-prep and 300-400 $\mu 1$ for midi-prep.

$1 \mu \mathrm{l}$ of the plasmid was run on $1 \%$ agarose gel in TAE buffer to confirm the concentration. The sequencing of the constructs were performed and analyzed at AGCT Sequencing Facility of MPI-EM (Göttingen). 


\subsubsection{Genotyping}

PSD-93KO mouse line is described by McGee et al (2001b) and was kept in the institutes animal facility. For genotyping of the PSD-93 animals the protocol described by The Jackson Laboratory (Bar Harbor, ME, USA) was followed: $2 \mathrm{~mm}$ tail samples were used for the genotyping reactions at age P0 for knockout culture preparation and age P4-P6 for biochemical experiments with P20-P30 mice. Tail samples were mixed with 100-200 $\mu \mathrm{l}$ of PBND lysis buffer (10 mM Tris, $50 \mathrm{mM} \mathrm{KCl,} 2.5 \mathrm{mM}$ $\mathrm{MgCl}_{2} * 6 \mathrm{H} 2 \mathrm{O}, 0.1 \mathrm{mg} / \mathrm{ml}$ Gelatine, $0.45 \%$ (v/v) Nonident P40, 0.45\% (v/v) Tween $20, \mathrm{pH}=8,3$ ) containing $1.2 \mathrm{mg} / \mathrm{ml}$ Proteinase $\mathrm{K}$ (Roth) at $55^{\circ} \mathrm{C}$ under $1000 \mathrm{rpm}$ agitation or three hours under $1400 \mathrm{rpm}$ agitation. Samples were then heated up to $99^{\circ} \mathrm{C}$ for $10 \mathrm{~min}$ in order to inactivate the proteinase $\mathrm{K}$. The sample tubes were further centrifuged on a tabletop centrifuge at highest speed for 5 min to separate the debris and $2 \mu \mathrm{l}$ of the supernatant was used for subsequent PCR reactions (Table 5). The wildtype allele showed a band corresponding to a molecular weight of $330 \mathrm{bp}$ and the knockout allele showed a band of size $750 \mathrm{bp}$.

Table 4: Primers used for wild-type and PSD-93 knockout animal genotyping.
PCR primers for genotyping
Sequences

p93-intron

GTGCGGAATGTTGTTGTGCAGTGC

p93-exon'

CACAACAGTCTCCAATATGGGTCGC

p93-neo'

GCCTTCTATCGACTTCTTGACGAG 
Table 5: PCR mix used for wild-type and PSD-93 knockout animal genotyping

\section{PCR Mix}

Sample

$2 \mu \mathrm{l}$

10X TNK Buffer

$2.2 \mu 1$

dNTP's (2.5mM, Bioline)

$2 \mu \mathrm{l}$

Primer 1

$0.2 \mu 1$

Primer 2

$0.2 \mu 1$

Primer 3

$0.2 \mu 1$

$\mathrm{H}_{2} \mathrm{O}$

$15 \mu 1$

Mango Taq (polymerase)

$0.2 \mu 1$

$22 \mu 1$ 
Table 6: PCR program for wild-type and PSD-93 knockout animal genotyping.

\section{PCR Program}

Time [min] Temperature $\left[{ }^{\circ} \mathrm{C}\right]$

Pre-incubation

2

94

Denaturation

15

94

Annealing

$45^{\prime}$

57

x35

Elongation

1

72

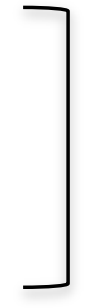

Final Elongation

4

72

\subsubsection{Constructs used during study}

For over-expression analysis of different N-terminal isoforms of PSD-93, bi-cistronic lentiviral constructs containing the N-terminus of different PSD-93 isoforms and the rest from rat PSD-93 sequence were used (named as FUp93xGW ( $\mathrm{x}=\alpha_{1 / 2}, \beta, \gamma, \delta$, and ع) (Krüger et al., 2013a). For the knockdown of PSD-95, the lentiviral vector based constructs FRUGW, which contains RNAi against endogenous PSD-95 was used (Schlüter et al., 2006). 


\subsection{Cell Culture}

The chemicals used for performing cell culture was primarily ordered from Biochrom (Berlin, Germany), Sigma Aldrich (München, Germany), Millipore (Schwalbach, Germany), Roth (Karlsruhe, Germany), Merck (Darmstadt, Germany), Greiner Bioone (Frickenhausen, Germany), Invitrogen/Life Technologies (Darmstadt, Germany), Thermofischer Scientific (Waltham, MA, USA) and Roche (Mannheim, Germany).

\subsubsection{HEK293T cell line}

Human embryonic kidney, HEK293T was used for the preparation of small and large scale virus production. Cells were cultured in $10 \mathrm{~cm}$ cell culture dishes in presence of High glucose Dulbeccos's Modified Eagle's Medium (Biochrom) containing 10\% fetal calf serum (Biochrom). Cells were incubated at $37^{\circ} \mathrm{C}$ for $2-3$ days till they reached a confluency of $75-80 \%$ when they were passaged at a dilution of 1:15- 1:20.

\subsubsection{Lentivirus production}

Lentivirus particles for gene transfection were carried out in HEK293 cell line using HIV-1 packaging vector 8.9 and envelope glycoprotein vector Vsvg along with the plasmid of interest (Lewis et al., 1992). For small-scale production of virus particle from HEK293 cells, $6 \mathrm{~cm}$ dishes and for large scale production, T-75 tissue culture flasks were used. The cultures were infected at $60-70 \%$ confluency with the amount of the various DNA constructs given in the table below: 
Table 7: DNA mix for lentivirus production.

DNA construct

Plasmid

$\Delta 8.9$ vector

Vsvg vector
Small Scale production

$2 \mu \mathrm{g}$

$1.5 \mu \mathrm{g}$

$0.5 \mu \mathrm{g}$
Large Scale production

$10 \mu \mathrm{g}$

$7.5 \mu \mathrm{g}$

$2.5 \mu \mathrm{g}$

One day after the infection, the transfected plates were transferred to $32^{\circ} \mathrm{C}$ incubator with $5 \%$ equilibrated $\mathrm{CO}_{2}$ concentration in order to minimize cell mortality and increase viral production.

For small-scale virus production, the wells from 6 wells plates were harvested 2 days post transfection. The supernatant media was carefully collected and centrifuged at 2000xg for 5 min to isolate the viral particles from the cell debris. The supernatant from this centrifugation, which contained the virus were isolated, aliquoted in $1.5 \mathrm{ml}$ tubes and stored at $-80^{\circ} \mathrm{C}$ freezer for further usage.

For large-scale virus production, the supernatant of the T-75 flask was harvested and centrifuged at $2000 \mathrm{xg}$ for $5 \mathrm{~min}$ to separate the cell debris. This fraction is further passed through PVDF membrane with $0.45 \mu \mathrm{m}$ pore size (Millipore) and viral particles precipitated in an ultra-centrifuge at $36,500 \mathrm{~g}$ for $1.5 \mathrm{~h}$. The pellet containing the viral particles were left overnight to re-suspend in virus storage buffer (20 mM Tris$\mathrm{HCl}(\mathrm{pH}=8.0), 250 \mathrm{mM} \mathrm{NaCl}, 5 \%$ Sorbitol $)$ and aliquoted and stored in $-80^{\circ} \mathrm{C}$ for further usage. 


\subsubsection{Dissociated hippocampal culture}

Dissociated hippocampal cultures were prepared from P0 or P1 mice or rat hippocampi. The animals were anesthetized on ice for 2-4 min and then were decapitated. The brain was carefully taken out and placed in dissection solution (mGBSS) solution. The two hemispheres of the brain were separated using micro-dissection forceps. The meninges were carefully removed and an incision was made with a sharp microscalpel on both ends of the hippocampus. Next, The hippocampus was folded out of the cortex and detached. The dentate gyrus was removed using a pair of forceps. The isolated hippocampi were transferred first to a fresh dissection solution and then to Enzymatic Solution (ES: 11.39 mM L-cysteine, $50 \mathrm{mM}$ Na-EDTA pH=8, $10 \mathrm{ml}$ DS, $10 \mathrm{mM} \mathrm{CaCl}_{2}, 1 \mathrm{~N} \mathrm{HCl}, 100 \mu 10 \mathrm{mg} / \mathrm{ml}$ DNase I, $100 \mu \mathrm{l}$ Papain) and put on a mild rotation at $37^{\circ} \mathrm{C}$ for $30 \mathrm{~min}$. After digestion the solution was replaced with Inactivation Solution (IS: $10 \mathrm{ml}$ 5\% Serum Medium (25 ml Fetal Bovine Serum, $500 \mathrm{ml}$ MEM without Earle's salts and L-glutamine, $7.5 \mathrm{ml} \mathrm{Hi-Glucose} \mathrm{MEM,} 1 \mathrm{ml} \mathrm{Mito+}$ Serum Extender), $100 \mu 110 \mathrm{mg} / \mathrm{ml}$ DNase I, $25 \mathrm{mg}$ BSA) and the hippocampi were let to settle for about $2 \mathrm{~min}$. The Inactivation Solution was next removed and replaced with one hub of $5 \%$ Serum media(25 ml Fetal Bovine Serum, $500 \mathrm{ml}$ MEM without Earle's salts and L-glutamine, 7.5 ml Hi-Glucose MEM, $1 \mathrm{ml}$ Mito+ Serum Extender). Using a $2 \mathrm{ml}$ fire-polished glass pipette, the hippocampi were triturated very gently couple of times and allowed to sit for another $2 \mathrm{~min}$. The supernatant was collected in a fresh $10 \mathrm{ml}$ tube and the undigested chunks were resuspended again with one hub of $5 \%$ serum media and the procedure repeated. The supernatant was centrifuged at $500 \mathrm{~g}$ for $5 \mathrm{~min}$ and the supernatant was discarded. The pellet was resuspended in $1 \mathrm{ml}$ of $5 \%$ serum media and cells were counted in a Neubauer chamber and 100.000 cells were plated onto each well of Poly-D-lysine (PDL) pre- coated 24 well plate.

The cultures were treated with FUDR (Sigma Aldrich) on DIV4 to restrict the further growth of astrocytes and were fed on DIV7 by exchanging half of the media with fresh media without Glutamate and Aspartate. Feeding was repeated every 3-5 days as was required. 


\subsubsection{Lentiviral infection of dissociated neuronal culture}

For transfection of dissociated culture, lenti virus carrying the plasmid of interest was used on DIV7. For each well of a 24 well plate about $100 \mu \mathrm{l}$ of the small scale or $1 \mu \mathrm{l}$ of the large-scale virus was used. Cultures were monitored for the fluorescence after DIV 12 and harvested between DIV 14-18 as was required for the experiment 


\subsection{Biochemistry}

\subsubsection{Western Blotting sample preparation from dissociated neuronal culture}

For western blot analysis of dissociated neuronal cultures from mice or rat, primary cultures at DIV 14 or later, as per the requirement of the experiment, was washed with ice cold PBS with Mg and Ca. The cells were scraped out and harvested in 2x SDS sample buffer (3.3\% Tris- $\mathrm{HCl}, 3.4 \%$ Tris, 8\% SDS, 0.02\% Sucrose, 0.03\% EDTA, $0.02 \%$ bromophenol blue, $0.27 \%$ DTT (w/v)). For each well of a 24-well plate, $40 \mu 1$ of 2X SDS Sample buffer and for each well of a 6 well plate, $200 \mu$ ls of the same was used. Samples were heated up to $55^{\circ} \mathrm{C}$ for $15 \mathrm{~min}$ with $1000 \mathrm{rpm}$ agitation or at $95^{\circ} \mathrm{C}$ for 5 min without agitations. Samples were given a short centrifugation prior to storing them at $-20^{\circ} \mathrm{C}$ until further usage.

\subsubsection{Co-Immunoprecipiation Assay}

In order to verify direct physical interaction between specific proteins in the cellular milieu, co-immunoprecipiation assays were performed. Primary mice cortical cultures in 6 well plates were washed in ice-cold PBS with $\mathrm{Mg}^{2+} / \mathrm{Ca}^{2+}$. The cells were then harvested in RIPA lysis buffer (50 mM NaH2PO4, $150 \mathrm{mM} \mathrm{NaCl}, 1 \%$ Triton X-100, $0.1 \%$ SDS, $0.5 \%$ deoxycholic acid, $2 \mathrm{mM}$ EDTA and Roche protease inhibitor cocktail, $\mathrm{pH}=7.4$ ) and set on a rotator for $1 \mathrm{~h}$ at $4^{\circ} \mathrm{C}$. After $1 \mathrm{~h}$, the lysate was centrifuged at $15,000 \mathrm{xg}$ for $30 \mathrm{~min}$ at $4^{\circ} \mathrm{C}$. The clean supernatant was collected and was prewashed for $1 \mathrm{hr}$ at $4{ }^{\circ} \mathrm{C}$ with protein $\mathrm{A} / \mathrm{G}$ conjugated beads, that had already been washed and equilibrated in washing buffer (10 mM Tris/HCl, $1 \mathrm{mM}$ EDTA, $250 \mathrm{mM}$ $\mathrm{NaCl}, 0.5 \% \mathrm{TX}-100, \mathrm{pH}=7.4$ ), to avoid any non-specific interaction in the subsequent stages of the protocol. The supernatant was centrifuged at $7000 \mathrm{rpm}$ for $15 \mathrm{~min}$ 
at $4{ }^{\circ} \mathrm{C}$. To the supernatant was added $1 \mu \mathrm{g}$ of antibody to pull down the primary protein of interest. The binding was established by an over-night incubation at $4{ }^{\circ} \mathrm{C}$ with mild rocking. The next day, a small aliquot of this tube was collected and kept on ice as the input sample for further processing. To the rest of the tube, protein conjugated beads were added and incubated for another $2 \mathrm{~h}$ at $4{ }^{\circ} \mathrm{C}$ with mild rocking. The antibody and bead bound proteins were pulled down by applying a centrifugation of 7000 $\mathrm{rpm}$ at $4{ }^{\circ} \mathrm{C}$ for $15 \mathrm{~min}$. The precipitate was washed in washing buffer for three times of 5 min each and lastly once with 1X TBS (pH7.4). Lastly, 2X SDS sample buffer was added to the bead bound proteins as well as the input sample and heated at $95^{\circ} \mathrm{C}$ for $10 \mathrm{~min}$, centrifuged at $14,000 \mathrm{xg}$ for 1 minute and stored at $-20^{\circ} \mathrm{C}$ until further use.

\subsubsection{Sub-cellular fractionation}

Isolation of crude synaptosomal and/or PSD fractions were done from mice or rat cortical cultures. Cells were washed from a 6 well plate with ice-cold PBS with $\mathrm{Mg}^{2+} / \mathrm{Ca}^{2+}$ and then scraped out in cold homogenization buffer $(10 \mathrm{ml} /$ one mouse cortex, $320 \mathrm{mM}$ sucrose, $10 \mathrm{mM}$ HEPES/NaOH, pH 7.4) (Cho et al., 1992; Schlüter et al., 1999). The cell suspension was homogenized using 10-12 strokes in a Balch homogenizer with a pore size of $10 \mu$. The homogenate was next centrifuged at $1000 \mathrm{xg}$ at $4^{\circ} \mathrm{C}$ for $15 \mathrm{~min}$. The supernatant (S1) was isolated and the pellet (P1) containing the cell debris discarded. The S1 supernatant was centrifuged at $10,000 \times \mathrm{xg}$ at $4{ }^{\circ} \mathrm{C}$ for $15 \mathrm{~min}$ to isolate the crude synaptosomal fraction (P2). The pellet was resuspended in the re-suspension buffer (50 mM NaF, $50 \mathrm{mM}$ HEPES/NaOH, pH 7.4, $1 \mathrm{mM}$ EDTA, $0.2 \mathrm{mM} \mathrm{NaVO} 4,100 \mathrm{mM}$ KCL, protease inhibitor cocktail (Roche)) for a final protein concentration of $1 \mu \mathrm{g} / \mu \mathrm{l}$ approximately. Resuspended samples were treated with $1 \%$ Triton X-100 for $15 \mathrm{~min}$ on ice and centrifuged at 32,000xg for $1.5 \mathrm{~h}$ in an ultracentrifuge. The supernatant formed the Triton soluble fraction (1TS) and the pellet was the Triton Insoluble PSD enriched fraction (1TP). The 1TP pellet was resuspended in re-suspension buffer and protein concentrations of fractions measured using a BCA kit (Pierce). 2X SDS sample buffer was finally added and samples boiled at $55^{\circ} \mathrm{C}$ for $15 \mathrm{~min}$ and stored at $-20^{\circ} \mathrm{C}$ until further use. 


\subsubsection{Western Blotting}

For the biochemical analysis of proteins isolated either from animal tissues, primary neuronal cultures or HEK cells, were run on a denaturing Bis-tris PAGE (Graham et al., 2005; Shapiro et al., 1967) followed by wet wetern blotting (Towbin et al., 1979) onto nitrocellulose or PVDF membranes. The gels for electrophoresis were prepared following the given recipe:

Table 8: Recipies for Bis-Tris gels used for Western blotting.

$10 \%$ Resolving Gel (375 mM Bis-Tris, $\mathrm{pH}=6.2$ - 6.4)

$\mathrm{H}_{2} \mathrm{O} \quad 7.3 \mathrm{ml}$

AMBA $\quad 6.7 \mathrm{ml}$

3.5X Bis-Tris Buffer $\quad 6.0 \mathrm{ml}$

$10 \%$ SDS $\quad 100 \mu 1$

$10 \%$ APS $\quad 120 \mu 1$

TEMED $25 \mu \mathrm{l}$ 


\begin{tabular}{ll}
\hline $\mathrm{H}_{2} \mathrm{O}$ & $6.2 \mathrm{ml}$ \\
AMBA & $1 \mathrm{ml}$ \\
$3.5 \mathrm{X}$ Bis-Tris Buffer & $0.8 \mathrm{ml}$ \\
$10 \%$ SDS & $100 \mu \mathrm{l}$ \\
$10 \%$ APS & $96 \mu \mathrm{l}$ \\
TEMED & $20 \mu \mathrm{l}$ \\
\hline
\end{tabular}

Approximately 10-30 $\mu \mathrm{gs}$ of proteins were run on the SDS-PAGE at $140 \mathrm{~V}$ for $2 \mathrm{~h}$ or more until a good separation of the desired protein was achieved. The gel was next transferred onto a nitro-cellulose membrane in transfer buffer $(25 \mathrm{mM}$ Tris, $191 \mathrm{mM}$ Glycin, 20\% Methanol) at $250 \mathrm{~mA}$ for two hours at $4^{\circ} \mathrm{C}$. The success of the preotein transfer was confirmed by staining the membrane with Ponceau S, a non-specific protein dye. The Ponceau S was washed off using TBS-T(20 mM Tris/HCl, $140 \mathrm{mM}$ $\mathrm{NaCl}, 0.1 \%$ Tween 20, $\mathrm{pH}=7.6$ ) and blocked with 5\% BSA in TBS-T for $30 \mathrm{~min}$ at room temperature on a rocker. The membrane was washed with TBS-T for three times 10 min each. Primary antibody solutions were prepared in TBS-T containing $2 \% \mathrm{BSA}$ and $0.1 \% \mathrm{NaN}_{3}$. Primary antibody incubations were performed for $2 \mathrm{~h}$ at room temperature or over-night at $4^{\circ} \mathrm{C}$, with mild shaking. The membranes were washed for three times 10 min each with TBS-T and secondary antibody binding was performed in TBS-T for $2 \mathrm{~h}$ at room temperature in a light protected chamber.

Afterwards, the membrane was washed with TBS-T three times $10 \mathrm{~min}$ each and a final wash in TBS (20 mM Tris/HCl, $140 \mathrm{mM} \mathrm{NaCl}, \mathrm{pH}=7.6$ ) was performed for 5 min at the very end. Visualization and analysis of band intensity was performed by using the Odyssey Infrared Imaging System (Li-COR Biosciences, Bad Homburg, Germany). Proteins were scanned using either the $700 \mathrm{~nm}$ or/and the $800 \mathrm{~nm}$ channels, depending on the secondary antibody used. 


\subsubsection{Antibodies used for Immunoblotting}

For identification of proteins by western blotting, the following antibodies were used according to the indicated dilutions in table:

Table 9: List of antibodies used for immunoblotting.

\begin{tabular}{|c|c|c|}
\hline Antibody & Dilution & Manufacturer \\
\hline \multicolumn{3}{|l|}{ Primary antibodies } \\
\hline$\alpha$-PSD-95 (mouse) & $1: 5000$ & Neuromab \\
\hline$\alpha$-PSD-93 (mouse) & $1: 5000$ & Neuromab \\
\hline$\alpha$-mortalin (mouse) & $1: 10000$ & Neuromab \\
\hline$\alpha-\beta$-tubulin (rabbit) & $1: 7500$ & Licor \\
\hline$\alpha$-GluN2A (rabbit) & $1: 5000$ & Epitomics \\
\hline$\alpha$-GluN2B (mouse) & 1:5000 & Neuromab \\
\hline$\alpha-G l u A 1$ (rabbit) & $1: 5000$ & Cell Signaling \\
\hline$\alpha$-GluA2 (mouse) & $1: 5000$ & Neuromab \\
\hline a-phospho-GluN2B (Y1472) (rabbit) & $1: 2500$ & Cell Signaling \\
\hline $\begin{array}{l}\alpha \text {-phospho-P38 MAPK (Thr180/ Tyr182) } \\
\text { (D3F9) (rabbit) }\end{array}$ & $1: 2000$ & Cell Signaling \\
\hline$\alpha$-Cleaved casp-3 (Asp175) (5A1E) (rabbit) & $1: 2000$ & Cell Signaling \\
\hline$\alpha$-Non-phospho-STEP (Ser221) (D74H3) & $1: 2000$ & Cell Signaling \\
\hline \multicolumn{3}{|l|}{ Secondary antibodies } \\
\hline a-mouse Alexa Flour 680 (goat) & $1: 15000$ & Odyssey Licor \\
\hline$\alpha$-rabbit Alexa Flour 800 (goat) & $1: 15000$ & Odyssey Licor \\
\hline
\end{tabular}




\subsubsection{Chemical Cross-linking of Cell surface proteins}

For quantification of the levels of proteins expressed on cell surface, a chemical cross-linking approach was taken by using a membrane-impermeable, bifunctional cross-linker bis(sulfosuccinimidyl)suberate (BS3) (Boudreau et al., 2012). For this mouse acute visual cortex slices were prepared from P20-P30 C57/BL/6 wild-type or PSD93 knockout mice in ice-cold sucrose cutting buffer $(87 \mathrm{mM} \mathrm{NaCl}, 2.5 \mathrm{mM} \mathrm{KCl}$, $0.5 \mathrm{mM} \mathrm{CaCl}_{2}, 1.25 \mathrm{mM} \mathrm{NaH}_{2} \mathrm{PO}_{4}, 25 \mathrm{mM} \mathrm{NaHCO}_{3}, 25 \mathrm{mM}$ - Glucose, $75 \mathrm{mM}$ Sucrose).

The animals were anesthetized using isoflurane and decapitated to take the brain out in ice-cold sucrose cutting buffer. $400 \mu \mathrm{m}$ thick whole brain slices were prepared from both hemisphere is sucrose cutting buffer using the Leica VT 1200S slicing device (Leica, Solmes, Germany). The slices were taken on a whatman no. 1 filter paper (Whatman, cat. no. 1001-070)soaked in ice cold sucrose cutting buffer. A chilled metal syringe barrel was used to take tissue punches from the layer $2 / 3$ of visual cortex slices and taken into $1 \mathrm{ml}$ of HEPES buffer solution $(1.2 \mathrm{mM} \mathrm{CaCl}, 20 \mathrm{mM}$ HEPES, $147 \mathrm{mM} \mathrm{NaCl}, 100 \mathrm{mM}$ dextrose, $\mathrm{pH}$ 7.4) spiked with $40 \mu \mathrm{l}$ of $52 \mathrm{mM} \mathrm{BS} 3$ stock solution, added just prior to adding the tissue. The tubes were incubated at $4{ }^{\circ} \mathrm{C}$ for $30 \mathrm{~min}$ on a rotator. The cross-linking reaction was quenched by adding $100 \mu 1$ of $1 \mathrm{M}$ glycine stock (1:10 dilution) and was incubated for another $10 \mathrm{~min}$ at $4{ }^{\circ} \mathrm{C}$ on the rotator. The tube was centrifuged at $14,000 \mathrm{~g}$ at $4^{\circ} \mathrm{C}$ for $2 \mathrm{~min}$ and the supernatant was quickly discarded and replaced with $200 \mu \mathrm{l}$ of cold lysis buffer $(10 \mathrm{mM}$ Tris-HCl, $150 \mathrm{mM} \mathrm{NaCl}, 10 \mathrm{mM}$ EDTA, 0.1\% SDS, 0.5\% Deoxycholate, 1\% NP-40, 150mM $\mathrm{NaF}, 1 \mathrm{mM} \mathrm{Na} \mathrm{VO}_{4}$, Complete Protease inhibitor (Roche)). Using a glass-glass tissue homogenizer, the punches were homogenized using 5-6 up and down strokes. The homogenized samples were further sonicated for $5 \mathrm{sec}$ at a setting of $4 \mathrm{~W}$, on ice, to avoid heating up of the samples. 2X SDS sample buffer was added to it and heated at $55^{\circ} \mathrm{C}$ for $15 \mathrm{~min}$ and stored at $-20^{\circ} \mathrm{C}$ until further use. 


\subsubsection{NMDA Neurotoxicity protocol}

To check the effect of NMDA induced neurotoxicity, I performed a NMDA challenge protocol on cortical cultures prepared from P0-P1 mice (Zhang et al., 2010). Cultures in 24-well culture plates were treated with $30 \mu \mathrm{M}$ NMDA in presence or absence of $10 \mu \mathrm{M}$ APV (added 10 min prior to NMDA treatment) for 1 hour at room temperature in a humid, $5 \%$ equilibrated $\mathrm{CO}_{2}$ reaction chamber. To block any APMA mediated excite-toxicity, $10 \mu \mathrm{M}$ NBQX was also added $10 \mathrm{~min}$ before the NMDA treatment was done. After 1 hour, first half of the media was changed with fresh feeding media, without any drugs, and this process was repeated once more to dilute out any residual drugs from the media. The plates were transferred back to $37^{\circ} \mathrm{C}$ incubator with $5 \%$ equilibrated $\mathrm{CO}_{2}$ concentration, for further 24 hours. Cells were washed with ice-cold PBS with $\mathrm{Mg}^{2+} / \mathrm{Ca}^{2+}$. Cells were harvested in 2X SDS sample buffer and heated upto $95^{\circ} \mathrm{C}$ for $10 \mathrm{~min}$. The tubes were centrifuged at high speed for 1 minute and stored at $-20^{\circ} \mathrm{C}$ for further use.

\subsubsection{Propidium lodide staining}

For staining cells that are undergoing apoptosis I performed Propidium iodide staining of dissociated neurons (Kruman et al., 1997). Dissociated neuronal cultures on precoated cover-slips were washed gently with ice-cold PBS and fixed at room temperature for 10 min using fixation solution (4\% PFA, 4\%sucrose). After fixation, the cells were washed three times with cold PBS for 5 min each at room temperature. $1 \mu \mathrm{lof}$ Propidium iodide ( $1 \mu \mathrm{g} / \mu \mathrm{l}$ stock) was added to each well of a 24 -well culture dish and incubated at room temperature for $10 \mathrm{~min}$ in dark. Cells were next washed for three times with cold PBS for 10 min each and mounted on glass slides with $80 \%$ glycerol. Images were acquired using a confocal laser-scanning microscope (514 nm excitation, Zeiss LSM 710) with a 40x oil-immersion objective. Images were acquired and processed using the software. 


\subsubsection{Drugs Used in cell culture and acute visual cortex punches}

All the drugs used are stored as powder at $4{ }^{\circ} \mathrm{C}$ or as re-suspended aliquots at $4 \%-20^{\circ} \mathrm{C}$ according to supplier's instruction.

Table 10: Drugs used in cell culture and acute visual cortex punches

\begin{tabular}{|c|c|c|c|}
\hline Drug Name & Concentration & Effect & Supplier \\
\hline NMDA & $30 \mu \mathrm{M}$ & $\begin{array}{l}\text { NMDA receptor } \\
\text { agonist }\end{array}$ & $\begin{array}{l}\text { Ascent Scientific/Abcam, } \\
\text { Germany }\end{array}$ \\
\hline APV & $10 \mu \mathrm{M}$ & $\begin{array}{l}\text { NMDA receptor } \\
\text { blocker }\end{array}$ & $\begin{array}{l}\text { Ascent Scientific/Abcam, } \\
\text { Germany }\end{array}$ \\
\hline PP2 & $10 \mu \mathrm{M}$ & $\begin{array}{l}\text { Src family Kinase } \\
\text { blocker }\end{array}$ & $\begin{array}{l}\text { Sigma-Aldrich (München, } \\
\text { Germany) }\end{array}$ \\
\hline NBQX & $5 \mu \mathrm{M}$ & $\begin{array}{l}\text { AMPA Receptor } \\
\text { blocker }\end{array}$ & $\begin{array}{l}\text { Ascent Scientific/Abcam, } \\
\text { Germany }\end{array}$ \\
\hline Ifenprodil & $10 \mu \mathrm{M}$ & $\begin{array}{l}\text { GluN2B Subunit } \\
\text { blocker }\end{array}$ & $\begin{array}{l}\text { Ascent Scientific/Abcam, } \\
\text { Germany }\end{array}$ \\
\hline $\mathrm{ZnSO}_{4}$ & $30 \mu \mathrm{M}$ & $\begin{array}{l}\text { GluN2A Subunit } \\
\text { blocker }\end{array}$ & Roth, Germany \\
\hline BS3 & $52 \mathrm{mM}$ & $\begin{array}{l}\text { Protein Cross- } \\
\text { linker }\end{array}$ & Applichem, Germany \\
\hline
\end{tabular}




\section{Results}

The role of PSD-93 in maintaining the levels of glutamatergic receptors still remains controversial. Some publications have shown that knockdown of PSD-93 has no effect on basal synaptic transmission in hippocampus as there are other PSD-MAGUKs, which could compensate for the role of PSD-93 (Carlisle et al., 2008; Elias et al., 2006a; McGee et al., 2001b). Other publications have shown that in hippocampus, even though genetic knockout of PSD-93 has no effect on synaptic currents, a RNAi mediated knockdown of PSD-93 can cause the same effect as acute absence of PSD95 for basal synaptic transmission, i.e., a 50\% reduction of AMPA currents (Elias et al., 2006a). Previous studies from our lab had also shown that in hippocampus, specific PSD-93 isoform have differential role in maintaining synaptic AMPA receptor strength (Krüger et al., 2013a). Further more, there had been mixed opinion as to the necessity of PSD-93 in maintaining synaptic level of NMDA currents. No effect of PSD-93 had been found on hippocampal NMDA current (Tao and Johns, 2006) whereas, in cortex, PSD-93 was reported to influence synaptic NMDA currents (Tao and Johns, 2006). 


\subsection{Only the surface levels but not the total level of NMDA receptors are reduced in PSD-93 knockout visual cortex synapses}

While some reports claim that PSD-93KO causes no change in the basal synaptic current (Elias et al., 2006a; McGee et al., 2001a) others say that PSD-93KO has reduced NMDA current (Tao and Johns, 2006). One more observation was that PSD-93 seem to have different functions in different brain region, for example, while in hippocampal synapse PSD-93KO has no effect on basal synaptic transmission (McGee et al., 2001a), in cortical synapse PSD-93 does seem to influence the synaptic NMDA currents (Tao and Johns, 2006). Thus to look for the brain area specific role of PSD-93, I took an alternate biochemical approach to quantify the surface level of AMPA and NMDA receptors in PSD-93KO mice visual cortex synapses. I used tissue punches from the L2/3 of visual cortex from wild-type or PSD-93KO mice in the age range of P20-P30. I performed a surface protein cross-linking protocol using BS3 to cross-link the cell surface proteins in these punches. Non-cross-linked samples were used to measure the total amount of a particular protein (protein expressed on the cell surface as well as internal to the cell). In the cross-linked sample lanes, we could observe a high molecular weight cross-linked protein aggregate, which was difficult to quantify (Figure 4A). So I we quantified the monomeric bands for each of the proteins. In the non-cross-linked lane, the monomeric band represented the total level of the protein, whereas in the cross-linked lane, it represented the internal level of the protein, which could not be cross-linked (Figure 4A). We quantified the level of surface expressed protein using the following formula:

$$
\text { Normalized surface level of protein }=1-\frac{\text { internal level of protein }}{\text { total level of protein }}
$$

For checking the membrane impenetrability of the cross-linker, BS3, we used both PSD-95 (Figure 4B) as well as $\beta$-tubulin proteins (Figure 4C (bottom)), both of which 
are intracellular proteins.

Three independent biological replicates were performed during this cross-linking experiment and were taken for the western blot analysis together to minimize the errors occurring in all the down-stream steps. $20 \mu \mathrm{g}$ of proteins were used for each western blot analysis and probed with $\alpha$-GluN2A, $\alpha$-GluN2B, $\alpha$-GluA1, $\alpha$-GluA2 and $\alpha$ - $\beta$-tubulin antibodies at their previously mentioned concentrations. For secondary antibody treatment, alexa $\alpha$-mouse 680 and $\alpha$-rabbit 800 antibodies were used for visualizing proteins in 700 and 800 channels of the odyssey Li-Cor scanner, respectively. 

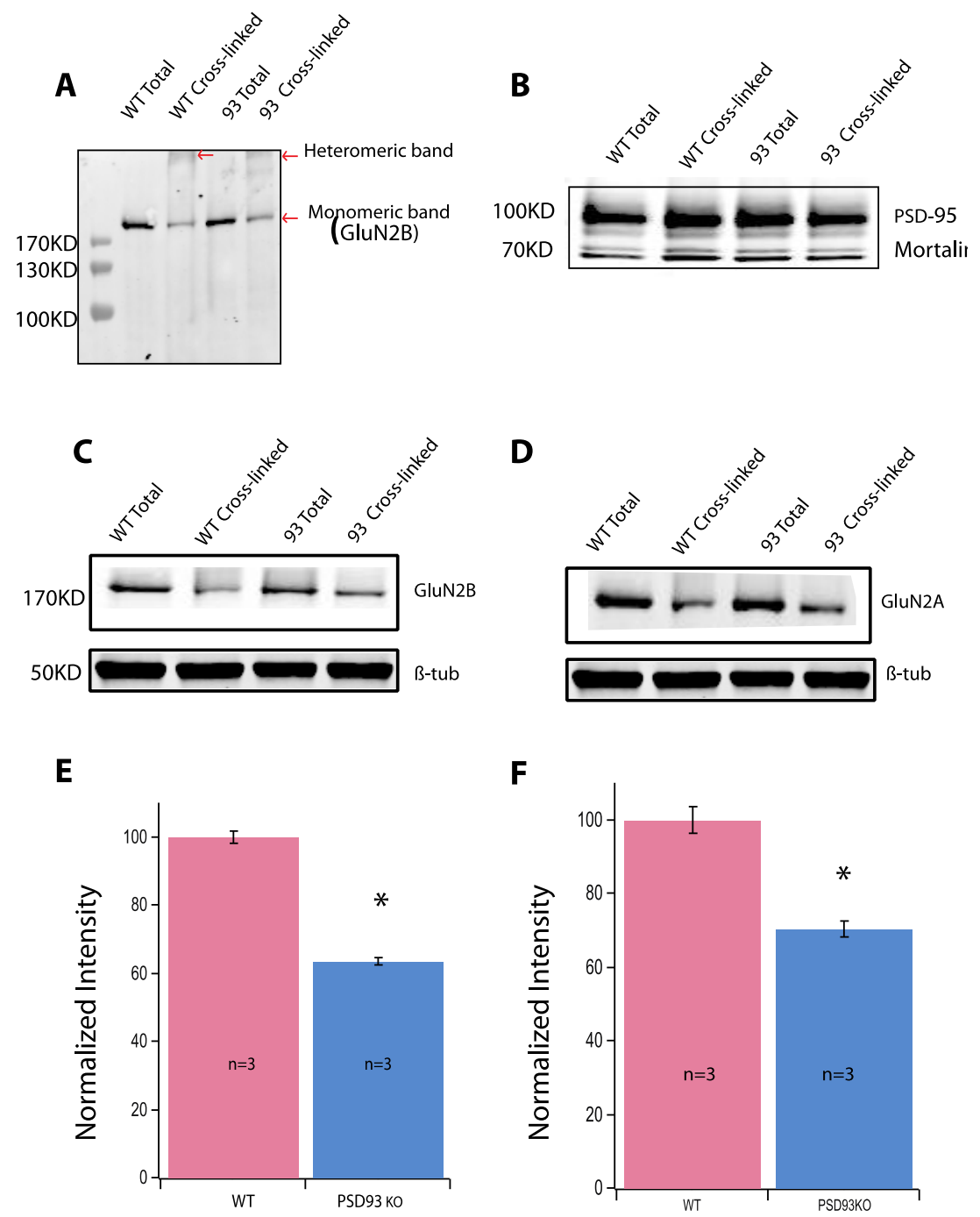

Surface GluN2B levels

Surface GluN2A levels

Figure 4: PSD-93KO has reduced surface NMDA receptor levels. A- Representative western blot from a surface cross-linking experiment. First and third lane are total level of protein in a non-crosslinked WT and PSD-93KO samples respectively; second and fourth lane are internal levels of protein in a cross-linked sample. Here, I used GluN2B, as an example. There is a high molecular weight fuzzy heteromeric band of cross-linked GluN2B (shown with red arrows), that was hard to quantify and a monomeric band of intra-cellular GluN2b (shown with blue arrow), which was quantified. B-PSD-95 antibody (top) was used as an intracellular control to show the membrane impenetrability of the crosslinking agent. C- GluN2B, D- GluN2A. $\beta$-tubulin shows no change in levels across the samples (bottom). E,F- Quantification for the surface level of GluN2B and GluN2A, respectively. The intensity of the surface levels of either GluN2B or GluN2A was normalized to the average WT surface level band intensity for the respective proteins. E- The PSD-93KO (shown in blue) showed a reduction from the WT level (shown in pink), $<<0.05$, T-Test. F- Quantification of the surface level of GluN2A. The PSD93KO (shown in blue) showed a reduction from the WT level (showed in pink), p<0.05, T-test. 
Both PSD-95 (Figure 4B) and $\beta$-tubulin (Figure 4C(bottom)) are intracellular proteins that are inaccessible for the cross-linker. Consequently, they showed no change in intensity between the total and the cross-linked lane. This reassures that BS3 can just cross-link proteins, which are expressed on the cell surface.

The first and the third lane represent the total amount of GluN2B or GluN2A, whereas the second and fourth lane represent the internal, non-cross-linked level of GluN2B or GluN2A from the wild-type and the PSD-93KO samples, respectively (Figure 4C and Figure 4D (top)).

I normalized the surface level of GluN2B in each sample to the average of the surface level of GluN2B in the WT samples. The GluN2B subunit of NMDA receptors showed a reduction in surface expression level (Wt. average $=100 \pm 1.878$, vs. PSD$93 \mathrm{KO}$ average $=63.44 \pm 1.100, \mathrm{p}<0.05, \mathrm{n}=3$. $($ Figure 4E)

Similarly, the GluN2A subunit of NMDA receptors showed a reduction in the surface expression level (Wt. average $=100 \pm 3.36$, vs. PSD-93KO average $=70.44 \pm 2.06$, $\mathrm{p}<0.05, \mathrm{n}=3$ ) (Figure 4F). B-tubulin didn't show any cross-linking and was equally distributed across all samples.

Thus the surface levels of both GluN2A and GluN2B subunits were reduced in the PSD-93KO animals.

Since the surface levels of NMDA receptor were reduced in PSD-93KO animals, we also wanted to check, whether the total level of NMDA receptors was reduced in PSD-93KO animals. For this we analyzed the level of GluN2A and GluN2B receptors from the non-cross-lanes (Figure 5A and B). 
A

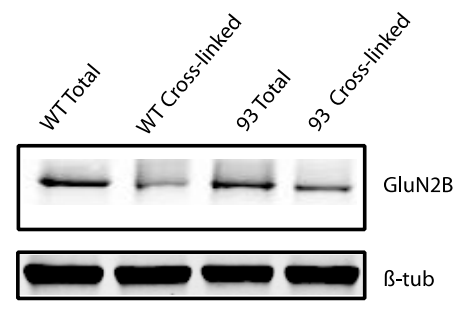

B

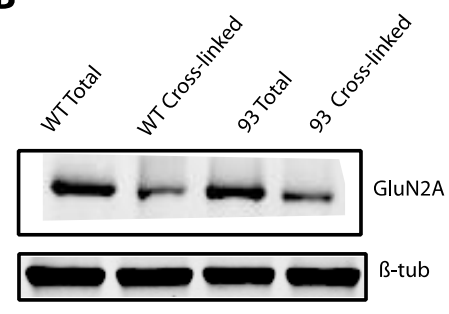

C

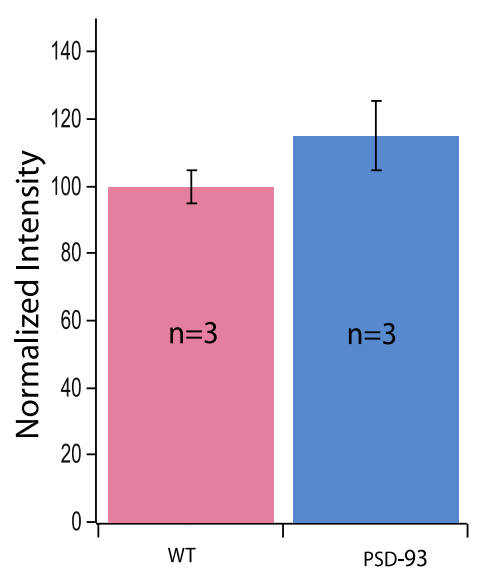

Total GluN2A levels wt vs. 93
D

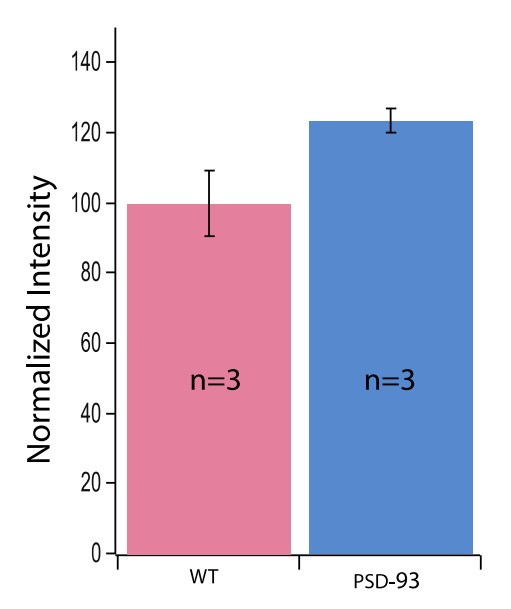

Total GluN2B levels wt vs. 93

Figure 5: Total level of NMDA receptors remain unchanged in PSD-93KO animals. A,B- representative western blot from a surface cross-linking experiment. First and third lane are total level of protein in a non-cross-linked WT and PSD-93KO samples respectively. A- GluN2A, B- GluN2B. $\beta$ tubulin shows no change in levels across the samples(bottom). C- Quantification for the surface level of GluN2A. The PSD-93KO (shown in blue) showed no significant reduction from the WT(shown in pink),p $>0.05$, T-Test. D- Quantification for the surface level of GluN2B. The PSD-93KO (shown in blue) showed no significant reduction from the WT (showed in pink).

We compared the total levels of GluN2A and GluN2B between the wild-type and PSD-93KO. Levels of both GluN2A and GluN2B remained unchanged in PSD-93KO animals (Average intensity of GluN2A: WT: $100 \pm 5.17$, PSD-93: $115.09 \pm 10.29$, $(\mathrm{p}=0.282$, n.s. $), \mathrm{n}=3$; GluN2B : WT: $100 \pm 9.55$, PSD-93 : $123.55 \pm 3.54,(\mathrm{p}=$ 0.119, n.s.), $\mathrm{n}=3$ )(Figure 5C and D). 


\subsection{Surface levels of AMPA receptors remain unchanged in PSD-93 knockout visual cortex synapses}

A

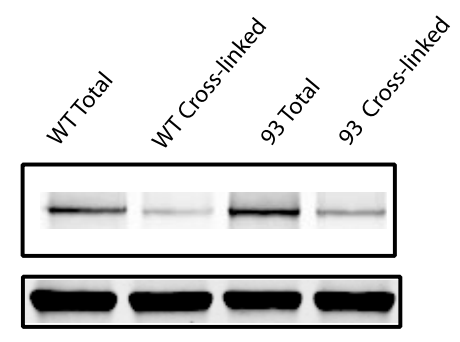

C

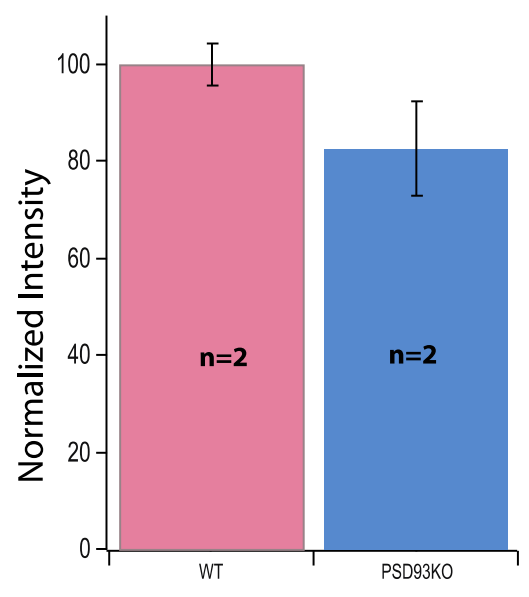

Surface GluA 1 levels
B

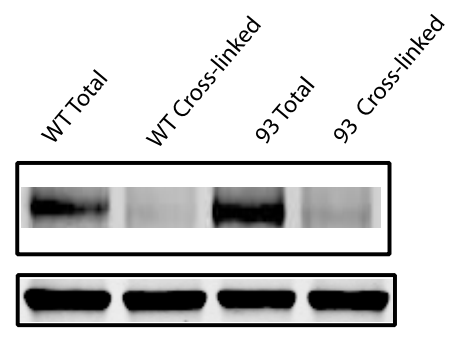

D

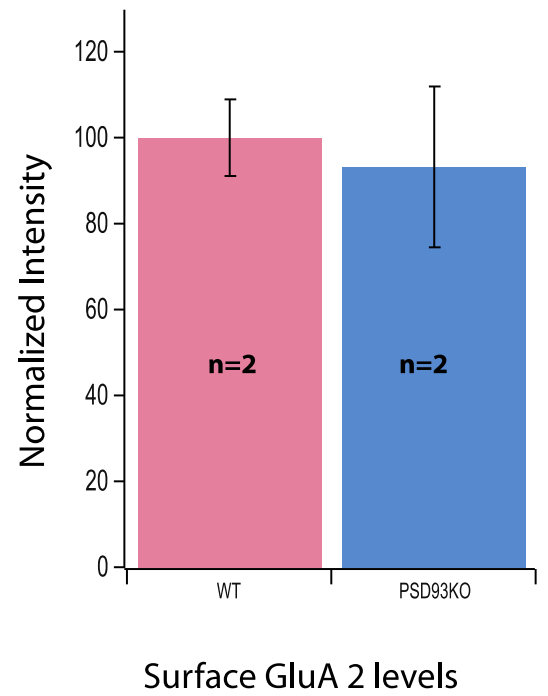

Figure 6: PSD-93KO showed no significant reduction in surface AMPA receptor levels. A,Brepresentative western blot from a surface cross-linking experiment. First and third lane are total level of protein in a non-cross-linked WT and PSD-93KO samples respectively; second and fourth lane are internal level of protein in a cross-linked sample. A- GluA1, B- GluA2. $\beta$-tubulin shows no change in levels across the samples (bottom). C- Quantification for the surface level of GluA1 and GluA2, respectively. The intensity of the surface levels of either GluA1 or GluA2 was normalized to the average WT surface level band intensity for the respective proteins. C- Quantification for the surface level of GluA1. The PSD-93KO (shown in blue) showed no significant reduction from the WT (shown in 
pink), $(\mathrm{p}=0.302$, n.s), T-Test. D- Quantification for the surface level of GluA2. The PSD-93KO (shown in blue) showed no reduction from the WT (showed in pink), ( $\mathrm{p}=0.791$, n.s, T-Test).

The first and the third lane represent the total amount of, whereas the second and fourth lane represent the internal, non-cross-linked amount of GluA1 or GluA2 from the WT and the PSD-93KO samples, respectively (Figure 6A and Figure 6B (top)). $\beta$ tubulin was used as an internal housekeeping protein (Figure 6 (bottom)).

When AMPA receptor levels were analyzed, the GluA1 subunit of AMPA receptors showed no reduction in surface expression level $(\mathrm{Wt}$. average $=100 \pm 4.49$, vs. PSD93KO average $=82.69 \pm 9.90, \mathrm{p}=0.302$ (n.s), $\mathrm{n}=2$, T-test) (Figure 6C)

The GluA2 subunit of AMPA receptors also showed no significant change in surface expression level $($ Wt. average $=100 \pm 9.16$, vs. PSD93KO average $=93.36 \pm 18.90$, $\mathrm{p}=0.791$ (n.s), n=2) ß-tubulin was used as an intracellular control for the cross-linking reactions and showed no cross-linking between different samples (Figure 6 A, B (bottom)).

\subsection{PSD-93 physically interacts with NMDA receptors in vivo}

Dlg-MAGUKs have been known to interact with glutamate receptors either directly (Kornau et al., 1995b; Niethammer et al., 1996) or via TARP proteins (Bats et al., 2007).Although PSD-93 had been reported to be essential in regulating AMPA receptor stabilization in mature synapses in conjunction with PSD-95 in hippocampus (Elias et al., 2006b) other brain regions like in spinal dorsal horn and forebrain showed that PSD-93 has no effect on AMPA receptor currents (Tao et al., 2003). Rather in spinal dorsal horn and forebrain, PSD-93 has been shown to be more important for NMDA receptor surface expression and synaptic function (Tao et al., 2003). Also, since in my experiments only the NMDA receptor surface expression pattern changed 
in the PSD-93 visual cortex, I wanted to check if in the visual cortex synapses PSD93 has direct physical interactions with NMDA receptors.

In order to do that, we performed a co-IP experiment. We used wild-type P20-P30 animals and dissected out punches from the layer $2 / 3$ of the visual cortex. $1 \mu \mathrm{g}$ of $\alpha$-PSD-93 antibody was as the primary antibody. A no antibody negative control was performed to rule out any non-specific interaction of the lysate proteins with the sepharose beads. Subsequent steps of co-immuno-precipitations were followed and finally using protein G-sepharose, the PSD-93 protein was pulled down. $10 \mu 1$ of the input was analysed along side of $25 \mu 1$ of the co-IP sample on the western blot. The membrane was probed with $\alpha$-PSD-93, $\alpha$-GluN2A and $\alpha$-GluN2B antibodies.

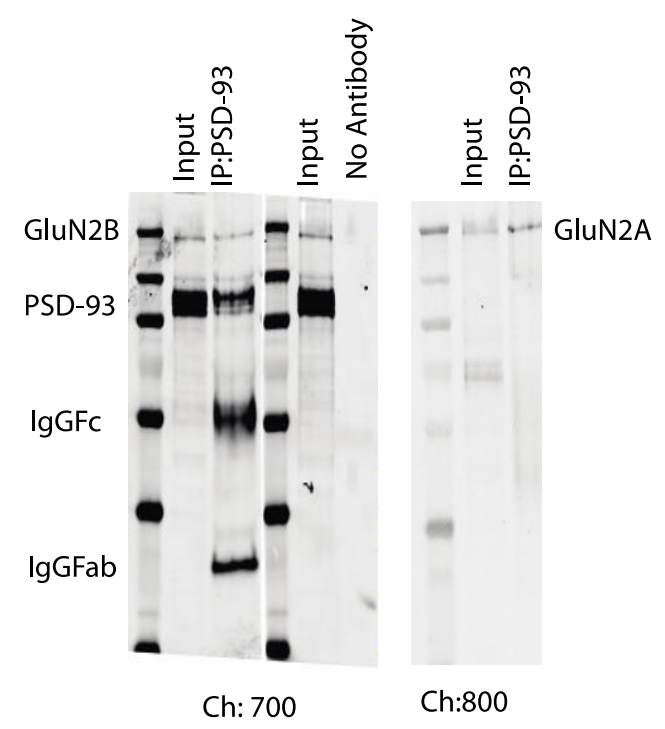

Figure 7: PSD-93 showed interaction with both GluN2A and GluN2B proteins in vivo. The representative western blot shows a strong band for PSD-93 in the lanes marked as 'Input'. The lane marked as IP:PSD-93, shows the Co-IP reactions where $\alpha$-PSD-93 antibody was used for immune-precipitation. Both GluN2B and GluN2A bands are marked in the picture. Both GluN2B and GluN2A bands are present in input as well as the IP:PSD-93 lanes, showing their interaction with PSD-93. In the co-IP lanes bands for the heavy chain (50KD) and light chain $(25 \mathrm{KD})$ are also visible. The 'No Antibody' lane shows no visible band for PSD-93 or any other proteins.

PSD-93 protein was identified at its expected molecular weight in both the input and the co-IP lanes (Figure 7). The GluN2B band was detected in the 700 channel in the 
input as well as the co-IP lane. The GluN2A band was detected in the 800 channel in the input as well as the co-IP lane. The no-antibody pull down didn't show bands for PSD-93 or any of the other proteins. Additional bands corresponding to the heavy chain (IgGFc) and the light chains (IgGFab) of the $\alpha$-PSD93 antibody, used for the immune-precipitation, was detected at $50 \mathrm{KD}$ and $25 \mathrm{KD}$ molecular weights respectively in the co-IP lane.

This experiment showed that PSD-93 has direct physical interaction with both GluN2A and GluN2B subunits of NMDA receptors in the synapses of layer 2/3 of mice visual cortex. This is supported by studies using X-ray crystallography to also find out physical reaction of PSD-93 with NMDA subunits (Fiorentini et al., 2013). Thus, PSD-93 might promote stabilization of GluN2A and GluN2B on the cell surface by physically interacting with them and in the PSD-93KO, due to lack of this interaction, there could be a reduced surface level of NMDA receptors.

\subsection{Surface level of phospho-GluN2B subunit is reduced in PSD-93 knockout synapses}

Previous reports have identified the Tyr1472 of GluN2B as one very important site to get phosphorylated (Chen and Roche, 2007). The phosphorylation of Tyr1472 prevents AP2 to bind to GluN2B molecules (Lavezzari et al., 2003; Roche et al., 2001). AP2 is an adaptor protein that aids in clathrin-mediated endocytosis of membrane proteins. Effectively, if the Tyr1472 stays phosphorylated, it will be inaccessible for endocytosis and hence there will be better retention of GluN2B on the membrane. Since, the PSD-93 mutants showed a lower level of surface GluN2B, I checked whether the phosphorylation level of GluN2B is altered in this mutant. 
A

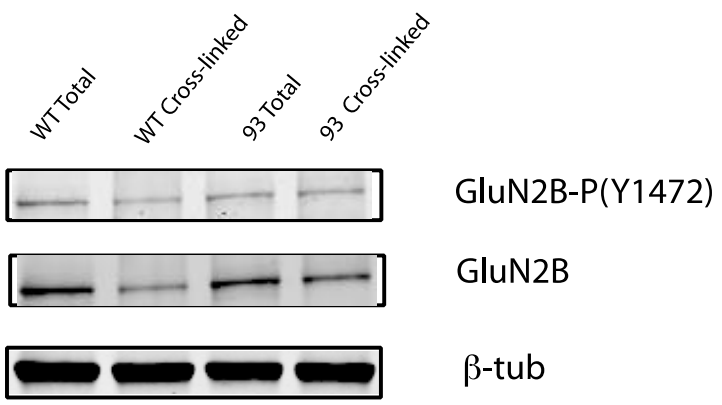

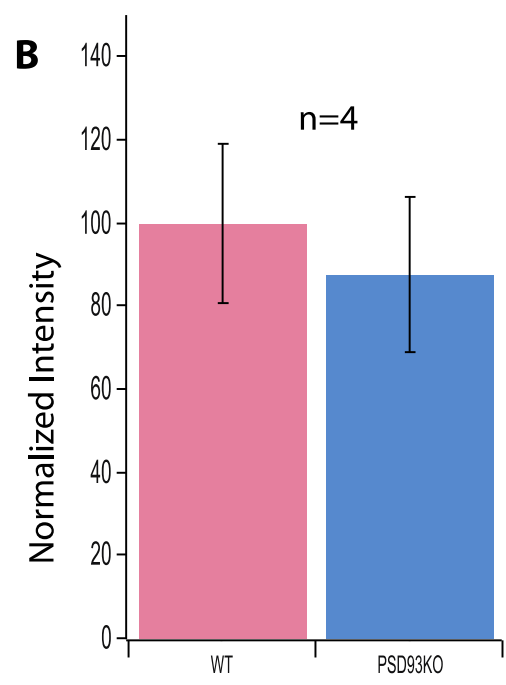

Total level of GluN2B-P

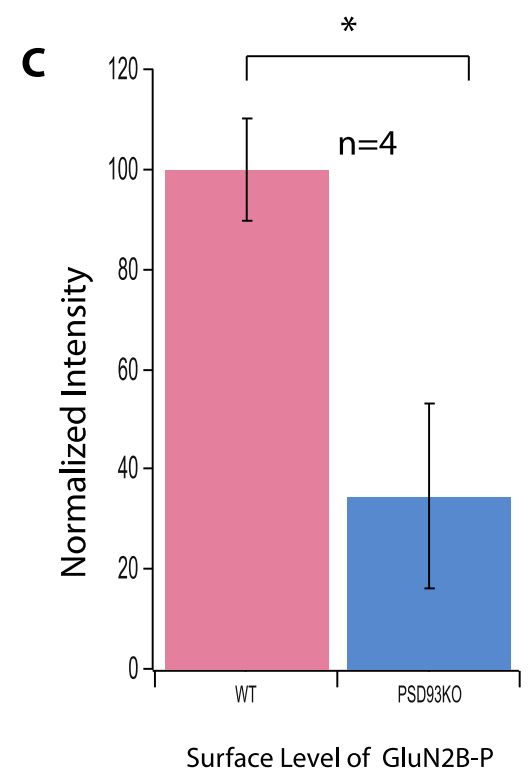

Figure 8: PSD-93KO has reduced level phosphorylated GluN2B on the cell surface. A-representative western blot from a surface cross-linking experiment. First and third lane are total level of protein in a non-cross-linked WT and PSD-93KO samples respectively; second and fourth lane are internal level of protein in a cross-linked sample. Top-Phospho-GluN2B (Y1472), middle- GluN2B, bottom- $\beta$ tubulin. $\beta$-tubulin shows no change in levels across the samples . B- Quantification for the ratio of total level of phospho-GluN2B. The PSD-93KO (shown in blue) showed no significant reduction from the WT(shown in pink), $\mathrm{p}=0.66$ (n.s) $\mathrm{n}=4$, T-Test. C- Quantification for the surface level of phosphoGluN2B. The PSD-93KO (shown in blue) showed significant reduction from the WT (showed in pink), $\mathrm{p}<0.05, \mathrm{n}=4, \mathrm{~T}-$ Test.

I used P20-P30 wild-type or PSD-93 mutant animals and performed a chemical crosslinking protocol with tissue punches from the layer $2 / 3$ of the visual cortex as before. Samples were used by western blot using the standard technique. The membrane was probed with $\alpha$-GluN2B and $\alpha$-Phospho-GluN2B (Y1472) antibodies. $\beta$-tubulin antibody was used as a intracellular control. 
The level of the monomeric GluN2B (Y1472) (top), GluN2B (middle) and $\beta$-tubulin (bottom) protein bands in the WT vs. the PSD-93KO was seen (Figure 8A).

I quantified the ratio of the total level of phospho-GluN2B (Y1472). This shows the population of the GluN2B that gets phosphorylated at its Tyr-1472 site. All band intensities were normalized to the average band intensity of the wild-type lane and expressed as a percentage. The graph shows, that the total level of phospho-GluN2B (Y1472) remains unchanged between WT and the PSD-93KO animals (WT average level $=100 \pm 19.30$, vs. PSD93KO average ratio $=87.64 \pm 18.76, \mathrm{p}=0.66, \mathrm{n}=4$ ) (Figure 8B).

I also quantified just the surface level of phospho-GluN2B (Y1472). This represented the percentage of phospho-GluN2B (Y1472), which was expressed on the cell surface alone. The graph showed reduction of this ratio in the PSD-93KO animals (WT average ratio $=100 \pm 10.27$, vs. PSD93KO average ratio $=34.80 \pm 18.44, \mathrm{p}>0.05, \mathrm{n}=4) . \beta$ tubulin level stays unaltered across all cross-linked or non-cross-linked samples (Figure 8C).

These results help us conclude that PSD-93 is not directly involved in the phosphorylation of GluN2B at Y1472 and that in its absence the total level of GluN2B phosphorylation remains unaltered. But since the surface percentage of GluN2B (Y1472) is reduced in the PSD-93 mutants, it shows that in absence of PSD-93 the distribution of the phosphorylated GluN2B changes. PSD-93 either stabilizes the phosphorylated form of GluN2B on cell surface or it helps the retention of GluN2B on the membrane by recruiting a kinase that does a local phosphorylation event at its Tyr1472 residue.

\subsection{The level of GluN2B receptor is reduced in PSD-93 cortical but not in hippocampal cultures}

NMDA receptors had long been associated with glutamate induced ischemic brain injuries (Simon et al., 1984). I also observed a reduction in the surface expression of NMDA receptors in the PSD-93KO visual cortex (Figure 4). To test if the NMDA 
Results $\mathrm{P}$ a g e $\mid \mathbf{5 1}$

induced neurotoxicity is also reduced in these animals, I needed to switch to dissociated neuronal culture, for its ease of performing a NMDA challenge experiment. But first I needed to confirm whether, in the PSD-93KO dissociated cortical cultures, the surface levels of NMDA receptors are altered, especially since I was using the whole cortex to make the dissociated culture instead of just the visual cortex. Since there are apparent discrepancies in the role of PSD-93 between hippocampus and cortex, as discussed earlier, I also checked the level of NMDA receptors in hippocampal neurons. For this experiment, I made cortical or hippocampal dissociated cultures from P0-P1 wild-type and PSD-93KO mice.

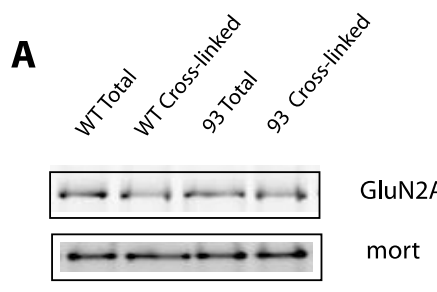

C

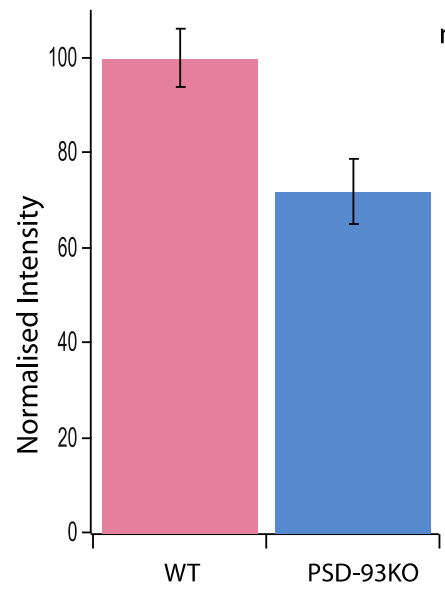

Surface GluN2A

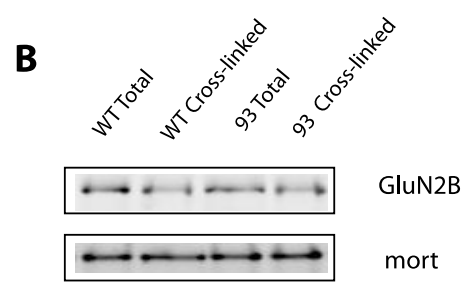

D

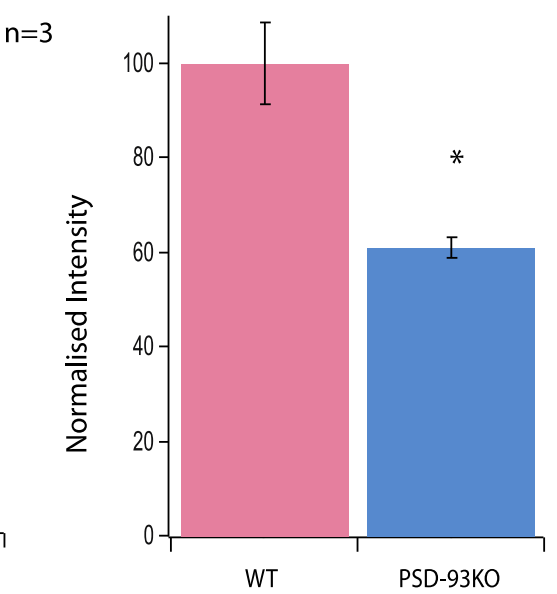

Surface GluN2B

Figure 9: PSD-93KO has reduced surface NMDA receptor levels in cortical culture. A,B- representative western blot from a surface cross-linking experiment. (Top) First and third lanes are total level of protein in a non-cross-linked WT and PSD-93KO samples respectively; second and fourth lanes corresponds to the internal level of protein in a cross-linked sample. A- GluN2A, B- GluN2B. Mortalin shows no change in levels across the samples (bottom). C- Quantification for the surface level of GluN2A. The PSD-93KO (shown in blue) showed a trend towards reduction from the WT level (shown in pink) but didn't reach significance, $\mathrm{p}=0.193$ (n.s), n=3, T-Test. D- Quantification of the 
surface level of GluN2B. The PSD-93KO (shown in blue) showed reduction from the WT levels (showed in pink), $\mathrm{p}<0.05, \mathrm{n}=3$, $\mathrm{T}$-test.

Surface protein cross-linking was performed on DIV 14 cortical cultures, using BS3 to a final concentration of $52 \mathrm{mM}$ and for the control wells DMFO was added (vector). Experiments were performed on three independent batches of culture for each reaction condition and analyzed by western blotting as before. Blots were probed with $\alpha$-GluN2A, $\alpha$-GluN2B and mortalin antibodies at their afore mentioned concentrations.

The monomeric GluN2A band could be seen in the wild-type and PSD-93KO noncross-linked and cross-linked lanes respectively. The band in the non-cross-linked lane represents the total amount of GluN2A in the cells, while the one in the crosslinked lane represents the internal level of GluN2A, which was not cross-linked (Figure 9A (top)). Mortalin, a member of the Hsp70 family of chaperones, was used as an intra-cellular protein control (bottom).

Quantification was done by normalizing all GluN2A surface level values with the average of the WT surface level value of GluN2A (Figure 9C). The graph showed a strong trend of reduction in the level of GluN2A in the PSD-93Ko culture but didn't reach significance (GluN2A average surface. level in WT: $100 \pm 6.05$, PSD-93KO: $71.70 \pm 15.11, n=3,(p=0.193$, n.s.), T-Test). Mortalin was equally distributed across samples.

We see the monomeric GluN2B band in the WT and PSD-93KO non-cross-linked and cross-linked lanes respectively. The band in the non-cross-linked lane represents the total amount of GluN2B in the cells, while the one in the cross-linked lane represents the internal level of GluN2B, which was not cross-linked (Figure 9B).

Quantification was done by normalizing all GluN2B surface level values to the average of the WT surface level value of GluN2B. The graph shows a significant reduction in the surface level of GluN2B in the PSD-93KO culture (GluN2B average surface. levels in WT: $100 \pm 8.68$, PSD-93KO: $60.90 \pm 6.97, \mathrm{n}=3, \mathrm{p}<0.05$, T-Test). 
Mortalin, used as the intra-cellular control, didn't show any significant change across samples (Figure 10D).

I performed a surface protein cross-linking on the DIV 14 hippocampal cultures as well and samples from three independent batches of cultures were analyzed by western blot.

A
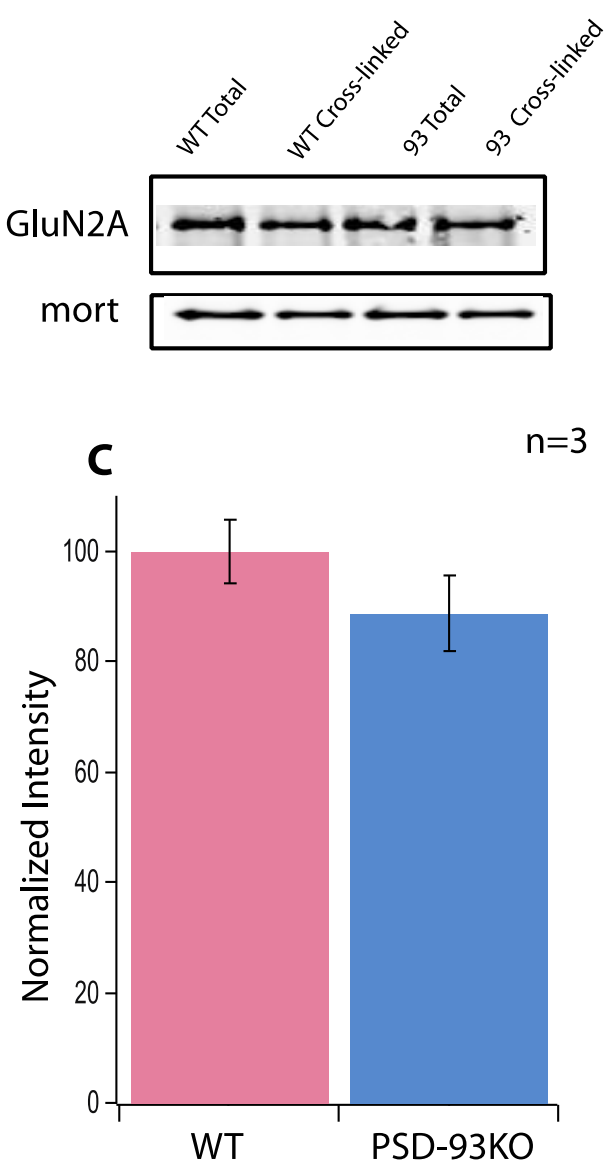

Surface GluN2A levels
B

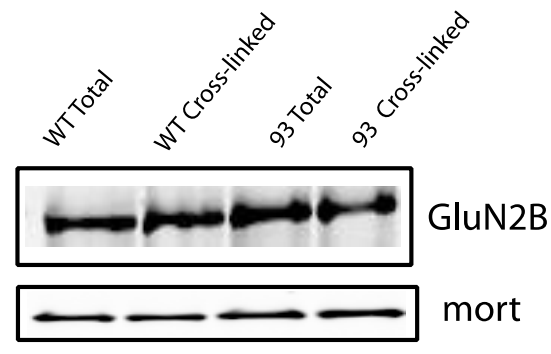

D

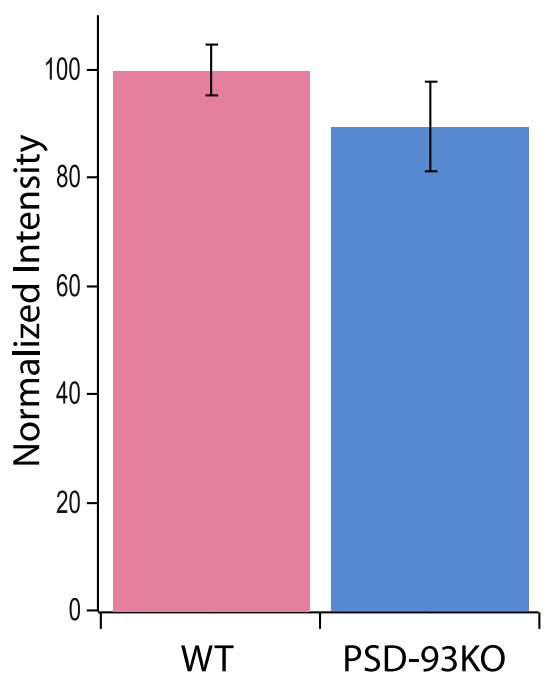

Surface GluN2B levels

Figure 10: PSD-93KO has no change in surface NMDA receptor levels in hippocampal culture. A,B- representative western blot from a surface cross-linking experiment. (Top) First and third lanes are total level of protein in a non-cross-linked WT and PSD-93KO samples respectively; second and fourth lanes corresponds to the internal level of protein in a cross-linked sample. A- GluN2A, BGluN2B. Mortalin shows no change in levels across the samples (bottom). C- Quantification for the surface level of GluN2A. The PSD-93KO (shown in blue) showed no change from the WT surface level (shown in pink), $p=0.27$ (n.s), $n=3$, T-Test. D- Quantification of the surface level of GluN2B. The 
PSD-93KO (shown in blue) showed no change from the WT surface levels (showed in pink), $p=0.34$, $\mathrm{n}=3$, T-test.

We see the monomeric GluN2A band in the wild-type and PSD-93KO non-crosslinked and cross-linked lanes respectively. The band in the non-cross-linked lane represents the total amount of GluN2A in the cells, while the one in the cross-linked lane represents the internal level of GluN2A, which was not cross-linked (Figure 10A (top)). Mortalin, a member of the Hsp70 family of chaperones, was used as an intracellular protein control (bottom).

Quantification was done by normalizing all GluN2A surface level values with the average of the WT surface level value of GluN2A (Figure 10C). The graph showed no reduction in the level of GluN2A in the PSD-93Ko culture in comparison to the WT (GluN2A average surface. level in WT: $100 \pm 5.80$, PSD-93KO: $88.61 \pm 6.71, \mathrm{n}=3$, $(p=0.270$, n.s.), T-Test). Mortalin was equally distributed across samples.

We see the monomeric GluN2B band in the WT and PSD-93KO non-cross-linked and cross-linked lanes respectively. The band in the non-cross-linked lane represents the total amount of GluN2B in the cells, while the one in the cross-linked lane represents the internal level of GluN2B, which was not cross-linked (Figure 10B).

Quantification was done by normalizing all GluN2B surface level values to the average of the WT surface level value of GluN2B. The graph shows no change in the surface level of GluN2B in the PSD-93KO culture (GluN2B average surface. levels in WT: $100 \pm 4.85$, PSD-93KO: $89.30 \pm 8.404, n=3, p=0.346$, T-Test). Mortalin, used as the loading control, didn't show any significant change across samples (Figure 10D). 


\subsection{Linking PSD-93, NMDA receptors and Apoptosis.}

\subsubsection{NMDA receptors are required for maintaining a balance between Neuronal Cell survival and neuronal Apoptosis.}

NMDA receptors signaling had been proposed to be one major mechanism for the choice a cell has to make between survival against apoptosis (Choi, 1988b; Tu et al., 2010b). Our results from previous experiments indicated a reduction of NMDA receptor subunits in PSD-93KO animals as well as primary cultures. Thus, we were curious to know, if absence of PSD-93 has any effect on the survival of neurons upon neurotoxic insults. To check this idea we first established a NMDA neurotoxic protocol. Cleaved casp-3 is a class of cysteine protease known to be an effector molecule produced during the later stages of apoptosis (Cohen, 1997; Porter and Jänicke, 1999). Hence, production of cleaved casp-3 was used as a marker for apoptosis.

\subsubsection{Increased cleaved casp-3 indicated increased cell mortality}

Though our model takes the level of cleaved casp-3 as a measure of apoptosis, active caspases were reported in non-apoptotic cells, as well (Campbell and Holt, 2003; Gilman and Mattson, 2002; Kuo et al., 2006; Williams et al., 2006; Yuan, 2006). There had been also reports claiming that increased caspase-3 levels may actually promote LTD in neurons by cleaving GluA1 receptors in synapses, rather than triggering a cell death pathway (Chan et al., 1999; Glazner et al., 2000; Schnell et al., 2002). Though the protocol adopted in those studies ( $\mathrm{Li}$ et al., 2010) for NMDA treatment to induce active caspase formation were distinctly different from ours (Zhang et al., 2010), we still wanted to be sure that the increased level of cleaved casp-3 actually measured cellular mortality and not just LTD in cortical cultures.

For this experiment we used a staining protocol using propidium iodide (PI). PI is a DNA binding dye that intercalates and measures nuclear condensation and fragmentation using fluorescence. PI in general is impermeable to the cell membrane. But during advanced stages of apoptosis, the cell membrane loses its integrity and becomes 
permeable to PI, which then goes and binds to the already fragmented nuclear DNA, giving it a characteristic punctate pattern.

A

NMDA $(30 \mu \mathrm{M}), \mathrm{NBQX}(10 \mu \mathrm{M})$

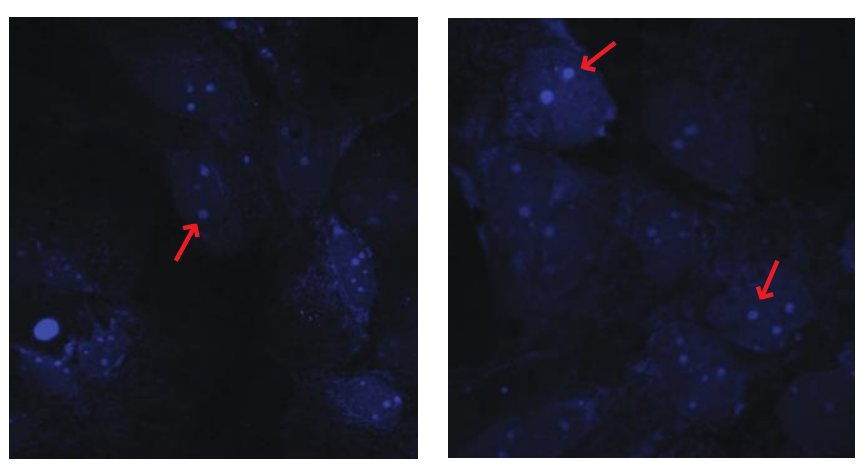

B NMDA $(30 \mu \mathrm{M}), \mathrm{NBQX}(10 \mu \mathrm{M}), \mathrm{APV}(10 \mu \mathrm{M})$
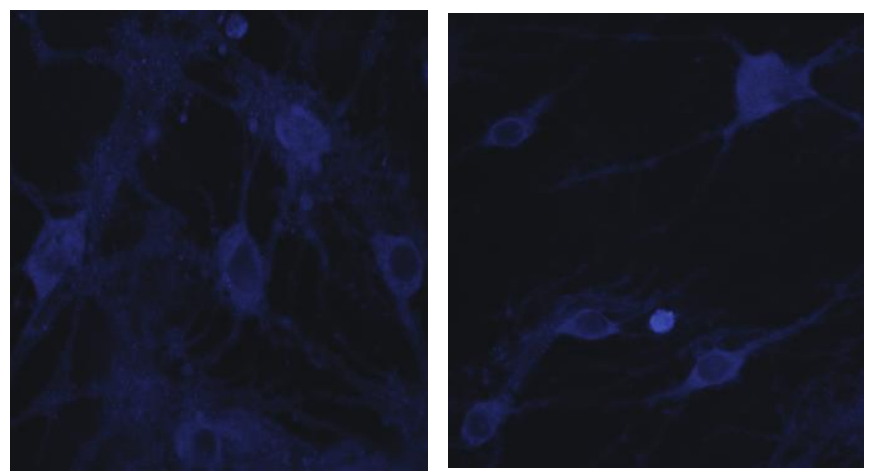

Figure 11: PI staining of NMDA challenged cortical cultures shows cell death. A, B- Two representative fields of view under 40X magnification of NMDA treated neuronal cultures. A- Cortical neurons treated with $30 \mu \mathrm{M}$ NMDA and $10 \mu \mathrm{M}$ NBQX (to block AMPA receptor responses). The pictures show apoptotic neurons with damaged cell membranes and bright punctate structures in the nucleus (marked with red arrows), which are fragmented pieces of chromosomal DNA. B- Cortical neurons treated with $30 \mu \mathrm{M}$ NMDA, $10 \mu \mathrm{M}$ APV and 10 $\mu \mathrm{M}$ NBQX. The APV treatment could completely block the membrane damage and punctate nuclear structures caused by the NMDA treatment.

We used DIV 17 wild-type cortical cultures and performed a NMDA challenge experiment with $30 \mu \mathrm{M}$ NMDA for 1 hour, either in presence or absence of $10 \mu \mathrm{M}$ APV. $10 \mu \mathrm{M}$ NBQX was always used to keep the AMPA receptors blocked from being activated. After 24 hours of incubation, we performed the PI staining protocol on the cultures. 
Results $\mathrm{P}$ a g e $\mid \mathbf{5 7}$

Figure 11A showed two representative fields of view under $40 \mathrm{X}$ magnification which revealed, that after 24 hours of NMDA challenge, the cortical neurons manifested clear signs of damaged cell membrane. The cells lost their typical shape and the nucleus also showed characteristic punctate structures that represent condensed and fragmented chromosomal DNA (marked with red arrows).

The two representative fields of view showed that by blocking NMDA receptor activity using APV, we could block the apoptotic effects of the NMDA challenge experiment. The cells looked healthy and there was little to no staining of the nuclear DNA (Figure 11B).

The above experiment proved that our NMDA challenge experiment and the corresponding elevated levels of cleaved casp-3, was directly co-related to increased neuronal mortality.

\subsubsection{Dose response Curve for NMDA induced excito-toxicity on cortical culture}

To find the optimal dose of NMDA that was necessary to evoke a neurotoxic effect on wild-type DIV 17 cortical neuronal cultures, we used different concentrations of NMDA ranging from $0-100 \mu \mathrm{M}$. Cells were treated with $10 \mu \mathrm{M}$ of NBQX to block all AMPA receptor responses. 


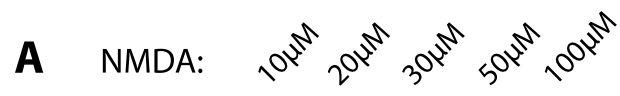
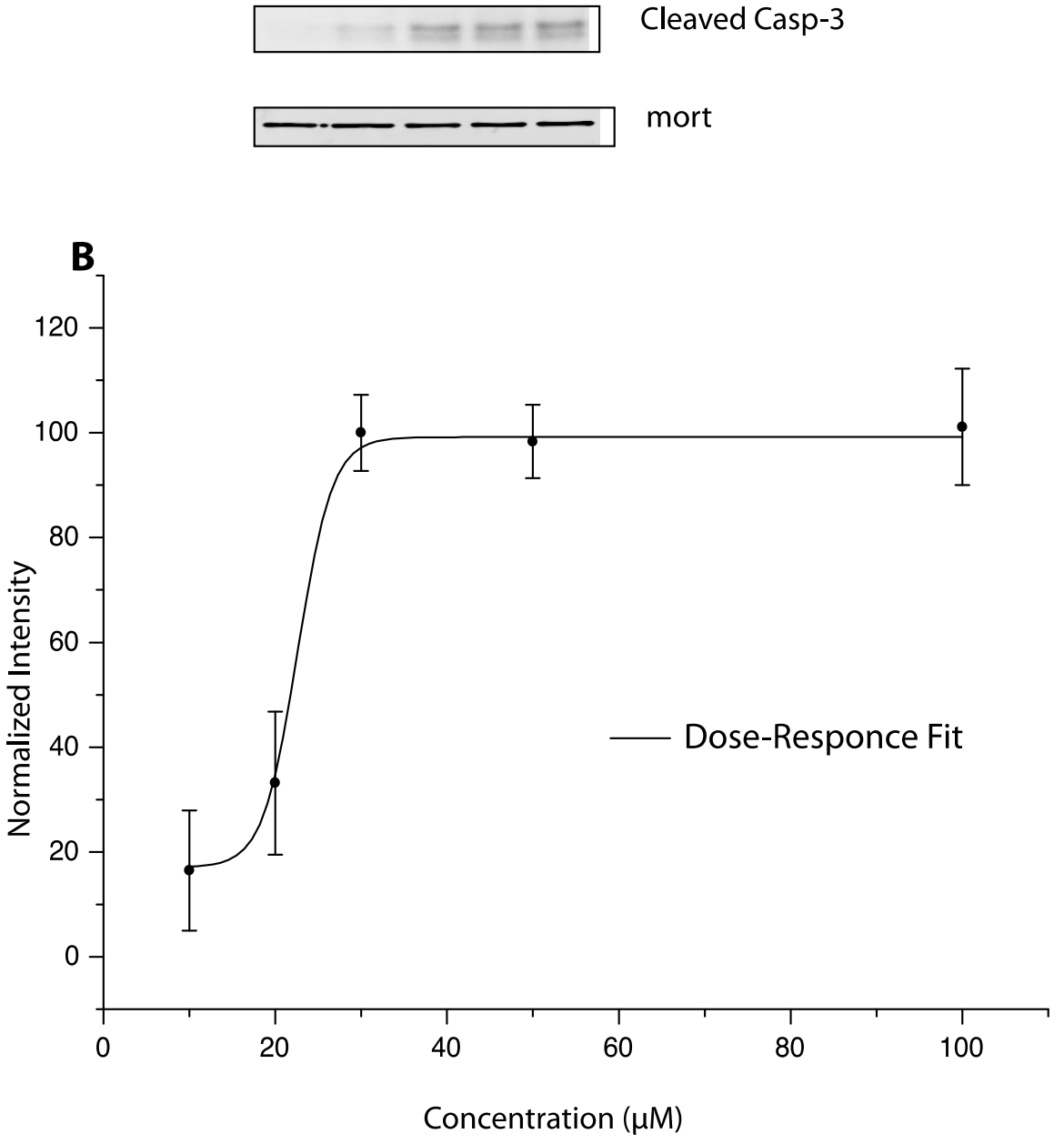

Figure 12: Dose-response curve of NMDA treatment on cortical neurons. A- Representative western blot image for the level of cleaved casp-3 upon NMDA treatment (Top). Concentration of NMDA are varied from $10 \mu \mathrm{M}$ to $100 \mu \mathrm{M}$. Mortalin levels remain unchanged over different samples lanes (bottom). B- Quantification of the concentration of NMDA (in $\mu \mathrm{M}$ ) vs. intensity of cleaved casp-3 produced(normalized to the $30 \mu \mathrm{M}$ NMDA dose). The level of cleaved casp-3 produced slowly increases until it reaches saturation after $30 \mu \mathrm{M}$ NMDA concentration.

Primary antibodies against cleaved casp-3 and mortalin were used in their aforementioned concentrations.

I saw a gradual increase in the level of cleaved caspase-3 with increasing NMDA concentration. All the cleaved casp-3 band values were normalized against the average intensity of the $30 \mu \mathrm{M}$ NMDA treatment lane (Figure 12A). 
I quantified the levels of cleaved casp-3 by normalizing it to the average band intensity in the $30 \mu \mathrm{M}$ NMDA treatment lane. The dose-response curve shows that the increase in the level of cleaved casp-3 reached a plateau after $30 \mu \mathrm{M}$ of NMDA concentration (Average normalized intensity for $10 \mu \mathrm{M}$ NMDA: $16.43 \pm 11.46,20 \mu \mathrm{M}$ : $33.14 \pm 13.64,30 \mu \mathrm{M}: 100 \pm 7.24,50 \mu \mathrm{M}: 98.32 \pm 7.03,100 \mu \mathrm{M}: 101.11 \pm 11.12$, $\mathrm{n}=3$ ). Mortalin was used as a loading control, which didn't vary across the samples (Figure 12B).

Thus, a concentration of $30 \mu \mathrm{M}$ NMDA is sufficient to induce maximal level of neuro-toxicity to cortical neurons. For all further experiments for NMDA induced neurotoxicity, $30 \mu \mathrm{M}$ was used as the standard NMDA dose, unless mentioned otherwise.

\subsubsection{Time-course for NMDA induced excito-toxicity on cortical culture}

To check the time course for an NMDA mediated neurotoxic challenge, we chose different time points of NMDA treatment to monitor it's effect on the mortality of cortical neurons. We took wild-type DIV 17 cortical neuronal cultures and subjected them to $30 \mu \mathrm{M}$ NMDA treatment. We chose time points of $0 \mathrm{~min}, 10 \mathrm{~min}, 20 \mathrm{~min}, 30$ min, 1 hour, 4 hours and 8 hours for NMDA treatment.

Biological replicates from independent batches of neuronal cultures were performed and samples were stored until further processed with the western blotting.

Primary antibodies against cleaved casp-3 and mortalin were used in their usual concentrations. 
A

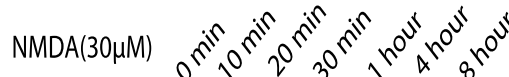

Cleaved Casp-3

- - - - mort

\section{B}

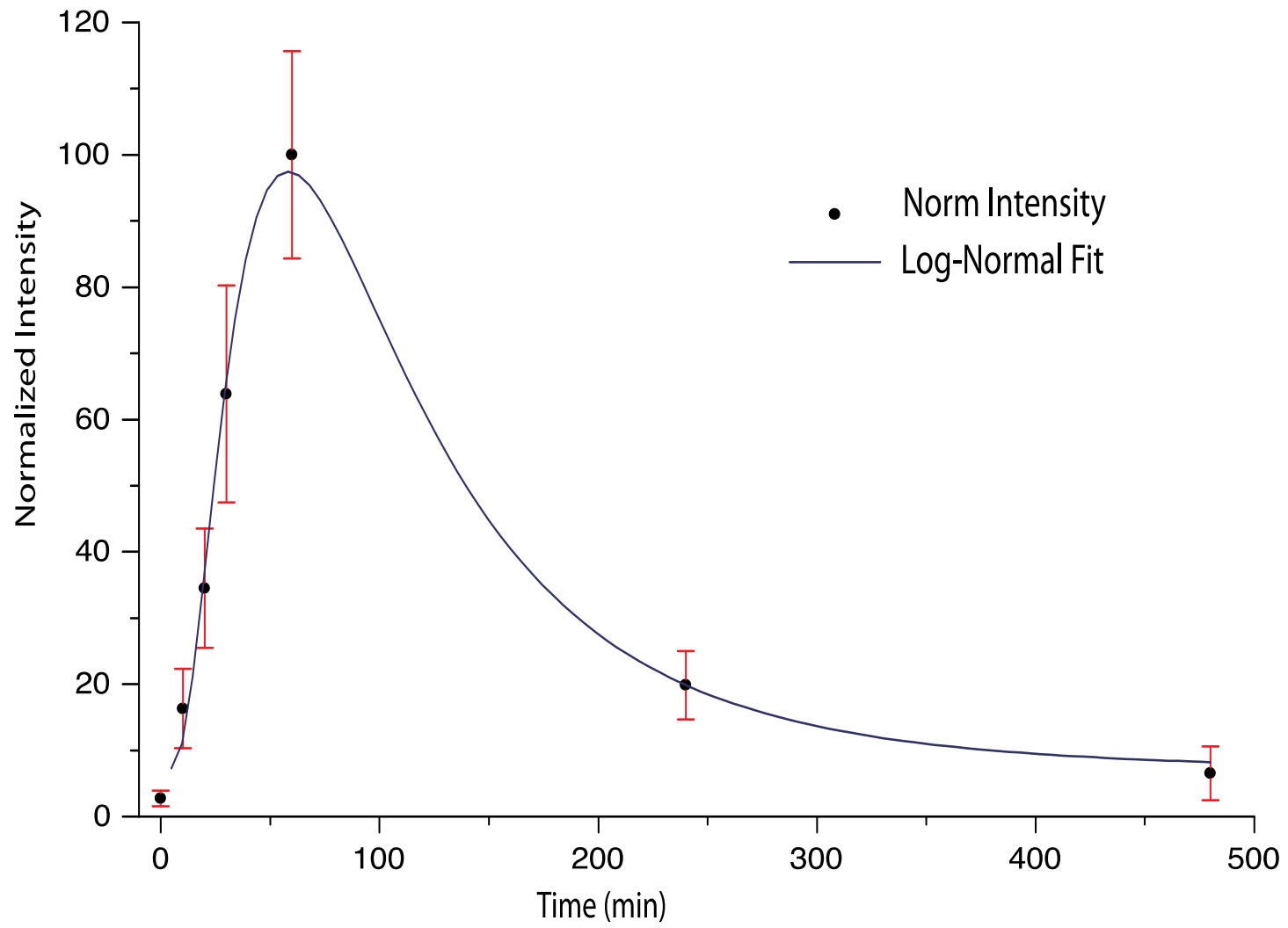

Figure 13: Time course for NMDA treatment on cortical neurons. A- The cleaved casp-3 band at increasing time of incubation of cortical neurons with $30 \mu \mathrm{M}$ of NMDA between 0 min till 8 hours (top). Mortalin concentration remains unchanged over all time points (bottom). B- Quantification of time vs. concentration of cleaved casp3 (normalized against the 1 hour time point concentration). The level of cleaved casp-3 steadily increases till the 1 hour time point but drops for the 4 hour and 8 hour time points. The distribution can be fit to a lognormal distribution curve.

The $\alpha$-cleaved caspase- 3 antibody, which is a marker for apoptosis, showed a gradual increase in its level till 1 hour of NMDA treatment. But upon NMDA treatment for extended time points of 4 hours or more, the level of cleaved casp-3 gradually decreased (Figure 13A (top)). The level of mortalin remained unchanged across the samples (Figure 13A (bottom)). 
Results P a g e | 61

For quantification, the cleaved casp-3 band values were normalized against the average intensity of the 1hour NMDA treatment lane. The intensities of cleaved casp-3, saw a gradual increase till 1 hour of $30 \mu \mathrm{M}$ NMDA treatment, after which it started to decline (Average normalized intensity for $30 \mu \mathrm{M}$ NMDA treatment for $0 \mathrm{~min}: 2.71 \pm$ 1.18, 10 min: $16.30 \pm 6.00,20 \mathrm{~min}: 34.51 \pm 9.00,30 \mathrm{~min}: 63.85 \pm 16.38,1$ hour: 100 \pm 15.65 , 4 hour: $19.83 \pm 5.16,8$ hour: $6.52 \pm 4.07, n=3$ ). Though the level of cleaved casp-3 in 4 hours and 8 hours time point did look much reduced, but the cells on microscopic observation showed signs of apoptotic damage. Upon plotting the time vs. intensity curve, we saw that it followed a lognormal distribution. Mortalin was used as a loading control, which didn't vary across the samples.

The results showed that upon activation of NMDA receptors for up till 1 hour the mortality of cortical neurons increased. Keeping NMDA receptors activated for an extended period of time ( 4 hours or more) decreased the level of cleaved casp-3. But even at those time points, the cells suffered apoptotic damage. This could indicate, that the first phase of NMDA receptor activation may trigger caspase-3 mediated programmed cell death. But continued activation of the NMDA receptors might lead to activation of caspase-independent forms of apoptosis (Borner and Monney, 1999; Stoka et al., 2001). Thus, as 1 hour of $30 \mu \mathrm{M}$ NMDA treatment was sufficient to cause the highest degree of cell death by the caspase dependent pathway, for all further experiments of NMDA challenge, I chose 1 hour incubation of as standard time point, unless mentioned otherwise.

\subsubsection{PSD-93 deletion causes reduced neuronal mortality in cortical cells upon NMDA challenge}

It had been proposed that NMDA receptors are crucial in maintaining a balance between neuronal survival and apoptosis. While GluN2B receptor containing NMDA receptors had been attributed to be pro-apoptotic in nature (Hardingham et al., 2002; Vanhoutte and Bading, 2003), the GluN2A receptors are supposed to be pro-cell survival (Anastasio et al., 2009; DeRidder et al., 2006). Since in the PSD-93KO animals the surface levels of GluN2B receptors were reduced, I wanted to examine if this had an effect on the NMDA mediated neurotoxicity levels in PSD-93KO cultures. 
Results $\mathrm{P}$ a g e | 62

For this experiment, I took wild-type and PSD-93 knockout cultures at DIV 17. We challenged the cells with $30 \mu \mathrm{M}$ of NMDA for 1 hour in presence or in absence of $10 \mu \mathrm{M}$ APV, which had been added 5 min prior to the NMDA treatment. Cells were harvested after 24 hours. The experiments were repeated with independent batches of cortical cultures and results were analyzed on western blotting for the levels of cleaved casp-3.

\section{A}

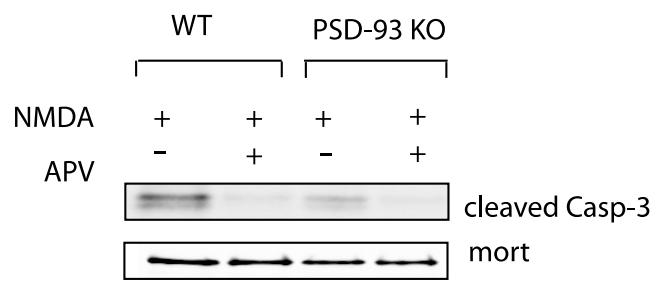

B

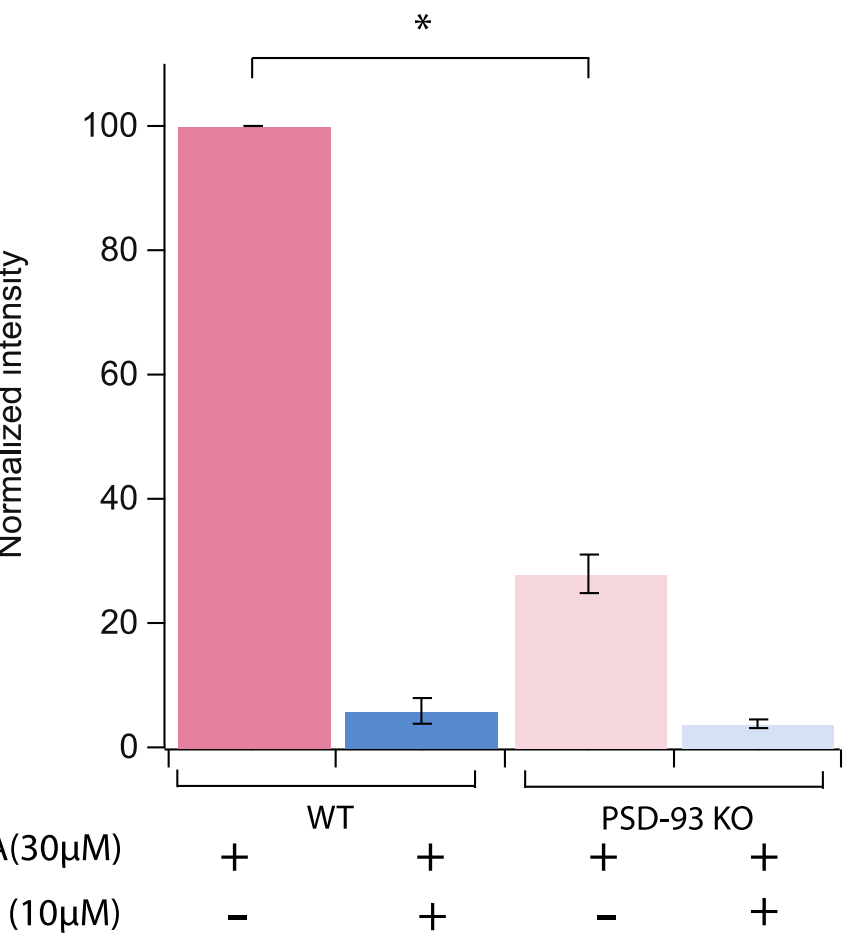

Figure 14: PSD-93KO cultures have reduced mortality upon NMDA challenge. A-Representative western blot showing the cleaved casp-3 level in WT vs. PSD-95KD culture in presence or in absence of APV. The cleaved casp-3 protein (top) could be blocked by APV treatment and the mortalin, stays equally distributed across all the samples (bottom). B- Quantification of the level of cleaved casp-3. The level of cleaved casp-3 in the wild-type (shown in deep pink) was reduced significantly in the PSD-93KO cultures (shown in light pink), $\mathrm{p}<0.05$, T-Test. APV could block the effect of NMDA in both wild-type (shown in blue) as well as in PSD-93KO cultures (shown in light blue). 
The band intensity for the cleaved casp-3 protein in the WT samples, treated with NMDA, was significantly reduced in the PSD-93KO treated with NMDA. The cleaved casp-3 levels could be almost entirely blocked in presence of APV in both the WT as well as PSD-93KO (Figure 14A (top)). The PSD-93KO culture also showed reduced signs of apoptotic damage when observed under microscope. Mortalin showed no significant difference across samples (Figure 14A (bottom)).

To account for the difference in level of protein across different blots I normalized each band intensity to its corresponding WT NMDA treated band intensity and then expressed it as a percentage. So the wild-type NMDA treated sample has no variance as it is always $100 \%$ and the other conditions are in relative to it. The PSD-93 cultures showed a marked reduction in the level of cleaved caspase-3 (WT-NMDA : 100, Average of WT-NMDA+APV: $5.80 \pm 1.95$, PSD-93KO-NMDA: $28.10 \pm 3.23$, PSD93KO-NMDA+APV: $3.92 \pm 0.61, \mathrm{p}<0.05, \mathrm{n}=3$ ) (Figure 14B).

Thus, this experiment proved that in absence of PSD-93 protein, there is a lower level of NMDA triggered neurotoxicity in cortical cells. This also followed the previous findings where the surface levels of NMDA receptors were reduced in PSD-93KO cortical neurons (Figure 9). Hence, lower surface levels of NMDA receptors in PSD93KO might lead to lower mortality upon activation of these receptors, as well.

\subsubsection{Over-expression of selected PSD-93 isoform can rescue the pro-apoptotic effect of PSD-93}

Since deletion of PSD-93 caused a significant reduction in the levels of cleaved casp3 and hence mortality of neuronal cultures were reduced, I wanted to examine, whether the over-expression of PSD-93 may rescue the neurotoxic phenotype in cortical culture. PSD-93 has six different isoforms. I over-expressed these PSD-93 isoforms against PSD-93 knockout culture background, to check if one or more of these isoforms are sufficient for rescuing wild-type levels of cleaved casp-3.

For this experiment, I used P0 wild-type or PSD-93 knockout cortical cultures. On DIV 5, I transfected the PSD-93KO cultures with lentivirus constructs over- 
Results $\mathrm{P}$ a g e | 64

expression either of $\alpha 1, \alpha 2, \beta, \gamma, \delta, \varepsilon$ isoform of PSD-93 (Krüger et al., 2013a). Each of these constructs, except the $\gamma$ and the $\delta$ constructs, showed mild to dim level of fluorescence. The cells were harvested for western blotting on DIV 16 and expression of PSD-93 isoforms were analyzed by western blotting. 
A
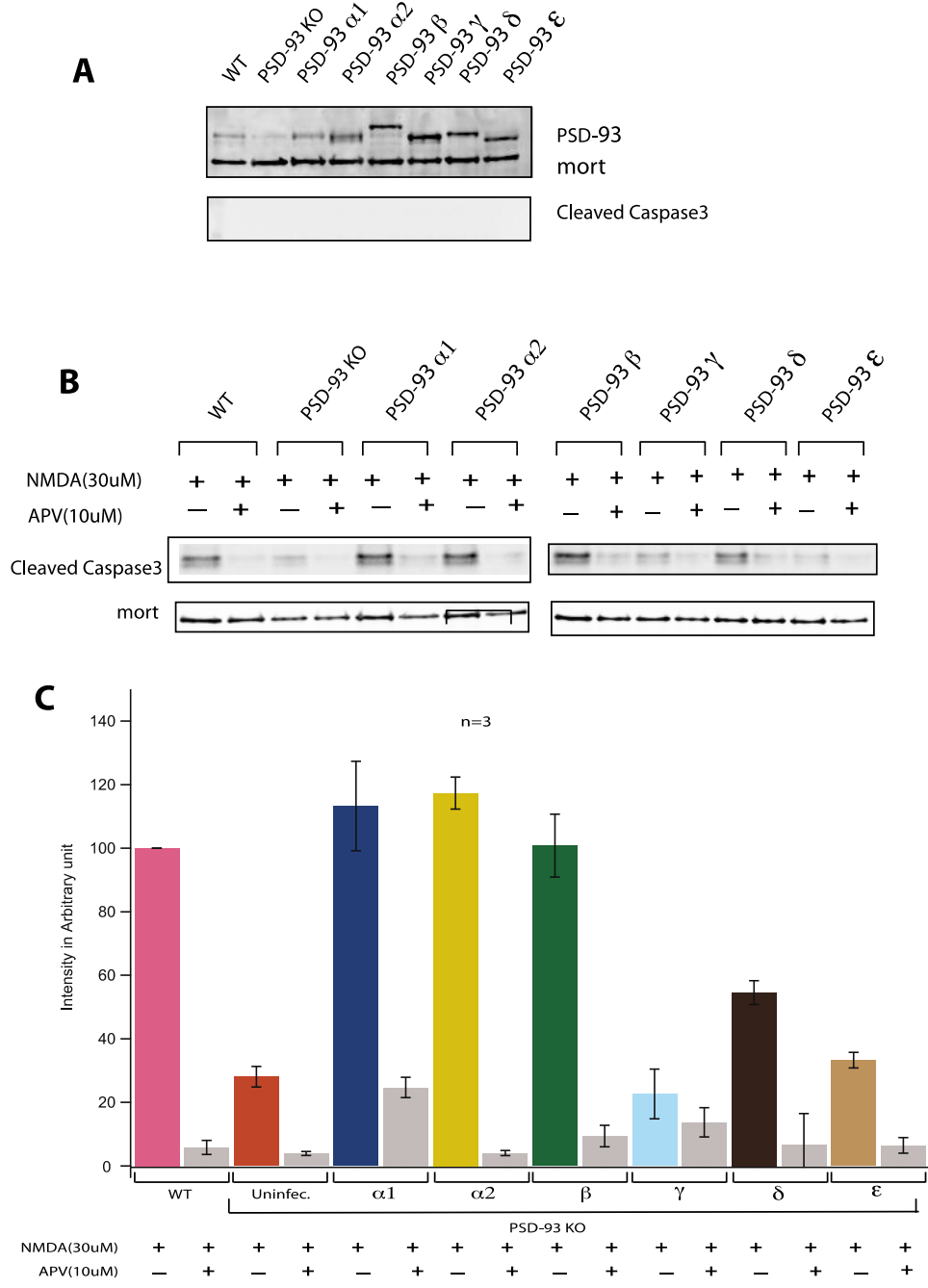

Figure 15: Over-expression of selected PSD-93 isoform rescues NMDA mediated neurotoxicity.

A- Representative western blot showing the expression profile of different PSD-93 over-expression constructs (top). The first lane corresponds to the WT sample, second lane to the PSD-93KO sample, third till the eighth lane corresponds to the six different PSD-93 isoforms $(\alpha 1, \alpha 2, \beta, \gamma, \delta, \varepsilon)$ expressed against the PSD-93KO background respectively. Cleaved casp-3 shows no detectable signal (bottom). B- Representative western blot showing expression of cleaved casp-3 upon NMDA challenge in WT vs. PSD-93KO vs. PSD-93 isoforms over-expression system (top). Mortalin shows no significant change across different samples (bottom). C- Quantification of the level of cleaved casp-3 expressed upon NMDA challenge. Amount of cleaved casp-3 in WT samples (shown in pink) was reduced in PSD-93KO (shown in orange). Over-expression of PSD-9 $\alpha 1$ (shown in navy blue), PSD-93 $\alpha 2$ (shown in yellow) and PSD-93 $\beta$ (shown in green) could completely rescue the level of cleaved casp-3. Over expression of PSD-93 (shown in brown) could only partially rescue the level of cleaved casp-3. Over-expression of PSD-93 $\gamma$ (shown in pale blue) and PSD-93e (shown in pale brown) failed to rescue the cleaved casp-3 level completely. The cleaved caspase- 3 levels could be blocked across all conditions when NMDA receptors were blocked by APV (shown in gray). 
Results $\mathrm{P}$ a g e | 66

I checked for the expression profile of PSD-93. The wild-type lane showed a normal level of the different PSD-93 isoforms, as is expressed in a cortical cell. The PSD-93 knockout lane showed an absence for the PSD-93 bands. The PSD-93 $\alpha 1$ and PSD-93 $\alpha 2$ shows relatively lower expression levels but still it was comparable to wild-type levels of PSD-93. The PSD-93 $\beta$, PSD-93 $\gamma$, PSD-93 $\delta$ and PSD-93 $\varepsilon$ also showed a robust expression of the respective isoforms. The isoforms were identified by their slightly varying molecular weights (Figure 15A (top)). Mortalin was equally distributed over all the samples (bottom).

I also checked for the levels of cleaved casp-3 in these cultures, to rule out any neuronal mortality because of the lenti viral transfection protocol. Cleaved casp-3 gave no detectable signal, indicating, that there was no significant cell death caused by the transfection protocol (Figure 15A (bottom)). To ensure that the cleaved casp-3 antibody worked for this western, this blot was processed in parallel with the cleaved casp-3 blot when PSD-93 isoform over-expression rescued the cleaved casp-3 level (Figure 15B (top)).

I performed the NMDA challenge experiment on DIV 17 on these cultures and analyzed the level of cleaved casp-3 by western blotting.

I looked at the expression of cleaved casp-3 following the NMDA challenge experiment. It could be seen that the level of cleaved casp-3 in the NMDA treated WT sample lane was significantly reduced in the PSD-93KO samples. The NMDA treated on PSD-93KO cultures over-expressing one of PSD-93 $\alpha 1, \alpha 2$ and $\beta$ isoform, was able to rescue the level of cleaved casp-3 to wild-type levels. The $\delta$ isoform could only partially rescue the levels. The PSD-93 $\gamma$ and $\varepsilon$ isoforms failed to rescue the level of cleaved casp-3 (Figure 15B (top)). Mortalin didn't show any significant changes across the samples (Figure 15B (bottom)).

I quantified the level cleaved casp-3 expression in this rescue experiment. Because of difference in level of protein across different blots, the intensities of each band was normalized against its corresponding WT samples treated with NMDA and then expressed as a percentage. The wild-type sample hence, has no variance as its always 
$100 \%$ and every other band are in relative percentage to it (WT-NMDA: $100 \pm 0, \mathrm{Av}-$ erage WT-NMDA+APV: $5.80 \pm 1.95$, PSD-93KO-NMDA: $28.10 \pm 3.23$, PSD-93KO-

NMDA+APV: $3.92 \pm 0.61$, PSD-93 $\alpha 1$-NMDA: $113.28 \pm 14.08$, PSD-93 $\alpha 1-$

NMDA+APV: $24.71 \pm 3.19$, PSD-93 $\alpha 2-N M D A: 117.34 \pm 5.02$, PSD-93 $\alpha 2-$

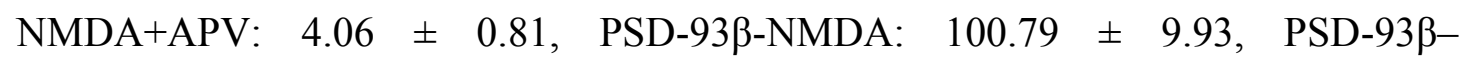
NMDA+APV: $9.40 \pm 3.39$, PSD-93 $\gamma-N M D A: 22.67 \pm 7.85$, PSD-93 $\gamma-N M D A+A P V:$ $13.70 \pm 4.51$, PSD-93 $\delta$-NMDA: $54.54 \pm 17.98$, PSD-93 $\delta$-NMDA+APV: $6.74 \pm 3.76$, PSD-93 - -NMDA: $33.29 \pm 9.73$, PSD-93 - NMDA+APV: $6.46 \pm 2.47, n=3$ ) (Figure $15 \mathrm{C})$.

This experiment showed that whereas down-regulation of PSD-93 causes reduced neurotoxicity in cortical cultures, over-expression of selected isoforms of PSD-93 $(\alpha 1, \alpha 2, \beta)$ was capable of rescuing the neurotoxic phenotype. The PSD-93 $\delta$ isoform may be partially capable of rescuing and may need other isoforms for a full rescue. It also showed that the PSD-93 $\gamma$ and PSD-93 $\varepsilon$ failed to rescue the neurotoxicity completely.

\subsubsection{GluN2B is the major contributing NMDA receptor subunit for the NMDA mediated neurotoxicity}

Reports claim that GluN2A containing NMDA receptors tend to localize more in the synaptic zone (Stocca and Vicini, 1998; Tovar and Westbrook, 1999) and are pro-cell survival(Liu et al., 2007) whereas GluN2B containing NMDA receptors are more extra-synaptic and are pro-apoptotic in nature (Wang et al., 2013). Since PSD-93KO showed surface reduction in both GluN2A and GluN2B receptors but to varying degrees, I wanted to estimate the percentage contribution of these receptors in our NMDA challenge experiments.

For this purpose, I wanted to block either the GluN2A or the GluN2B receptors using specific pharmacological antagonist and look for their respective contribution in NMDA mediated neurotoxicity. I used wild-type cortical cultures at DIV 17 and performed our NMDA challenge experiments. I used $30 \mu \mathrm{M}$ of $\mathrm{Zn}^{2+}$ for blocking GluN2A receptors, and $10 \mu \mathrm{M}$ of Ifenprodil to block the GluN2B receptors. I used 10 
Results $\mathrm{P}$ a g e $\mid 68$

mM APV to block all NMDA receptor subtypes. $10 \mu \mathrm{M}$ NBQX was used in all reactions to prevent any AMPA transmission.

A

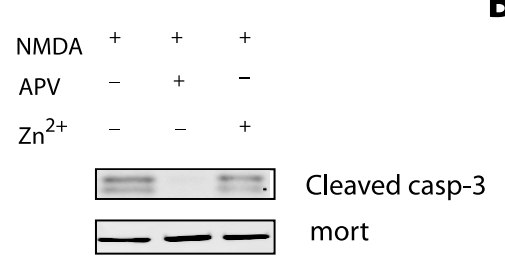

B

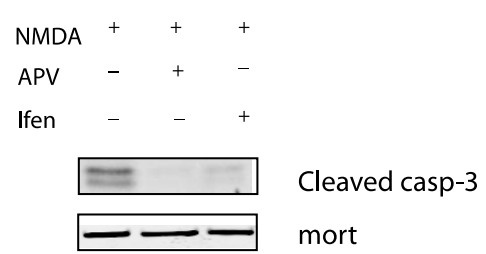

C

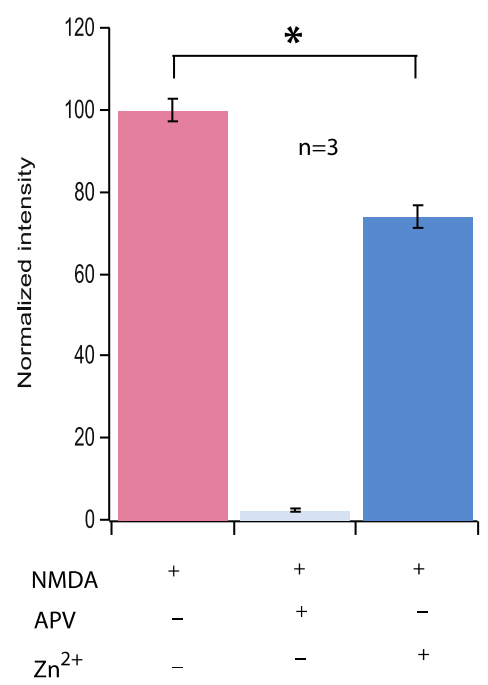

D

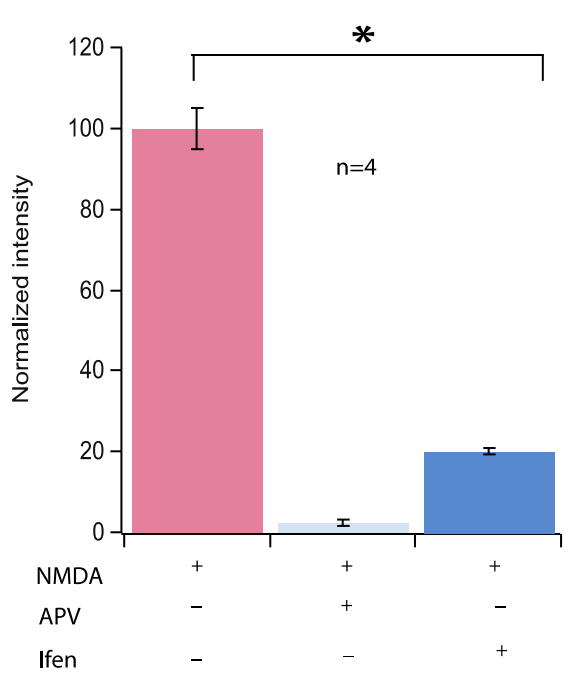

Figure 16: GluN2A vs. GluN2B contribution in NMDA mediated neurotoxicity. A- Representative western blot showing the level of cleaved casp-3 (top) in WT cultures treated with NMDA (30 $\mu \mathrm{M})$ either in presence or absence of APV and $\mathrm{Zn}^{2+}$. Mortalin stays equally distributed in all samples (bottom). B- Representative western blot showing the level of cleaved casp-3 (top) in WT cultures treated with NMDA (30 $\mu \mathrm{M})$ either in presence or absence of APV and ifenprodil. Mortalin stays equally distributed in all samples (bottom). C- Quantification of cleaved casp-3 normalized against the average WT NMDA treated band. There was small but significant change in the level of cleaved casp-3 from the WT NMDA treated (shown in pink) to the WT NMDA treated blocked with $\mathrm{Zn}^{2+}$ (shown in blue ), $\mathrm{p}<0.05$, T-test. D- Quantification of cleaved casp-3 normalized against the average WT NMDA treated band. There was a significant change in the level of cleaved casp-3 from the WT NMDA treated (shown in pink) to the WT NMDA treated blocked with ifenprodil (shown in blue), $\mathrm{p}<0.05, \mathrm{n}=3$, T-test. APV treatment (shown in pale gray ) blocks the level of cleaved casp-3 in both C \& $\mathbf{D}$.

Representative western blot image shows the effect of $\mathrm{Zn}^{2+}$ mediated blockade of GluN2A receptor on NMDA mediated neurotoxicity (Figure 16A). The first lane shows the level of cleaved casp-3 upon NMDA challenge when no NMDA receptor antagonists were used. The second lane shows that the level of cleaved casp-3 upon 
NMDA treatment could almost completely be blocked upon using APV, which blocks all NMDA receptor subtypes. The third lane shows the amount of cleaved casp-3 when $\mathrm{Zn}^{2+}$ blocks the GluN2A subunits

Upon quantification, I see that blocking the GluN2A subunit could reduce almost 25 $\%$ of the effect of NMDA neurotoxicity. The level of mortalin, used as a loading control, didn't change between samples. I normalized all band intensities to the average band intensity from the NMDA treated lane and expressed it as a percentage (Average intensity NMDA treated: $100 \pm 2.38, \mathrm{NMDA}+\mathrm{APV}: 2.45 \pm 0.26, \mathrm{NMDA}+\mathrm{Zn}^{2+}$ : $73.89 \pm 2.31, \mathrm{n}=3, \mathrm{p}<0.05$ ) (Figure 16C).

Representative western blot image shows the effect of ifenprodil mediated blockade of GluN2A receptor on NMDA mediated neurotoxicity (Figure 16B). The first lane shows the level of cleaved casp-3 upon NMDA challenge when no NMDA receptor antagonists were used. The second lane shows that the level of cleaved casp-3 upon NMDA challenge could almost completely be blocked by APV. The third lane shows the amount of cleaved casp-3 when only the GluN2B subunits are blocked by ifenprodil.

Blocking the GluN2B subunit by ifenprodil could reduce $80 \%$ effect of NMDA mediated neurotoxicity. Mortalin remained unchanged across different lanes. I normalized all band intensities to the average band intensity from the NMDA treated lane and then expressed it as a percentage (Average intensity NMDA treated: $100 \pm 5.16$, $\mathrm{NMDA}+\mathrm{APV}: 2.22 \pm 0.83, \mathrm{NMDA}+$ Ifen $: 20.24 \pm 0.81, \mathrm{n}=4, \mathrm{p}<0.05)$ (Figure 16D).

Thus, this experiments showed that the principle subunit, which is responsible for the NMDA mediated neurotoxicity was the GluN2B subunit as blocking it could prevent more than $80 \%$ of NMDA neurotoxicity. It also fits to the current understanding of NMDA receptor mediated neurotoxicity where GluN2B receptors are thought to be more pro-apoptotic whereas GluN2A are more pro-cell survival (Wang et al., 2013, Liu et al., 2007). $\mathrm{Zn}^{2+}$ is a blocker for both GluN2A and GluN2B, but it is supposed to be specific for GluN2A over $30 \mu \mathrm{M}$ concentration, below which it can also block 
Results $\mathrm{P}$ a g e $\mid 70$

GluN2B. So, the $30 \mu \mathrm{M}$ concentration of $\mathrm{Zn}^{2+}$, which I had used should be specific for GluN2A only. However, a partial blockade of a small percentage of GluN2B receptors cannot be ruled out. In that case, the effect of GluN2A on the process of NMDA neurotoxicity would be even lower.

\subsubsection{P38 MAPK activation profile during NMDA neurotoxicity challenge experi- ment}

Several studies have shown that primarily the P38MAPK pathway is responsible for mediating the NMDA mediated neurotoxicity (Barone et al., 2001). So I wanted to check, if the neurotoxic activity of the GluN2B subunit was mediated by activation of P38.

For this purpose, I checked for the activation profile of P38 MAPK in WT cortical neurons when challenged with NMDA. Since P38 MAPK is one of the early molecules in the signaling cascade that activates the pro-apoptotic pathway in neurons, I chose time points of $10 \mathrm{~min}, 20 \mathrm{~min}, 30 \mathrm{~min}$ and 1 hour of treatment with NMDA and checked for the levels of active P38.

To check if the level of P38 activation is transient or is maintained continually after NMDA challenge, I also measured the level of active P38, for each of the aforementioned time points, after $24 \mathrm{~h}$ incubation. 


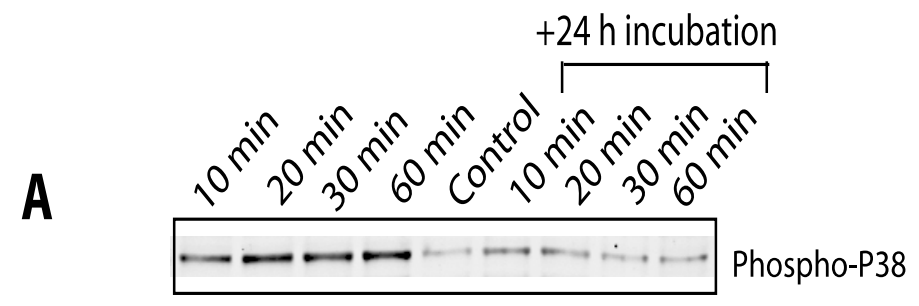

B Treatment

Treatment $+24 \mathrm{~h}$ incubation

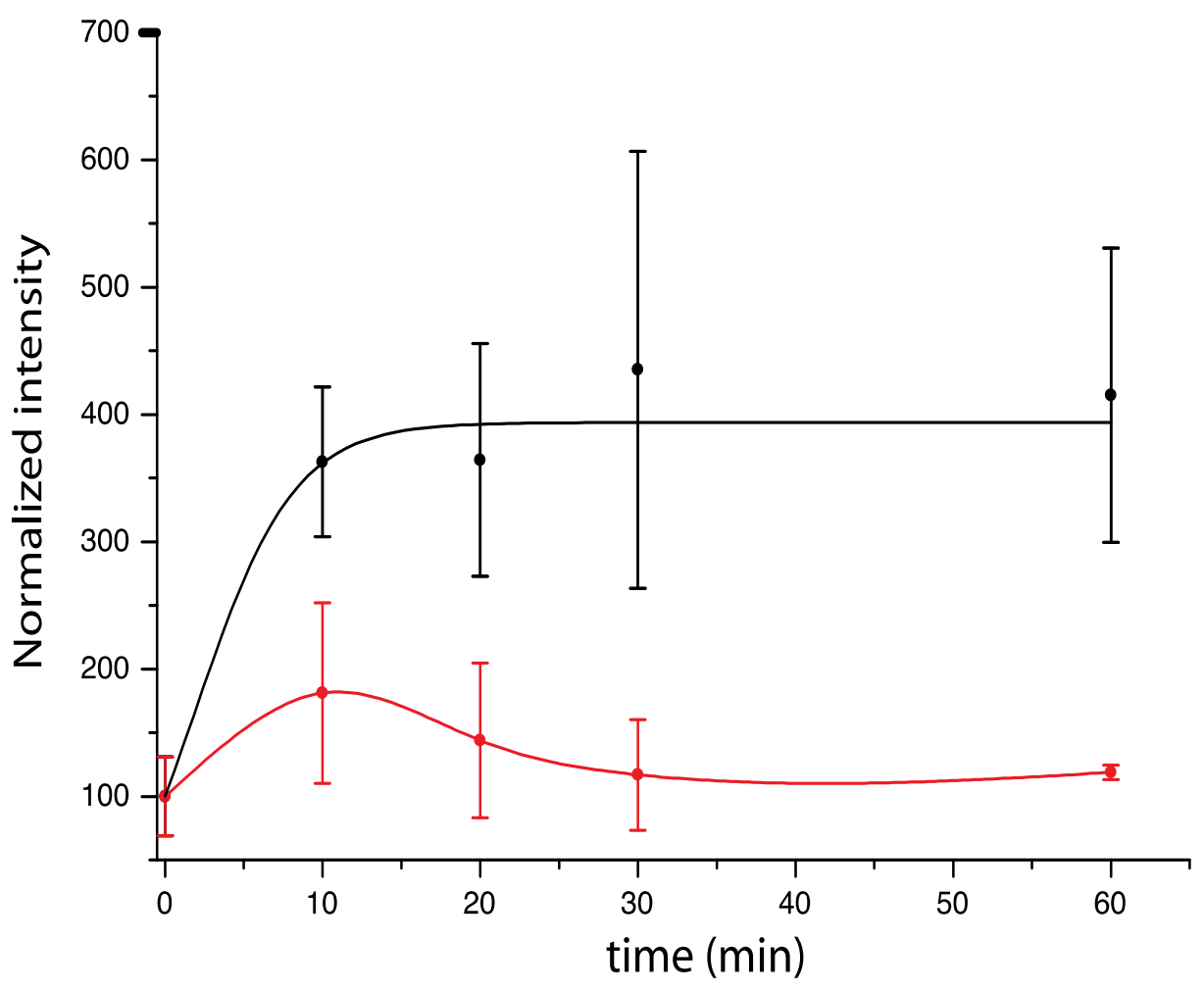

Figure 17: P38 MAPK activation curve during NMDA challenge experiment. A- Representative western blot showing the level of active P38 MAPK in WT cultures treated with NMDA $(30 \mu \mathrm{M})$ for different time points. B- Quantification of time vs. concentration of active P38 (normalized against the 1 hour time point concentration). The level of active P38 steadily increases from 10 min of NMDA treatment and reaches saturation after 30 min of NMDA treatment (black solid trend line) but comes down to control level post 24 incubation in fresh media (red solid trend line). The distribution can be fit to a exponential growth curve (black dotted line).

The level of active P38 seems to increase from the control level following NMDA treatment right from 10 min of incubation. This increase in active P38 MAPK level 
Results $\mathrm{P}$ a g e $\mid 72$

however drops close to base line when incubated in fresh media for 24 hours, post NMDA treatment (Figure 17A).

I quantified the activation profile of P38MAPK upon NMDA challenge. For quantification, all band intensities were normalized against the control untreated band average intensity and expressed as a percentage. The level of active P38 kept increasing but reached saturation after 30 min of NMDA treatment (Average normalized intensity for $30 \mu \mathrm{M}$ NMDA treatment for control: $99.99 \pm 31.05,10 \mathrm{~min}: 362.7 \pm 58.74,20$ min: $364.32 \pm 91.42,30$ min: $435.13 \pm 171.69,1$ hour: $415.13 \pm 115.68, n=3)$. After 24 hours of incubation in fresh media however, the level of active P38 goes back to control level (Average normalized intensity for $30 \mu \mathrm{M}$ NMDA treatment for control: $99.99 \pm 31.05,10 \mathrm{~min}: 181.08 \pm 70.83,20 \mathrm{~min}: 143.78 \pm 60.86,30 \mathrm{~min}: 116.75 \pm$ 43.40, 1 hour: $118.91 \pm 5.64, \mathrm{n}=3$ ). The dose-response data can be fit into an exponential growth curve (Figure 17B).

This showed that NMDA neurotoxicity via GluN2B does up-regulates the P38 MAPK which in turn may activate the downstream pro-apoptotic signaling cascade.

\subsubsection{PSD-93 show reduced levels of active P38 during NMDA challenge}

Our earlier results have shown a reduced surface GluN2B receptor in PSD-93KO neurons. We also showed that PSD-93KO cultures also have reduced level of active caspase-3. Since we also confirmed in the previous experiment that NMDA challenge increases the level of active P38MAPK, we checked whether the level of active P38 is also reduced in PSD-93KO neurons.

I performed 1 hour of $30 \mu \mathrm{M}$ NMDA treatment on both WT and PSD-93 KO cultures in presence and in absence of APV and checked for the level of activated P38 immediately after the NMDA treatment. Mortalin was used as a loading control. 


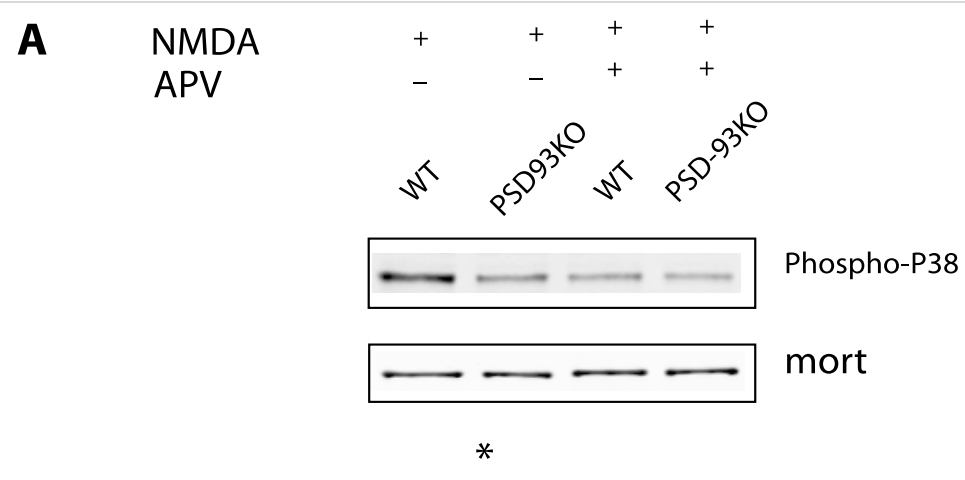

B

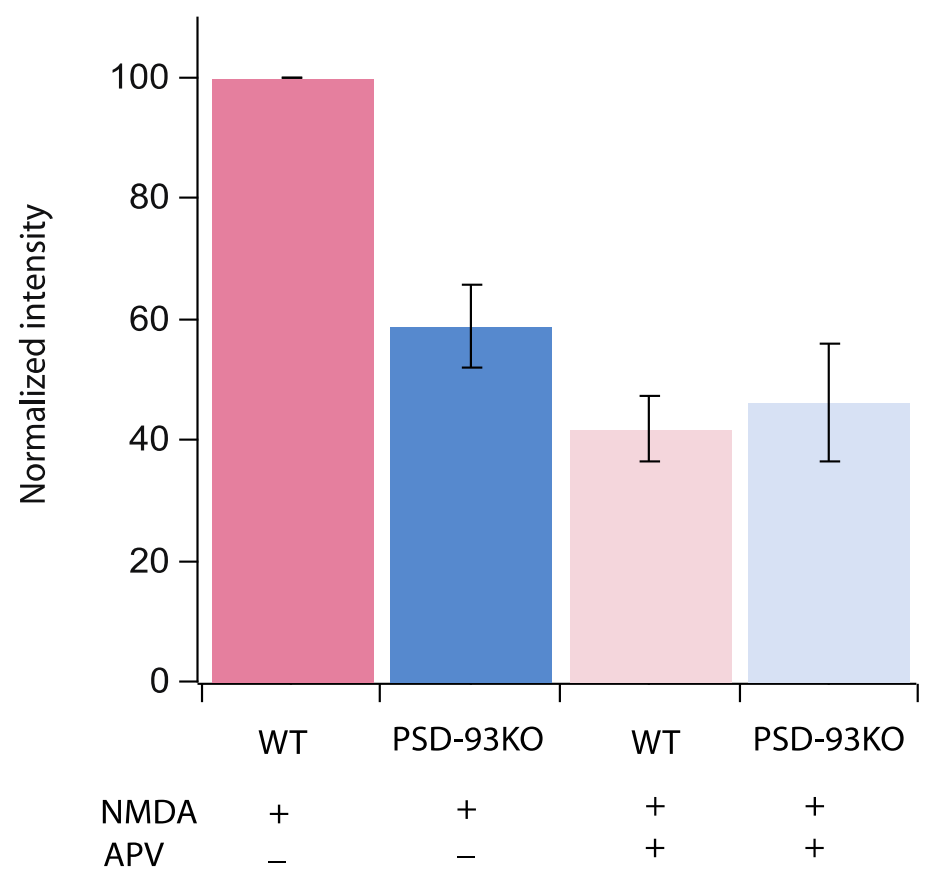

Figure 18: PSD-93KO has reduced level of active P38 MAPK during NMDA challenge. A-(top) Representative western blot showing the active P38 level in wild-type vs. PSD-93KO culture in presence or in absence of APV (top). PSD-93KO could block rise in active P38 levels almost completely. APV also blocks the rise in active P38 in both WT as well as PSD-93KO cultures. Mortalin remains equally distributed across all samples (bottom). B- Quantification of the level of active P38 normalized against respective WT NMDA treated controls. The WT active P38 level (shown in pink) was reduced in PSD-93KO cultures (shown in blue). APV could block this effect in both WT (shown in pale pink) as well as PSD-93KO cultures (shown in pale blue), $\mathrm{p}<0.05$, TTest.

The representative western blot image shows the level of active P38 in cultures treated with NMDA. The first and the second lane corresponds to WT and PSD-93KO cultures treated with NMDA and the third and fourth lane corresponds to WT and PSD-93 cultures treated with NMDA in presence of APV (Figure 18A). 
Results $\mathrm{P}$ a g e $\mid 74$

For quantification of active P38 MAPK levels, I normalized all band intensities to their corresponding WT NMDA treated value. Thus, when expressed as a percentage, the wild-type showed no variance while all the rest showed levels relative to it. The PSD-93KO cultures showed a significant reduction in the level of active P38 upon NMDA challenge from its WT counterpart. Blocking the NMDA receptor activation by APV in both cases could prevent the activation of P38 (Average normalized P38 intensity for WT NMDA: 100, PSD-93KO NMDA: $58.82 \pm 6.81$, WT NMDA+APV: $41.72 \pm 5.48$, PSD-93KO NMDA+APV: $46.19 \pm 9.84, \mathrm{n}=3, \mathrm{p}<0.05$, T-test) (Figure 18B).

\subsubsection{PSD-93KO cultures have lower level of active P38 due of higher level of ac- tive STEP}

A Striatal Enriched Protein Phosphatase or STEP is known to causes dephosphorylation of P38 MAPK, thus keeping it inactive in basal conditions. Previous studies had shown that during NMDA challenge, GluN2B mediated influx of $\mathrm{Ca}^{2+}$, causes activation of $\mathrm{m}$-calpain, which is a $\mathrm{Ca}^{2+}$ - dependent protease. Active $\mathrm{m}$-calpain leads degradation of active STEP (Poddar et al., 2010). Degradation of STEP causes secondary activation in active P38 MAPK and hence promotes the downstream apoptotic pathway.

I checked whether in PSD-93KO animals the level of active STEP is altered. I performed 1 hour of $30 \mu \mathrm{M}$ NMDA treatment on both WT and PSD-93 KO cultures in presence and in absence of APV and checked for the level of non-phospho STEP (active) immediately after the NMDA treatment. 
A

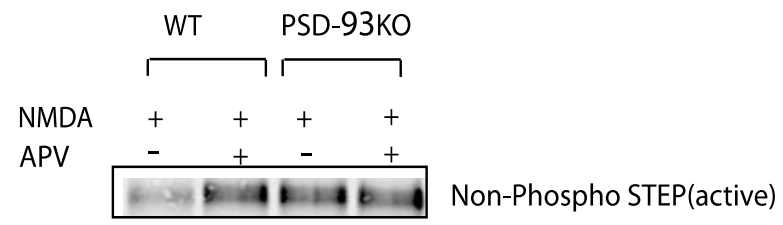

B

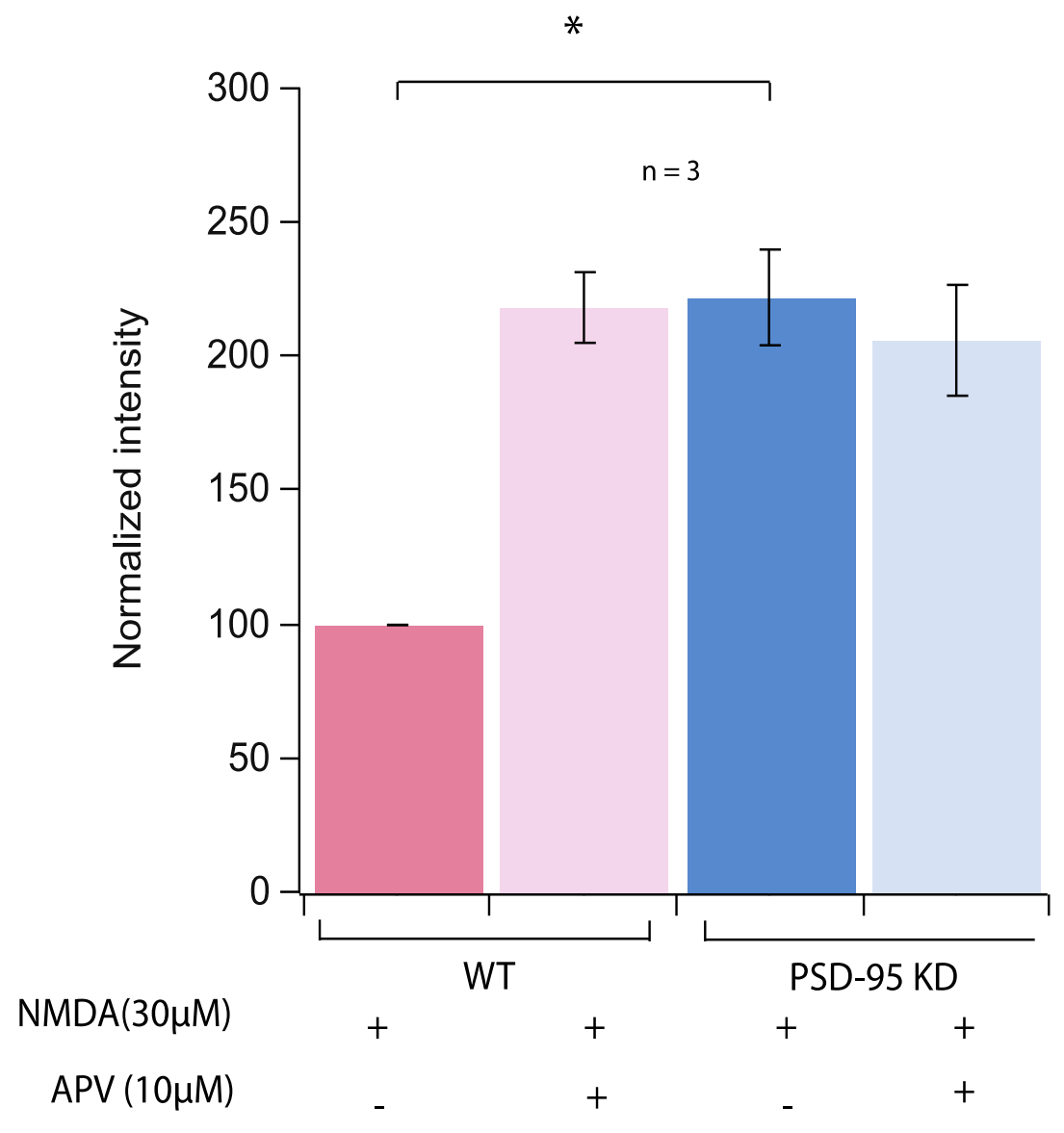

Figure 19: PSD-93KO has elevated levels of active STEP during NMDA challenge. A- Representative western blot showing the active STEP level in wild-type vs. PSD-93KO culture in presence or in absence of APV. PSD93KO could block the reduction of active STEP. APV also blocks the reduction in active STEP in both WT as well as in PSD-93KO cultures. B- Quantification of the level of active STEP normalized against the average WT NMDA treated level. The WT NMDA treated sample (shown in pink) had reduced level of active STEP than in PSD-93KO cultures (shown in blue). APV treatment kept the level of active STEP to an elevated level in both WT as well as PSD-93KO cultures (shown in pale blue), $\mathrm{p}<0.05$, T-test.

Representative western blot showing the level of active STEP in cultures treated with NMDA (Figure 19A). The first and the third lane corresponds to WT and PSD-93KO cultures treated with NMDA and the second and fourth lane corresponds to WT and PSD-93 cultures treated with NMDA in presence of APV. 
I quantified the level of active STEP between wild-type and PSD-93KO cultures on being treated with NMDA (Figure 19B). For quantification of active STEP, I normalized all band intensities to the average WT NMDA treated lane and expressed it as percentage. Wild-type NMDA treated cultures showed reduction in the level of active STEP but PSD-93KO cultures showed a significantly elevated level of active STEP post NMDA treatment. Blocking the NMDA receptor activation by APV in both cases could keep the level of active STEP higher (Average normalized active STEP intensity for WT NMDA: $100 \pm 0.42$, WT NMDA+APV: $218.50 \pm 12.83$, PSD-93KO NMDA: $221.59 \pm 18.03$, PSD-93KO NMDA+APV: $205.84 \pm 21.02, \mathrm{n}=3, \mathrm{p}<0.05$, T-test).

This experiment showed that following a NMDA challenge, when NMDA receptors are activated in WT neurons, it causes a reduction of the active STEP. This in turn helps to elevate the levels of active P38 MAPK, which can then trigger the apoptotic pathways in the cell. In PSD-93 due to reduced surface NMDA receptors (Figure 9), the active STEP remains higher even after NMDA exposure. This leads to lower level of active P38 MAPK and hence reduced level of apoptosis in these cells.

\subsection{Comparing the neuro-protection due to the loss of PSD-93 and PSD-95 against NMDA mediated neurotoxicity in cor- tical neurons}

\subsubsection{PSD-95 deletion also causes a reduction in cortical culture mortality but to a lesser extent than PSD-93 deletion}

PSD-95 had been shown to be involved in interacting with c-terminal region of NMDA receptors via their PDZ 1/2 domain and promote their synaptic clustering by preventing GluN2B mediated internalization (Roche et al., 2001). Previous studies had been performed using anti-sense oligo-nucleotides against PSD-95 to show that absence of PSD-95 protected neurons against NMDA mediated excito-toxicity (Sattler, 1999a) and leads to enhanced cell survival in post-ischemic hippocampus. Neuronal NO had been thought to cause glutamate induced neurotoxicity in neurons 
Results $\mathrm{P}$ a g e $\mid 77$

and it was found out that PSD-95 helps connect NMDAR mediated $\mathrm{Ca}^{2+}$-influx with NO production and hence promotes neurotoxicity (Sattler, 1999a). So I wanted to check whether deletion of PSD-95 has an effect on NMDA mediated neurotoxicity on cortical neurons.

To achieve this, I used the lentiviral vector based constructs FRUGW, which contains RNAi against endogenous PSD-95 protein (Schlüter et al., 2006). Wild-type cultures were infected on DIV 7 with the lenti virus carrying the PSD-95 knockdown construct. The transfection efficiency and infectivity were monitored using the GFP fluorescence. Cultures were harvested on DIV 14 to check the knockdown efficiency of the FRUGW construct. 
A

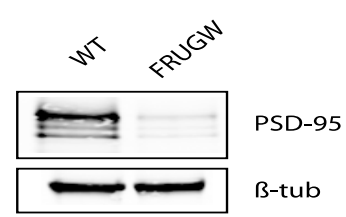

B

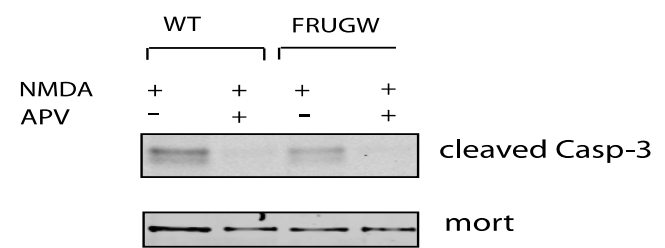

C

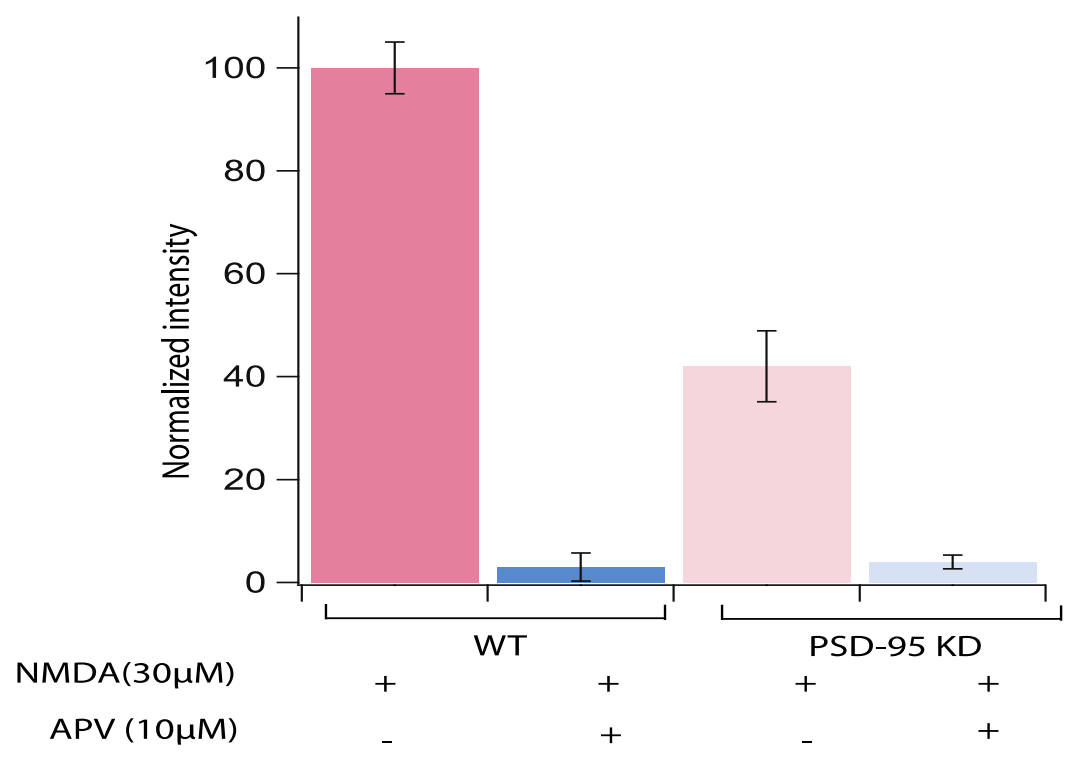

Figure 20: Knockdown of PSD-95 causes reduction in NMDA mediated neurotoxicity. A- Western blot showing efficiency of the FRUGW virus to knockdown PSD-95 in culture. The blot shows more than $95 \%$ reduction in levels of PSD-95 (top). $\beta$-tubulin shows equal intensity in both lanes (bottom). B- Representative western blot showing the cleaved casp-3 level in wild-type vs. PSD-95KD culture in presence or in absence of APV (top). APV could block the cleaved casp-3 levels almost completely both in WT as well as PSD-95KD cultures. Mortalin remains equally distributed across all samples (bottom). C- Quantification of the level of cleaved casp-3 normalized against the average of the WT NMDA treated sample. The WT cleaved casp-3 level (shown in pink) was reduced in PSD-95KD cultures (shown in light pink) ( $p<0.05$, n.s., $n=3$, T-Test). APV could block this effect in both WT (shown in blue) as well as PSD-95KD cultures (shown in light blue).

More than $95 \%$ of knockdown for the PSD-95 protein as compared to the wild-type uninfected control (Figure 20A (top)). $\beta$-tubulin was used as a protein loading control (Figure 20 (Bottom)). 
Results $\mathrm{P}$ a g e $\mid 79$

I performed a NMDA challenge on both wild-type uninfected as well as the PSD-95 knockdown cultures. After 24 hours incubation, the cells were harvested and quantified by western blot for the levels of cleaved caspase-3 protein.

I could see a reduction in cleaved casp-3 levels in the PSD-95 knockdown cultures (Figure 20B). Mortalin levels remained unaffected across samples.

The level of cleaved casp-3 in uninfected vs. PSD-95 KD cultures were quantified (Figure 20C). The band intensities in all lanes, from independent experiments, were all normalized to the average intensity of the uninfected WT NMDA treated culture and plotted as a percentage (WT-NMDA: $100 \pm 5.04$, WT-NMDA+APV: $2.75 \pm 2.75$, PSD-95KD-NMDA: $41.74 \pm 6.88$, PSD-95KD-NMDA+APV: $3.67 \pm 0.91, \mathrm{p}<0.05$ $\mathrm{n}=3)$.

Thus, the down-regulation of PSD-95 can cause reduction in cleaved casp-3 levels in culture. Previous results indicate that PSD-93 was capable of reducing the level of cleaved casp-3 almost to the same or slightly higher degree. This may indicate that PSD-93, in spite of being a relatively low abundance MAGUK has a crucial role to play when it comes to NMDA mediated pro-apoptotic signaling. 


\subsubsection{Deletion of PSD-93 has a more severe effect on NMDA mediated neurotoxi- city than deletion of PSD-95}

In the previous experiment I saw, that PSD-93 showed a greater reduction in cortical culture mortality than PSD-95, when challenged with $30 \mu \mathrm{M}$ of NMDA. Next I wanted to see if at elevated doses of NMDA challenge, both PSD-93 and PSD-95 deletion could still be neuro-protective in nature.

For this experiment, we used wild-type cortical cultures and PSD-93 knockout cortical cultures. For knocking down PSD-95, I transfected on DIV 5, wild-type cortical cultures with the FRUGW construct. On DIV 17 of these cultures, I performed the NMDA challenge experiment on all three cultures.

For the wild-type cultures I used a standard dose of $30 \mu \mathrm{M}$ NMDA, as it had already been shown that $30 \mu \mathrm{M}$ of NMDA is a saturating concentration for wild-type cortical neurons (Figure 12). For the PSD-93 knockout cultures and for the PSD-95 knock down cultures, I used doses of $30 \mu \mathrm{M}, 50 \mu \mathrm{M}$ and $100 \mu \mathrm{M}$ NMDA. Cultures were harvested 24 hours after the protocol and analyzed by western blot for the levels of cleaved casp-3. 
A
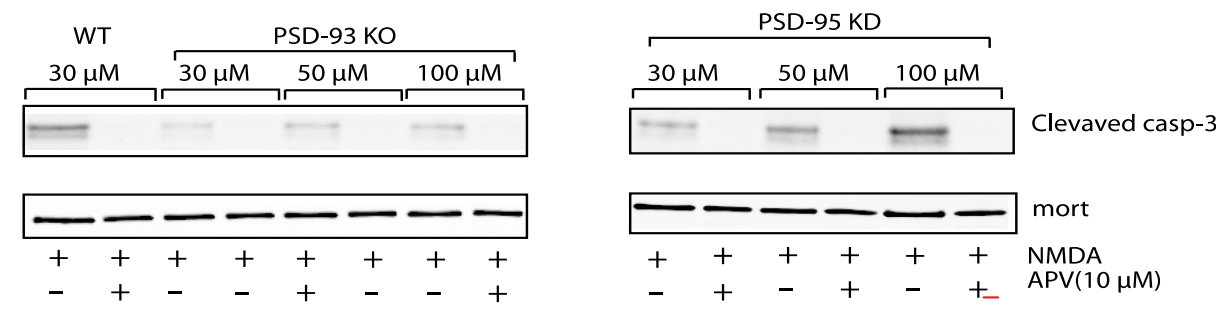

B

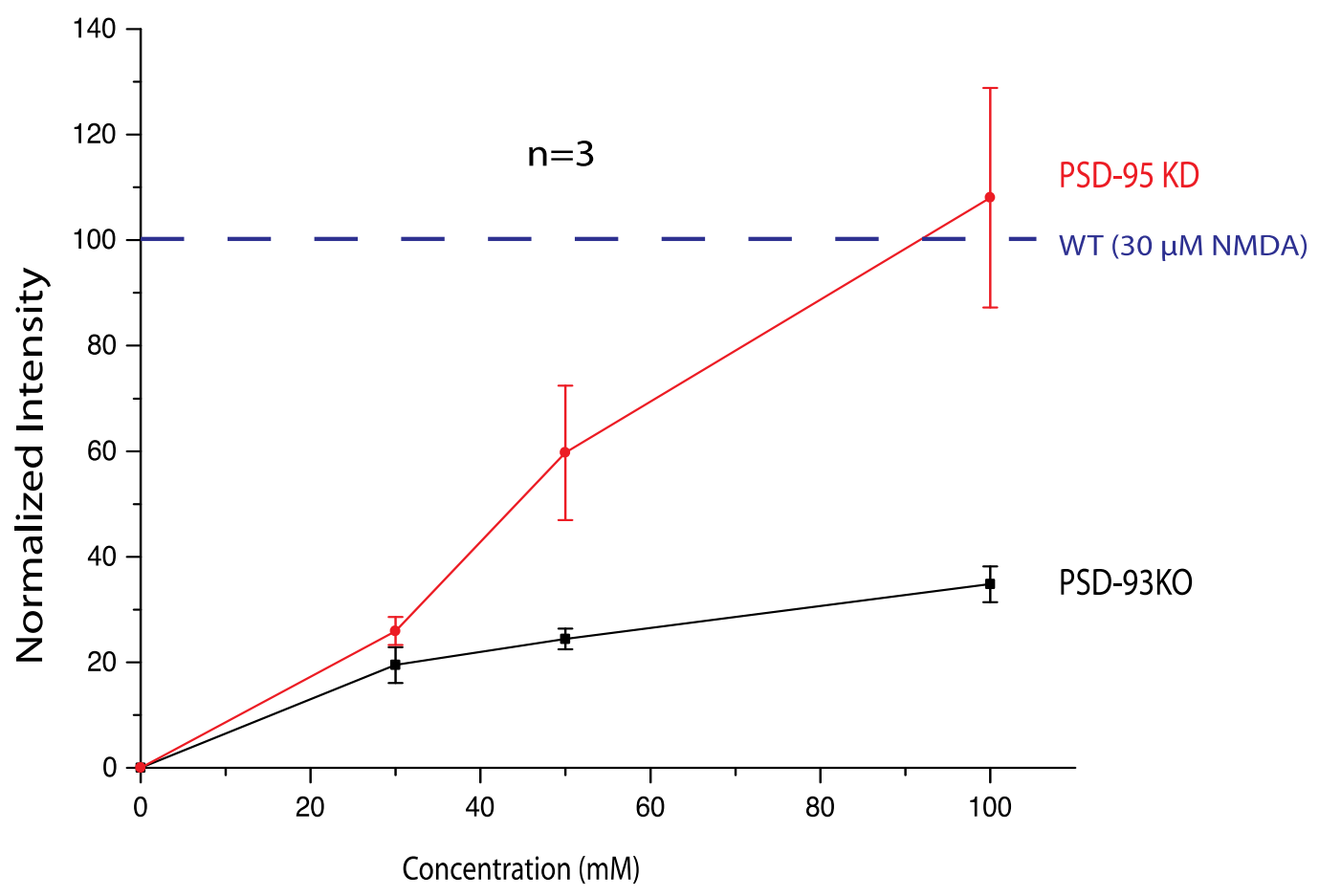

Figure 21: Deletion of PSD-93 provides better protection against NMDA induced neurotoxicity

than deletion of PSD-95. A- Representative western blot showing the cleaved casp-3 level in wild-type vs. PSD-93KO vs. PSD-95KD culture in presence or in absence of APV (top). WT cultures treated with $30 \mu \mathrm{M}$ NMDA. Both PSD-93KO cultures and PSD-95KD cultures were treated with three different NMDA concentrations of $30 \mu \mathrm{M}, 50 \mu \mathrm{M}$ and $100 \mu \mathrm{M}$ NMDA. APV could block the level of cleaved casp-3 completely in WT, PSD-93KO and PSD-95KD samples. Mortalin remains equally distributed across all samples (bottom). B- Quantification of the level of cleaved casp-3 normalized against the average of the WT NMDA treated sample. The WT cleaved casp-3 level (shown in blue dotted line) was measured as 100 percent. The PSD-93KO samples treated with $30 \mu \mathrm{M}, 50 \mu \mathrm{M}$ and $100 \mu \mathrm{M}$ NMDA are shown by the black dots and joined by the black trend line. The level of cleaved casp-3 in the PSD-95KD samples treated with $30 \mu \mathrm{M}, 50 \mu \mathrm{M}$ and $100 \mu \mathrm{M}$ NMDA are represented as red dots and joined with the red trend line. The PSD-93KO cultures showed reduced level of cleaved caspase-3 at all three concentrations of NMDA used. But in the PSD-95 knockdown cultures the level of cleaved caspase-3 could be rescued back to wild-type level with increased dose of NMDA treatment. 
The representative western blot shows that the $30 \mu \mathrm{M}$ dose of NMDA challenge cause a significantly reduced level of cleaved casp-3 in the PSD-93KO culture. Even with increasing concentration of NMDA, PSD-93KO failed to rescue the wild-type level of cleaved casp-3. In the PSD-95KD cultures, $30 \mu \mathrm{M}$ dose of NMDA showed a similar reduction in the level of cleaved casp-3, but with increasing concentration of NMDA, the level of cleaved casp-3 was rescued back to wild-type level. The production of cleaved casp-3 was almost completely blocked in presence of $10 \mu \mathrm{M}$ APV. Mortalin, used as a loading control, stayed normal across all samples (Figure 21A).

I quantified the cleaved casp-3 levels by normalizing each band of cleaved casp-3 with their corresponding WT-NMDA band, to account for the differing amount of proteins between different blots. Even at $100 \mu \mathrm{M}$ of NMDA PSD-93KO could only rescue up to $35 \%$ of WT levels of cleaved casp-3, while at $100 \mu \mathrm{M}$ of NMDA, PSD95KD could rescue the WT level of cleaved casp-3 completely. The band intensities of all reaction done in presence of APV was too weak to quantify and hence was not plotted. (Average intensity of WT-NMDA: $100 \pm 0$, PSD-93KO-30 $\mu \mathrm{M}$ NMDA: $19.46 \pm 3.40$, PSD-93KO-50 $\mu \mathrm{M}$ NMDA: $24.43 \pm 1.95$, PSD-93KO-100 $\mu \mathrm{M}$ NMDA: $34.79 \pm 3.38$, PSD-95KD-30 $\mu$ M NMDA: $26.72 \pm 3.56$, PSD-95KD-50 $\mu \mathrm{M}$ NMDA: $71.95 \pm 4.65$, PSD-95KD-100 $\mu \mathrm{M}$ NMDA: $128.74 \pm 3.42, \mathrm{n}=3$ ) (Figure 21B).

The above experiment indicated, that at lower doses of NMDA challenge, both PSD93 and PSD-95 are neuro-protective in nature. When the dose of neurotoxic NMDA is increased, PSD-95 knockdown can no longer do its neuro-protection, while PSD93knockout continues to be neuro-protective. This showed that in cortex PSD-93 has a more prominent role in NMDA mediated neuro-toxicity. 


\section{Discussion}

\subsection{PSD-93 regulates surface levels of NMDA receptors in a brain region spe- cific manner}

In recent years there has been much debate whether PSD-93 has a role to play in NMDA receptor function in synapses. Some reports suggest that even in absence of PSD-93 there is normal synapse assembly and maturation in cerebellum neurons (McGee et al., 2001a). But in spinal dorsal horn neurons, the surface expressions of NMDA receptors were reduced in PSD-93KO animals (Tao et al., 2003). When it came to effect of PSD-93 in the synaptic NMDA receptor function, the results were contradictory as well. While PSD-93KO animals had normal level of basal synaptic current in hippocampus, RNAi mediated knockdown of PSD-93 caused 50\% reduction in AMPA receptor mediated current but not NMDA receptor mediated current (Elias et al., 2006a). On the contrary, Tao et al (2003) showed that in forebrain, PSD$93 \mathrm{KO}$ caused a reduction in synaptic NMDA receptor current while the AMPA receptor mediated current remained unchanged. While in younger animals (P20-30) There was no change in the AMPA/NMDA ratio in PSD-93KO animals, in older animals (P60-74), PSD-93KO were shown to have reduction, suggesting that PSD-93 also have a time-dependent change is functionality (Krüger et al., 2013b). All this reports point towards one direction that the role of PSD-93 is highly divergent and its function depends on specific brain region and developmental state of animals.

Knowing this, I wanted to see how PSD-93 effects in the neo-cortex and chose the L2/3 of mice visual cortex for investigating the role of PSD-93. Since GluN2A and GluN2B are the two most important subunits of NMDA receptors in cortex, I checked for the levels of both of them. When I looked at the total levels of NMDA receptors, there seemed to be no change between WT and PSD-93KO animals (Figure 5). But the surface expression levels of NMDA receptors, both GluN2A and GluN2B subunits, showed a reduction in the PSD-93KO animals (Figure 4). However, no changes were found in the surface levels of AMPA receptors (Figure 6). The surface level of receptors includes receptors located both in the synapse as well as extra-synapse and the protocol I used measured both this pool of NMDA receptors. It is possible to distin- 
Discussion $\mathrm{P}$ a g e $\mid \mathbf{8 4}$

guish between synaptic and extra-synaptic NMDA receptors by doing a sub-cellular fractionation protocol. Previous reports on cortical level of NMDA receptors had shown no variation in synaptic fraction of PSD-93KO mice (Bonnet et al., 2013). So this would indicate that changes in NMDA receptors that I see is more extra-synaptic in nature.

To identify if PSD-93 and NMDA receptors have any in vivo physical interaction, I checked if PSD-93 co-immunoprecipitates with NMDA receptor subunits. Coimmunoprecipitation protocol uses antibodies to precipitate one protein from a solution and checks if some other protein was co-precipitated with it. If this happens it means that the two proteins were either directly interacting with each other or they are part of a multi-protein interaction complex. Both GluN2A and GluN2B receptors was seen to co-immunoprecipitate when PSD-93 was pulled down (Figure 7). This showed that PSD-93 either directly interacts with NMDA receptors or it is physically bound to NMDA receptors via other interacting partner(s). A recent report using Xray crystallography and fluorescence polarization spectroscopic approach, to determine that PSD-93 indeed can interact with NMDA receptor c-terminal tail via its PDZ domain (Fiorentini et al., 2013) also supports my results.

Considerable research has been done to find out the mechanism by which NMDA receptors are internalized. Studies have found a consensus sequence in the $\mathrm{C}$-terminal tail of GluN2B subunits ( $-\mathrm{Y}^{1472}$ EKL-) that binds to the adaptor protein AP-2 (Roche et al., 2001). AP-2 is known to tag proteins having a YXXØ sorting signals (where $\mathrm{X}=$ any amino acid and $\varnothing=$ bulky hydro-phobic amino acid) and mark it for clathrin mediated internalization (Review Traub, 2009). Thus, when AP-2 binds to the cterminal of GluN2B receptors, it gets internalized from the membrane. But when the Tyr-1472 in this internalization domain of GluN2B gets phosphorylated, AP-2 can no longer bind to it and hence, GluN2B cannot be internalized (Roche et al., 2001).

Since in the PSD-93KO animals the surface of level of GluN2B was reduced, I wanted to check whether the phosphorylation status of the GluN2B-Y ${ }^{1472}$ is changed in these animals. I saw that the total level of GluN2B-Y ${ }^{1472}$ phosphorylation does not change in the PSD-93KO animals. This indicates that in absence of PSD-93, the signaling pathway that phosphorylates GluN2B stays unperturbed. But when I checked 
for the surface population of GluN2B, there was a reduction of the Y-1472 phosphorylation in PSD-93KO animals.

This could be because of two possible reasons. First, even if the level of phosphorylated GluN2B does not change, their cellular distribution changes in the PSD-93KO animals. This means that PSD-93 helps in the membrane stabilization of the phosphorylated form of GluN2B molecules. Second, this result could also indicate the Tyr 1472 is not the only site that determines the surface stabilization of GluN2B receptors, as is apparent from the fact that even with similar levels of GluN2B-Y1472 phosphorylation, the sub-cellular distribution changed in PSD-93KO. Interestingly, there are other reported phosphorylation sites on GluN2B (Y1252, Y1336) which are reported to be important for their surface trafficking and stability (Nakazawa et al., 2009; Takasu et al., 2002). The Src Family Kinase, Fyn, phosphorylates these sites (Nakazawa et al., 2009; Takasu et al., 2002). It will be highly relevant to check, if the level of phosphorylation on these sites are reduced in absence of PSD-93. This will further strengthen the idea of PSD-93 as a signaling scaffold, which acts as platform for post-translational modification of other PSD proteins. One other known fact is that the striatal enriched tyrosine phosphatase or STEP, is involved in the removal of Tyr phosphorylation of GluN2B (Salter and Kalia, 2004). I had already shown that in PSD-93 proteins there is higher level of STEP activity than wild-type when NMDA receptors are induced. It is possible that due higher level of STEP in PSD-93, there is lesser phosphorylation of one of the other Tyr sites of GluN2B and hence reduced membrane retention.

As mentioned previously, different reports have different conclusion as to the necessity of PSD-93 in maintaining surface levels of NMDA receptors. One important consideration in between these studies was that PSD-93 showed different roles in different brain regions. So I tried to compare the role of PSD-93 in maintaining NMDA receptor surface levels between hippocampal and cortical dissociated culture models. The cortical cultures showed a reduction in the surface level of GluN2B (Figure 9). There was a trend in the reduction of GluN2A as well, but didn't reach significance. The hippocampal cultures however showed no reduction in the surface levels of either GluN2A or GluN2B levels in PSD-93KO cultures. This again, supported the idea that PSD-93's role is dispensable and redundant in the certain brain regions and some oth- 
Discussion P a g e | 86

er MAGUK may actually compensate for its loss of function (McGee et al., 2001a). But in cortex, PSD-93 is more crucial in maintaining the surface levels of NMDA receptors and its loss causes a reduction in the levels of NMDA receptors, particularly of the GluN2B subunit. 


\subsection{PSD-93 is required for NMDA induced neurotoxicity}

Glutamate induced neurotoxicity is one crucial reason for neuronal death during traumatic brain injuries (Choi, 1988a; Tu et al., 2010a). Owing to their high permeability to $\mathrm{Ca}^{2+}$ ions, NMDA receptors, in particular, are suspected to be responsible in mediating this neurotoxic effect (Lu et al., 2009; Wenthold et al., 1996). Further studies have also indicated that during brain injuries, the release of excess amount of glutamate from the pre-synaptic terminals causes activation of post-synaptic NMDA receptors. This may cause in a rapid influx of $\mathrm{Ca}^{2+}$ ions in the post-synaptic cell. $\mathrm{Ca}^{2+}$ is a well known second messenger system, which then activates pro-apoptotic genes in the cell and causes neuronal death (Sattler et al., 1998). Since, the PSD-93KO animals showed a reduction in the surface expression of NMDA receptors, I investigated the NMDA triggered neurotoxicity in these neurons. If the activation of NMDA receptors is required for neurotoxicity, then the reduction in the surface level of these receptors may reduce the level of neurotoxicity in PSD-93KO animals, as well.

To test this hypothesis, I shifted my experimental model to dissociated neuronal cultures. This was done because it's relatively easy to perform a NMDA challenge experiment in culture. While making the cortical culture, I used whole cortex from WT or PSD-93KO mice, instead of just the visual cortex. Since, changing my experimental model from acute visual cortex punch to cortical dissociated culture was a major paradigm shift, I wanted to first check the levels of NMDA receptors in dissociated culture system as well. The surface level of GluN2B subunit showed a reduction PSD-93KO cortical cultures (Figure 9B).

Reports have claimed that GluN2B receptors are pro-apoptotic in nature (Liu et al., 2007; Tu et al., 2010a). Which means GluN2B receptors, when activated, upregulates down-stream signaling cascades in the cell that leads to apoptosis (Kinases et al., 1999; Waxman and Lynch, 2005b). The level of the activated caspase-3, a serine protease needed for final stages of apoptosis, is classically used to measure the rate of apoptosis in cells (Zhang et al., 2010). When I performed a $30 \mu \mathrm{M}$ NMDA challenge on WT cortical cultures for $1 \mathrm{~h}$ and checked for the levels of activated caspase-3, no detectable signals were found. This could be because that activated 
caspase-3 comes at a very late stage of apoptosis and hence just $1 \mathrm{~h}$ of NMDA receptor activation might not be enough time for the cell to activate caspase-3. So after the $1 \mathrm{~h}$ of NMDA challenge, I incubated the neuronal culture in fresh media for $24 \mathrm{~h}$ and looked at the rate of apoptosis by measuring the levels of activated caspase- 3 . The neurons could show high intensity of cleaved casp-3 under western blot analysis.

Interestingly some recent evidences had come into light, showing that caspase- 3 is present in non-apoptotic cells as well Gilman and Mattson, 2002; Campbell and Holt, 2003; Kuo et al., 2006; Williams et al., 2006; Yuan, 2006). So, to ensure that my NMDA challenge experiment indeed causes neuronal death, I used a PI staining protocol to mark apoptotic cells, post NMDA neurotoxic challenge experiment.

PI staining in my wild-type cells treated with NMDA, showed clear morphological damages to the neuronal membrane. The nucleus of these cells showed characteristic punctate staining pattern, showing nuclear condensation and fragmentation (Figure 11A). When cultures were pre-incubated with APV, which is an antagonist of the NMDA receptors, the apoptotic effects of NMDA could be blocked completely (Figure 11B). This showed that the increase in the level of caspase- 3 in these cells corresponds to apoptotic cell death and is exclusively through the NMDA receptors activation.

When the dose-response curve was plotted for a $1 \mathrm{~h}$ treatment of varying concentrations of NMDA, to check the level of apoptosis in WT cortical cultures, $30 \mu \mathrm{M}$ was identified as a saturating concentration of NMDA for induction of apoptosis (Figure 12). The time kinetics using $30 \mu \mathrm{M}$ NMDA showed that the level of cleaved casp-3 steadily increased until it reached a maximum at $1 \mathrm{~h}$ (Figure 13). But when NMDA is kept on the culture for extended time-points of $4 \mathrm{~h}$ or $8 \mathrm{~h}$, the level of cleaved casp-3 showed a marked reduction. However, when the cells were studied under microscope, they still showed prominent signs of cell damage due to the NMDA treatment. This could have two explanations. One is that when NMDA receptors are activated for $4 \mathrm{~h}$ or $8 \mathrm{~h}$, and then they are allowed to recover in fresh media for a further $24 \mathrm{~h}$, the level of apoptosis is much more advanced than 1h NMDA treatment would cause. At very advanced stages of apoptosis, there are multiple other proteases activated in a cell, which means even the activated caspase-3 might start getting degraded and their lev- 
els may go down. Hence even though these cells had reached advanced stages of apoptosis, the level of activated caspase-3, that could be quantified, is low. The second explanation is that, during sustained activation of NMDA receptors, other caspase independent forms of apoptosis may gets activated and hence, even though the level of caspase-3 is low, non- caspase proteases may might compensate for it (Borner and Monney, 1999; Stoka et al., 2001). But for all further experiments, I used $30 \mu \mathrm{M}$ of NMDA treatment for $1 \mathrm{~h}$ as a standard for my neurotoxicity protocol, as it causes maximal activation of the caspase- 3 dependent cell death pathway, unless mentioned otherwise.

When I performed the NMDA challenge on PSD-93KO cultures, they showed almost an $80 \%$ reduction in the level of cleaved casp-3 (Figure 14). The cells looked healthy under microscopic observation and showed negligible signs of apoptotic cell damage. Since PSD-93 has six N-terminal isoforms, I wanted to check which of them is involved in mediating the NMDA receptor based neurotoxicity. For this reason, I overexpressed these isoforms in a PSD-93KO background. When I checked for the expression level of these PSD-93 over-expression constructs, I found them to be comparable to wild-type level of PSD-93 expression. This is particularly suitable for our study, as over-expressing a protein beyond physiological levels may often lead to unphysiological effects. To make sure that the lentiviral mediated transfection protocol itself didn't cause any neuro-toxicity, I also checked the level of cleaved casp-3 in all the over-expression cultures. None of them showed any detectable amounts of active caspase. When I performed the NMDA neurotoxicity protocol on these cultures, three of the N-terminal isoforms of PSD-93 (PSD-93 $\alpha 1$, PSD-93 $\alpha 2$ and PSD-93 $\beta$ ) could rescue the levels of active casp-3 back to wild-type level. Two of the other isoforms (PSD-93 $\gamma$ and PSD-93e) failed to rescue active casp-3 levels completely, while PSD$93 \delta$ isoform could only partially rescue the neurotoxic effect of PSD-93. Not much is known about differential roles of N-terminal isoforms of PSD-93 in cortical synapses. The only study so far (Krüger et al., 2013b) had shown that in hippocampus, different PSD-93 isoform has differential expression levels and role in maintaining AMPA receptor functions. Thus, it could be likely that even in the cortex, they have differential role in maintaining NMDA receptor surface levels and hence functions differently in the rescue of neurotoxicity. 
Discussion $\mathrm{P}$ a g e $\mid \mathbf{9 0}$

Since NMDA challenge can activate both GluN2A as well as GluN2B subunits of NMDA receptors, I wanted to see the contribution of either of these receptors in the NMDA mediated neurotoxicity in cortex. I used specific pharmacological antagonists

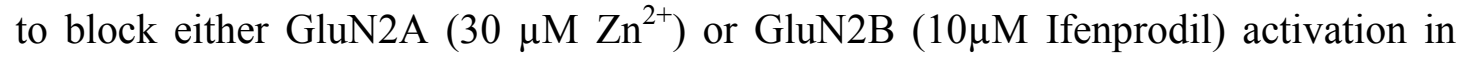
wild-type neurons and then perform a NMDA challenge on them. When GluN2B receptor activation was blocked, there was almost a $80 \%$ reduction in NMDA mediated neurotoxicity (Figure 16B). This is almost in the same range of reduction in neurotoxicity, as was observed in the PSD-93KO neurons. This also follows the current model of understanding that activation of GluN2B is neurotoxic in nature (Liu et al., 2007; Tu et al., 2010a). Blocking of the GluN2A molecule also showed about a small but significant reduction in neurotoxicity (Figure 16A). Generally GluN2A receptors are supposed to be neuro-protective in nature and their blockade had been attributed to increased neuronal mortality (Anastasio et al., 2009; Chen et al., 2008; DeRidder et al., 2006). So the reduction in neurotoxicity, when GluN2A receptors are blocked was unexpected. One explanation of this could be that, $\mathrm{Zn}^{2}$ that was used in this study to block GluN2A subunits is a promiscuous blocker of both GluN2A as well as GluN2B. Although the dose at which I used it, it's supposed to block GluN2A receptors specifically (Izumi et al., 2006), but a non-specific, partial blockade of GluN2B receptors could not be ruled out. In which case the reduction in neurotoxicity may be due to the partially blocked GluN2B receptors and not GluN2A receptors. I can verify the specific effect of GluN2A by RNAi mediated knockdown of the receptor.

The P38 MAPK pathway has been known to be critical in the NMDA mediated neurotoxicity (Barone et al., 2001). So I first I check if this pathway is getting activated in my model of NMDA mediated neurotoxicity. When looked at the time course of P38 activation, I saw an increase in the level of active P38 immediately after 10 min of NMDA treatment, but reached saturation after $30 \mathrm{~min}$ of NMDA treatment (Figure 17). The level of active P38 however came down to untreated control level post $24 \mathrm{~h}$ recovery time. The explanation of this lies in the fact that, P38 is one of the earliest proteins to be activated in the signaling cascade during NMDA induced form of apoptosis. Once active P38 has activated its downstream target proteins like p53, its level goes down and p53 can start activating the further steps of intrinsic apoptotic pathway (Chipuk and Green, 2008; Liu et al., 2003). Then I checked at the level of P38 activation in wild-type vs. PSD-93KO cultures after being treated with NMDA for $1 \mathrm{~h}$. 
Discussion P a g e $\mid 91$

There was a reduction in the level of active p38 in the PSD-93KO cultures (Figure 18). Reports have shown that the Striatal-enriched Tyrosine Phosphate or STEP, also known as PTPN5 down-regulates P38 MAPK activation in neo-cortex by dephosphorylating it (Boulanger et al., 1995). So I checked the level of active STEP in wild-type vs. PSD-93KO neurons. PSD-93KO showed higher levels of active STEP than in wild-type neurons. It is known that upon sustained activation of GluN2B subunit, the influx of $\mathrm{Ca}^{2+}$ ions, at a very high concentration, can activate the $\mathrm{Ca}^{2+}-$ dependent protease m-calpain. Activated m-calpain can cleave active STEP and hence promotes secondary activation of P38 (Xu et al., 2009). Once activated, P38 can causes phosphorylation of the pro-apoptotic transcription factor p53 which activates the intrinsic/mitochondrial pathway of apoptosis (Chipuk and Green, 2008; Liu et al., 2003) (Figure 22). It is most likely that, in PSD-93KO animals due to lower level of surface GluN2B activation, there is much lower level of $\mathrm{Ca}^{2+}$-influx in the postsynaptic cell during NMDA challenge. At such lower concentration of $\mathrm{Ca}^{2+}, \mathrm{m}-$ calpain cannot be activated and hence it cannot cleave STEP to cause a secondary activation of P38. Since P38 remains inactive, it also cannot up-regulate p53 and thus the intrinsic pathway of apoptosis cannot be initiated (Figure 23). 


\section{A. During Basal State}

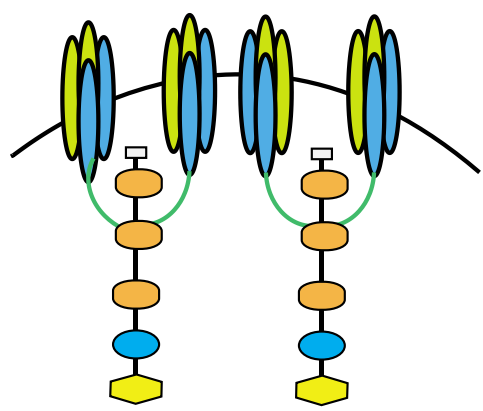

STEP

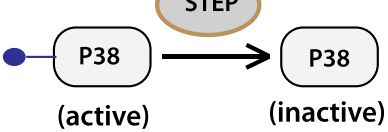

\section{B. During NMDA challenge}
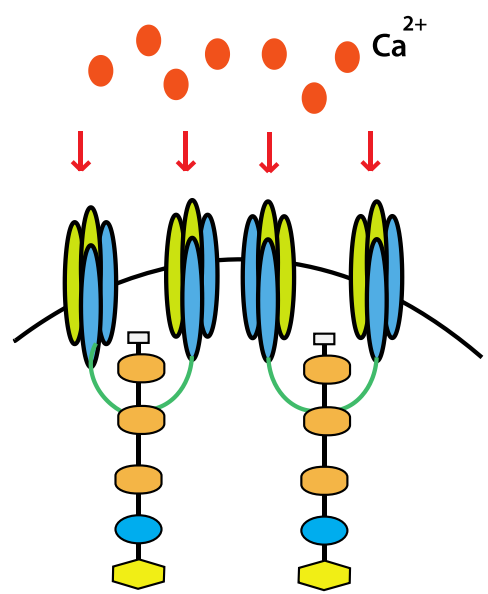

m-calpain<smiles>C=CO</smiles>

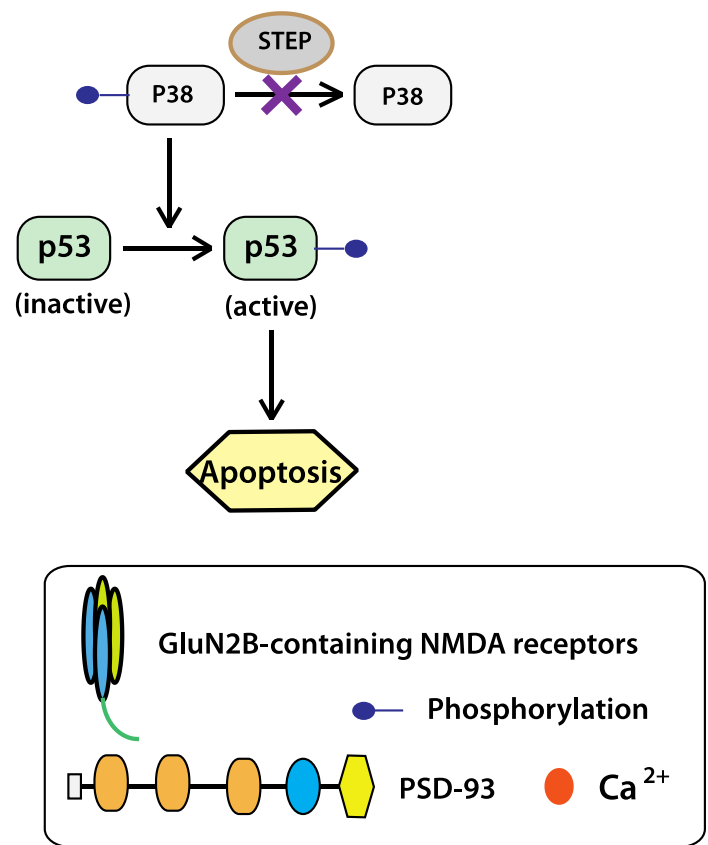

Figure 22: NMDA mediated activation of intrinsic apoptotic pathway in wild-type neurons. A.In basal synaptic state, when NMDA receptors remain inactive, Striatal-enriched Tyrosine Phosphate or STEP (shown in grey) converts active phosphorylated P38 (shown in light grey) into inactive non-phosphorylated state. B. During NMDA mediated neurotoxicity, excess of glutamate released from the pre-synaptic terminal, following ischemic brain injury, leads to activation of NMDARs, especially ones with the GluN2B subunits and causes a rapid influx of $\mathrm{Ca}^{2+}$ (shown in orange) in the cell. At a very high concentration of intracellular $\mathrm{Ca}^{2+}, \mathrm{a} \mathrm{Ca}^{2+}$-dependent protease m-calpain (shown in white) becomes activated that cleaves STEP and causes secondary activation of P38. Active P38 can phosphorylate the pro-apoptotic transcription factor p53 (shown in green), which in turn can initiate the intrinsic/mitochondrial pathway of apoptosis. 


\section{A. During Basal State}

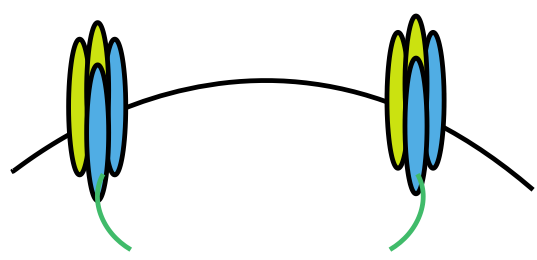

STEP

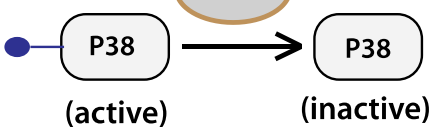

\section{B. During NMDA challenge}

$\mathrm{Ca}^{2+}$

$\downarrow$

$\downarrow$

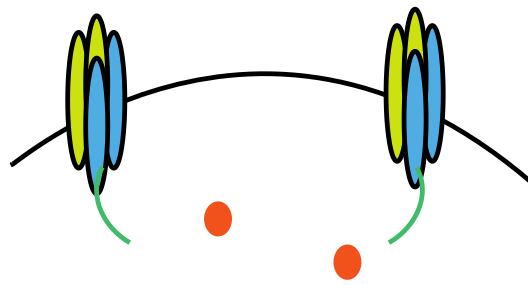

m-calpain

(inactive)

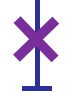

1

STEP
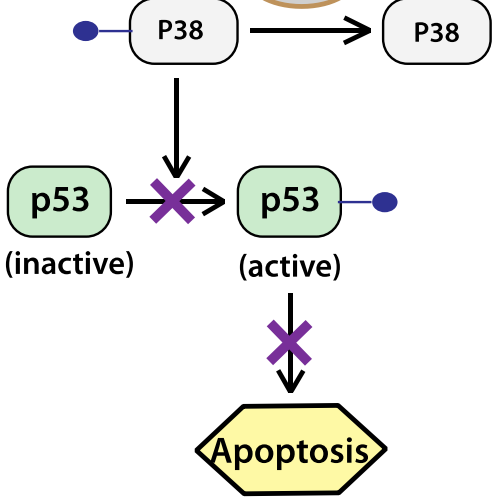

GluN2B-containing NMDA receptors

$$
\mathrm{Ca}^{2+}-\text { Phosphorylation }
$$

Figure 23: In PSD-93KO animals, NMDA mediated activation of apoptosis is blocked. A. In basal condition, PSD-93KO animals have less surface level of GluN2B containing NMDA receptors. STEP keeps P38 protein in its inactive form by de-phosphorylating it. B. During NMDA mediated neurotoxicity, due to reduced level of surface NMDA receptors, low level of $\mathrm{Ca}^{2+}$ ions can enter the post-synaptic cell. At such low level of intracellular $\mathrm{Ca}^{2+}$, the $\mathrm{Ca}^{2+}$-dependent protease m-calpain cannot get activated and hence STEP cannot be cleaved. STEP keeps the P38 in its inactive form and hence blocks all downstream signaling to initiate apoptosis. 


\subsection{Loss of PSD-93 is more neuro-protective than PSD-95 in cortical synapses}

Rapid $\mathrm{Ca}^{2+}$-influx that occurs through the NMDA receptors is the principal cause of neurotoxicity observed during ischemic brain injuries and this neurotoxicity cannot be reproduced by equivalent amount of $\mathrm{Ca}^{2+}$ loading via non-NMDARs and voltagesensitive $\mathrm{Ca}^{2+}$ channels (VSCC) (Sattler et al., 1998). This provides a crucial clue that $\mathrm{Ca}^{2+}$-influx through NMDARs are coupled to specific down-stream signaling pathways that can lead to neurotoxicity. Thus, it was proposed that the neurotoxic $\mathrm{Ca}^{2+}$ signaling through NMDARs are linked to the down-stream signaling cascade by molecules with NMDARs interact (Sattler, 1999a).

PSD-95 is known to interact with NMDA receptors and couple its activation to intracellular signaling cascades (Brenman et al., 1996; Sheng, 2001). PSD-95 is known to interact with neuronal nitric oxide synthase (nNOS), an enzyme that helps nitric oxide formation in neurons (Dawson et al., 1991). The NMDAR-PSD-95-nNOS pathway was found to be the preferential pathway for NO production in neurons over other pathways (Craven and Bredt, 1998; Niethammer et al., 1998). Neuronal NO had long been postulated as a cause of glutamate induced neurotoxicity in neurons and it was found out that PSD-95 helps connect NMDAR mediated $\mathrm{Ca}^{2+}$-influx with NO production and hence promotes neurotoxicity (Sattler, 1999b). This could be further validated by blocking NMDA mediated neurotoxicity by using anti-sense oligonucleotides against PSD-95 (Sattler, 1999b).

As NMDAR-PSD-95-nNOS pathway started being viewed as the principal pathway for NMDA mediated neurotoxicity, this pathway became an intervention target for treating neuronal death caused by NMDA mediated ischemic brain damage. Pharmaceutical intervention by using NMDA receptor antagonists was met with failure as this leads to disruption of crucial physiological functions of NMDA receptors as well (Kemp and McKernan, 2002; Traynor et al., 2006). Suppression of PSD-95 was also an impractical therapeutic strategy, owing to its very important role as being a postsynaptic scaffold molecule. Thus an alternate attempt was made in disrupting the NMDAR-PSD-95 interaction instead. This was done by using an inhibitory peptide 
Discussion $\mathrm{P}$ a g e $\mid 95$

containing nine C-terminal residues of GluN2B (KLSSIESDV; GluN2B9C) (Kornau et al., 1995a) which was made cell permeable by fusing it with the cell-membrane transduction domain of Human Immunodeficiency Virus-1 Tat protein (YGRKKRRQRRR; Tat) (Schwarze, 1999) to obtain the twenty amino acid peptide TatGluN2B9C. Administration of this peptide rendered neurons resistant to focal cerebral ischemia in vivo (Aarts et al., 2002).

Few questions however remain open and unanswered. The C-terminal of GluN2 contains a highly promiscuous PDZ interacting motif (T/SXV), which can interact with several PDZ domain-containing proteins, and any of them could mediate the neurotoxic signaling independent of PSD-95. Of all the several PDZ domain-containing proteins that physically interact with GluN2 receptors, both PSD-95 and PSD-93 are well known. The whole hypothesis that the neurotoxic $\mathrm{Ca}^{2+}$-signaling through NMDARs are linked to the down-stream signaling by MAGUK proteins, only considered PSD-95 and no one has paid much attention to the role of PSD-93 in the same process. Interestingly PSD-93 had been shown to co-localize with NMDA receptors and nNOS in cultured cortical neurons (Xu et al., 2004) and a very recent report also claims that there is lower nNOS mediated cell death in PSD-93KO cortical cultures (Zhang et al., 2010). Also while PSD-93KO animals had been shown to have reduced level of NMDA-dependent persistent pain (Tao et al., 2003). But surprisingly no one had ever looked at occlusion of stroke or NMDA-dependent persistent pain in PSD95KO animals, in spite of it being available for more than a decade (Migaud et al., 1998).

A pull down of GluN2 receptors followed by mass-spectrometric analysis of all the tightly bound protein that was co-immunoprecipitated with it had revealed both PSD95 and PSD-93 as two very strong interactor of GluN2 receptor (Husi et al., 2000). Most surprisingly, Cui et al (2007) had tried to check the binding affinity of TatGluN2B9C peptide with several other PDZ domain containing proteins at an extremely low concentration of the peptide $(0.007 \mu \mathrm{M}$ of Tat-GluN2B9C) to narrow down the subset of strongest interacting partners for this peptide. Both PSD-95 and PSD-93 were seen to bind the peptide with equally strong affinity even at such low concentrations. In the same study, PSD-95 showed a 16-fold higher affinity to bind to GluN2A containing NMDA receptors than GluN2B. Since GluN2A subunit of NMDA recep- 
Discussion $\mathrm{P}$ a g e $\mid 96$

tors are known to be neuro-protective in nature and helps cell survival during CNS injuries (Chen et al., 2008; DeRidder et al., 2006), one may wonder, why deletion of PSD-95, which has a much higher interaction with GluN2A, is neuro-protective in nature? One explanation to this seemingly tricky question could be in the fact that maturity of synapse had been shown to be important in making it susceptible to neurotoxic challenge and immature synapses had been shown to be protected against glutamate-induced toxicity (Choi and Kriegstein, 1987). I had also found it difficult to induce NMDA induced neurotoxicity in cultures that are younger than DIV 17. Overexpression of PSD-95 is known to cause faster maturation of synapses (El-husseini, 2014) whereas, I have already shown that in absence of PSD-95, synapses get arrested in an immature state (Bonnet et al., 2013). Whereas, this definitely is not a case for PSD-93 deletion as synapses have normal development even in it's absence (McGee et al., 2001a) Thus, the neuro-protective effect of PSD-95 deletion might be by keeping the synapses in an immature state while that of PSD-93 might be through other molecular pathway(s).

To check this I first examined the effect that PSD-95 knockdown had on NMDA mediated neurotoxic challenge. Deletion of PSD-95 was seen to be neuro-protective, though to a lesser extent than PSD-93KO cultures (Figure 20). This led me to compare the effect of neuro-protection that PSD-95 knockdown showed in comparison to PSD93 knockout when concentration of NMDA was increased. For the wild-type control culture, I chose $30 \mu \mathrm{M}$ dose of NMDA, as I already had demonstrated it to be a saturating dose of NMDA for maximal activation of caspase-3. For the dose-response curve of NMDA concentration vs. activation of caspase-3 in PSD-93KO vs. PSD-95 knockdown, I used $30 \mu \mathrm{M}$ to $100 \mu \mathrm{M}$ concentration of NMDA. While the deletion of both PSD-93 and PSD-95 were neuro-protective in lower concentration, at higher concentrations of NMDA, PSD-95 knockdown cultures failed to be neuro-protective any more while, PSD-93KO cultures continued showing neuro-protection.

So it is possible that while PSD-95 knockdown does its neuro-protection by arresting the synapse at an immature state and interrupting the NMDAR-PSD-95-nNOS pathway. On the other hand, PSD-93 knockout might actually do its neuro-protection by interrupting both the nNOS and the P38 MAPK activation pathway and hence has increased neuro-protection. 


\subsection{Conclusion and outlook}

The current study helped us understand the role of PSD-93 in maintaining surface level of NMDA receptors in cortical synapses. We could demonstrate that PSD-93 regulates the level of both GluN2A and GluN2B subunit of NMDA receptors while it plays no role in maintaining surface levels of AMPA receptors in the layer $2 / 3$ of visual cortex synapses. Additionally, I showed that GluN2B receptor levels were regulated in PSD-93KO animals independent of its Tyr1472 phosphorylation. The reduction of surface NMDA receptors could also be demonstrated in PSD-93KO cortical cultures, but in hippocampal cultures this reduction was not observed.

I also demonstrated that PSD-93 is required for NMDA mediated neurotoxicity in cortex. It was shown that in absence PSD-93, the P38 MAPK pathway of apoptosis could not be triggered and hence neurons had increased neuro-protection during NMDA receptor mediated neurotoxic challenge.

I could over-express the six different N-terminal isoforms of PSD-93 and identified three of them, which could completely (PSD-93 $\alpha 1, \alpha 2$ and $\beta$ ) rescue the neurotoxicity. As had been shown in hippocampus (Krüger et al., 2013b), possibly these are the most abundant isoforms of PSD-93 in cortex, as well. PSD-93 $\delta$ could only do a partial rescue, which means it might need some other PSD-93 isoform or another MAGUK for its full functionality. The other two isoforms (PSD-93 $\gamma$ and $\varepsilon$ ) failed to rescue neurotoxicity completely.

Lastly, I could demonstrate that deletion of both PSD-95 and PSD-93 showed neuroprotection at low concentrations of NMDA challenge. But at an increased concentration of NMDA challenge, PSD-95 deletion lost its neuro-protective ability, while PSD-93 deletion continued to show its neuro-protection. This demonstrated that in cortical synapses PSD-93 plays a more potent role than PSD-95 in coupling NMDA receptor activation and intra-cellular apoptotic signaling during NMDA neurotoxicity. 
Discussion P a g e $\mid 98$

While our study gives us much knowledge and understanding about the roles of PSD93 in maintenance and physiological properties of NMDA receptors in cortex, there are still some very important aspects to be investigated:

While we know that PSD-93 helps maintain the surface population of NMDA receptors in visual cortex, we still need to identify whether the synaptic, extra-synaptic or entire pool of NMDA receptors is maintained by PSD-93. Some preliminary experiments in our group (personal communication P. Favaro) had indicated that there is no change in the synaptic NMDA currents in visual cortex, which could mean that PSD93 maintains principally the extra-synaptic pool of NMDA receptors. I need to biochemically isolate the synaptic and extra-synaptic fraction of NMDA receptors and determine which fraction has a reduced NMDA receptor in PSD-93KO animals.

We need to check whether the neuro-protective role of PSD-93 in cultures is also valid in an in vivo mice-model of ischemic brain damage .I would also do a comparative analysis between PSD-93 and PSD-95 to see which one has better neuro-protective ability in vivo.

Active caspase- 3 had been shown to be present in cells even in non-apoptotic states known to be involved in cleaving AMPA receptor GluA1 subunit and promote LTD in synapses (Chan et al., 1999; Glazner et al., 2000; Schnell et al., 2002). LTD is not only important in regulating synaptic strength but important for causes spine shrinkage and synaptic elimination called synaptic pruning (Bear et al., 1987; Cramer, 1995; M Constantine-Paton et al., 2003; Ziburkus et al., 2009). Since, PSD-93 has reduced ability to produce active caspase-3 when NMDA receptors are activated, it will be very interesting to check if LTD and synapse numbers are effected in PSD-93KO animals and find out any co-relation with the basal level of active caspase- 3 in these animals. 


\section{References}

Aarts, M., Liu, Y., Liu, L., Besshoh, S., Arundine, M., Gurd, J.W., Wang, Y.-T., Salter, M.W., and Tymianski, M. (2002). Treatment of ischemic brain damage by perturbing NMDA receptor- PSD-95 protein interactions. Science 298, 846-850.

Adesnik, H., and Nicoll, R. a (2007). Conservation of glutamate receptor 2-containing AMPA receptors during long-term potentiation. J. Neurosci. 27, 4598-4602.

Anastasio, N.C., Xia, Y., O'Connor, Z.R., and Johnson, K.M. (2009). Differential role of N-methyl-D-aspartate receptor subunits $2 \mathrm{~A}$ and $2 \mathrm{~B}$ in mediating phencyclidineinduced perinatal neuronal apoptosis and behavioral deficits. Neuroscience 163, $1181-1191$.

Andersen, P., Soleng, a F., and Raastad, M. (2000). The hippocampal lamella hypothesis revisited. Brain Res. 886, 165-171.

Argilli, E., Sibley, D.R., Malenka, R.C., England, P.M., and Bonci, A. (2008). Mechanism and time course of cocaine-induced long-term potentiation in the ventral tegmental area. J. Neurosci. 28, 9092-9100.

Bagal, A. a, Kao, J.P.Y., Tang, C.-M., and Thompson, S.M. (2005). Long-term potentiation of exogenous glutamate responses at single dendritic spines. Proc. Natl. Acad. Sci. U. S. A. 102, 14434-14439.

Barone, F.C., Irving, E. a, Ray, a M., Lee, J.C., Kassis, S., Kumar, S., Badger, a M., Legos, J.J., Erhardt, J. a, Ohlstein, E.H., et al. (2001). Inhibition of p38 mitogenactivated protein kinase provides neuroprotection in cerebral focal ischemia. Med. Res. Rev. 21, 129-145.

Barria, A., and Malinow, R. (2002). Subunit-specific NMDA receptor trafficking to synapses. Neuron 35, 345-353.

Bats, C., Groc, L., and Choquet, D. (2007). The interaction between Stargazin and PSD-95 regulates AMPA receptor surface trafficking. Neuron 53, 719-734.

Bear, M., Cooper, L., and Ebner, F. (1987). A physiological basis for a theory of synapse modification. Science (80-. ). 237, 42-48.

Beckman, J.S., Beckman, T.W., Chen, J., Marshall, P.A., and Freeman, B.A. (1990). Apparent hydroxyl radical production by peroxynitrite: implications for endothelial injury from nitric oxide and superoxide. Proc. Natl. Acad. Sci. U. S. A. 87, 16201624.

Béïque, J.-C., and Andrade, R. (2003). PSD-95 regulates synaptic transmission and plasticity in rat cerebral cortex. J. Physiol. 546, 859-867. 
Béïque, J.-C., Lin, D.-T., Kang, M.-G., Aizawa, H., Takamiya, K., and Huganir, R.L. (2006). Synapse-specific regulation of AMPA receptor function by PSD-95. Proc. Natl. Acad. Sci. U. S. A. 103, 19535-19540.

Bellone, C., and Lüscher, C. (2006). Cocaine triggered AMPA receptor redistribution is reversed in vivo by mGluR-dependent long-term depression. Nat. Neurosci. 9, 636641.

Benveniste, H., Drejer, J., Schousboe, A., and Diemer, N.H. (1984). Elevation of the extracellular concentrations of glutamate and aspartate in rat hippocampus during transient cerebral ischemia monitored by intracerebral microdialysis. J. Neurochem. $43,1369-1374$.

Birnboim, H.C., and Doly, J. (1979). A rapid alkaline extraction procedure for screening recombinant plasmid DNA. Nucleic Acids Res. 7, 1513-1523.

Bonnet, S.A.D. (2011). Role of the Different Domains of PSD-95 in Basal Synaptic Transmission. Georg-August University Göttingen, Germany.

Bonnet, S. a D., Akad, D.S., Samaddar, T., Liu, Y., Huang, X., Dong, Y., and Schlüter, O.M. (2013). Synaptic state-dependent functional interplay between postsynaptic density-95 and synapse-associated protein 102. J. Neurosci. 33, 1339813409.

Borner, C., and Monney, L. (1999). Apoptosis without caspases: an inefficient molecular guillotine? Cell Death Differ. 6, 497-507.

Boudreau, A.C., Milovanovic, M., Conrad, K.L., Nelson, C., Ferrario, C.R., and Wolf, M.E. (2012). A protein cross-linking assay for measuring cell surface expression of glutamate receptor subunits in the rodent brain after in vivo treatments. Curr. Protoc. Neurosci. Chapter 5, Unit 5.30.1-19.

Boulanger, L.M., Wahle, P., and During, M.J. (1995). Cellular and Molecular Tyrosine Phosphatase of a Brain-enriched. 1532-1544.

Bredt, D.S., and Nicoll, R. a (2003). AMPA receptor trafficking at excitatory synapses. Neuron 40, 361-379.

Bredt, D.S., Hwang, P.M., Glatt, C.E., Lowenstein, C., Reed, R.R., and Snyder, S.H. (1991). Cloned and expressed nitric oxide synthase structurally resembles cytochrome P-450 reductase. Nature 351, 714-718.

Brenman, J.E., Chao, D.S., Gee, S.H., McGee, a W., Craven, S.E., Santillano, D.R., Wu, Z., Huang, F., Xia, H., Peters, M.F., et al. (1996). Interaction of nitric oxide synthase with the postsynaptic density protein PSD-95 and alpha1-syntrophin mediated by PDZ domains. Cell 84, 757-767.

Bult, A., Zhao, F., Dirkx, R., Raghunathan, A., Solimena, M., and Lombroso, P.J. (1997). STEP: a family of brain-enriched PTPs. Alternative splicing produces transmembrane, cytosolic and truncated isoforms. Eur. J. Cell Biol. 72, 337-344. 
Campbell, D.S., and Holt, C.E. (2003). Apoptotic pathway and MAPKs differentially regulate chemotropic responses of retinal growth cones. Neuron 37, 939-952.

Cantley, L.C., and Neel, B.G. (1999). New insights into tumor suppression: PTEN suppresses tumor formation by restraining the phosphoinositide 3-kinase/AKT pathway. Proc. Natl. Acad. Sci. U. S. A. 96, 4240-4245.

Carlisle, H.J., Fink, A.E., Grant, S.G.N., and O’Dell, T.J. (2008). Opposing effects of PSD-93 and PSD-95 on long-term potentiation and spike timing-dependent plasticity. J. Physiol. 586, 5885-5900.

Carroll, R.C., Lissin, D. V, von Zastrow, M., Nicoll, R. a, and Malenka, R.C. (1999). Rapid redistribution of glutamate receptors contributes to long-term depression in hippocampal cultures. Nat. Neurosci. 2, 454-460.

Chan, S.L., Griffin, W.S., and Mattson, M.P. (1999). Evidence for caspase-mediated cleavage of AMPA receptor subunits in neuronal apoptosis and Alzheimer's disease. J. Neurosci. Res. 57, 315-323.

Chang, N., El-Hayek, Y.H., Gomez, E., and Wan, Q. (2007). Phosphatase PTEN in neuronal injury and brain disorders. Trends Neurosci. 30, 581-586.

Chen, B.-S., and Roche, K.W. (2007). Regulation of NMDA receptors by phosphorylation. Neuropharmacology 53, 362-368.

Chen, M., Lu, T.-J., Chen, X.-J., Zhou, Y., Chen, Q., Feng, X.-Y., Xu, L., Duan, W.H., and Xiong, Z.-Q. (2008). Differential roles of NMDA receptor subtypes in ischemic neuronal cell death and ischemic tolerance. Stroke. 39, 3042-3048.

Chinnaiyan, a M. (1999). The apoptosome: heart and soul of the cell death machine. Neoplasia 1, 5-15.

Chipuk, J.E., and Green, D.R. (2008). How do BCL-2 proteins induce mitochondrial outer membrane permeabilization? Trends Cell Biol. 18, 157-164.

Cho, K.O., Hunt, C.A., and Kennedy, M.B. (1992). The rat brain postsynaptic density fraction contains a homolog of the Drosophila discs-large tumor suppressor protein. Neuron 9, 929-942.

Choi, D.W. (1988a). Glutamate neurotoxicity and diseases of the nervous system. Neuron 1, 623-634.

Choi, W. (1988b). Pharmacology of Glutamate Neurotoxicity Attenuation by NMDA Antagonists in Cortical Cell Culture : 8 .

Choi, D.W., and Kriegstein, A.R. (1987). Glutamate Neurotoxicity. 7.

Chung, K.K.K., Thomas, B., Li, X., Pletnikova, O., Troncoso, J.C., Marsh, L., Dawson, V.L., and Dawson, T.M. (2004). S-nitrosylation of parkin regulates ubiquitination and compromises parkin's protective function. Science 304, 1328 1331. 
Cohen, G.M. (1997). Caspases: the executioners of apoptosis. Biochem. J. 326 ( Pt 1, $1-16$.

Conrad, K.L., Tseng, K.Y., Uejima, J.L., Reimers, J.M., Heng, L.-J., Shaham, Y., Marinelli, M., and Wolf, M.E. (2008). Formation of accumbens GluR2-lacking AMPA receptors mediates incubation of cocaine craving. Nature 454, 118-121.

Constantine-paton, M., Cline, H.T., and Al, C.E.T. (1990). PATTERNED ACTIVITY , SYNAPTIC CONVERGENCE, AND THE NMDA RECEPTOR.

Cramer, K. (1995). Activity-dependent remodeling of connections in the mammalian visual system. Curr. Opin. Neurobiol. 5, 106-111.

Craven, S.E., and Bredt, D.S. (1998). Synaptic Signaling Pathways. 93, 495-498.

Craven, S.E., El-Husseini, A.E., and Bredt, D.S. (1999). Synaptic targeting of the postsynaptic density protein PSD-95 mediated by lipid and protein motifs. Neuron 22, 497-509.

Cui, H., Hayashi, A., Sun, H.-S., Belmares, M.P., Cobey, C., Phan, T., Schweizer, J., Salter, M.W., Wang, Y.T., Tasker, R.A., et al. (2007). PDZ protein interactions underlying NMDA receptor-mediated excitotoxicity and neuroprotection by PSD-95 inhibitors. J. Neurosci. 27, 9901-9915.

Dakoji, S., Tomita, S., Karimzadegan, S., Nicoll, R. a., and Bredt, D.S. (2003). Interaction of transmembrane AMPA receptor regulatory proteins with multiple membrane associated guanylate kinases. Neuropharmacology 45, 849-856.

Davis, S.M., Lees, K.R., Albers, G.W., Diener, H.C., Markabi, S., Karlsson, G., and Norris, J. (2000). Selfotel in Acute Ischemic Stroke: Possible Neurotoxic Effects of an NMDA Antagonist. Stroke 31, 347-354.

Dawn M., C. (2012). The Balance between Life and Death: Defining a Role for Apoptosis in Aging. J. Clin. Exp. Pathol.

Dawson, V.L., Dawsont, T.E.D.M., London, E.D., Bredtt, D.S., and Snydertt, S.H. (1991). Nitric oxide mediates glutamate neurotoxicity in primary. 88, 6368-6371.

DeRidder, M.N., Simon, M.J., Siman, R., Auberson, Y.P., Raghupathi, R., and Meaney, D.F. (2006). Traumatic mechanical injury to the hippocampus in vitro causes regional caspase-3 and calpain activation that is influenced by NMDA receptor subunit composition. Neurobiol. Dis. 22, 165-176.

Ehrlich, I., and Malinow, R. (2004). Postsynaptic density 95 controls AMPA receptor incorporation during long-term potentiation and experience-driven synaptic plasticity. J. Neurosci. 24, 916-927.

El-husseini, A.E. (2014). PSD-95 Involvement in Maturation of Excitatory Synapses. 1364. 
Elias, G.M., Funke, L., Stein, V., Grant, S.G., Bredt, D.S., and Nicoll, R.A. (2006a). Synapse-specific and developmentally regulated targeting of AMPA receptors by a family of MAGUK scaffolding proteins. Neuron 52, 307-320.

Elias, G.M., Funke, L., Stein, V., Grant, S.G., Bredt, D.S., and Nicoll, R. a (2006b). Synapse-specific and developmentally regulated targeting of AMPA receptors by a family of MAGUK scaffolding proteins. Neuron 52, 307-320.

Fiesel, F.C., Voigt, A., Weber, S.S., Van den Haute, C., Waldenmaier, A., Görner, K., Walter, M., Anderson, M.L., Kern, J. V, Rasse, T.M., et al. (2010). Knockdown of transactive response DNA-binding protein (TDP-43) downregulates histone deacetylase 6. EMBO J. 29, 209-221.

Fiorentini, M., Bach, A., Strømgaard, K., Kastrup, J.S., and Gajhede, M. (2013). Interaction partners of PSD-93 studied by X-ray crystallography and fluorescence polarization spectroscopy. Acta Crystallogr. D. Biol. Crystallogr. 69, 587-594.

Fix, A.S., Horn, J.W., Wightman, K.A., Johnson, C.A., Long, G.G., Storts, R.W., Farber, N., Wozniak, D.F., and Olney, J.W. (1993). Neuronal vacuolization and necrosis induced by the noncompetitive N-methyl-D-aspartate (NMDA) antagonist $\mathrm{MK}(+) 801$ (dizocilpine maleate): a light and electron microscopic evaluation of the rat retrosplenial cortex. Exp. Neurol. 123, 204-215.

Fukata, Y., and Fukata, M. (2010). Protein palmitoylation in neuronal development and synaptic plasticity. Nat. Rev. Neurosci. 11, 161-175.

Futai, K., Kim, M.J., Hashikawa, T., Scheiffele, P., Sheng, M., and Hayashi, Y. (2007). Retrograde modulation of presynaptic release probability through signaling mediated by PSD-95-neuroligin. Nat. Neurosci. 10, 186-195.

Garthwaite, J., Charles, S.L., and Chess-Williams, R. (1988). Endothelium-derived relaxing factor release on activation of NMDA receptors suggests role as intercellular messenger in the brain. Nature 336, 385-388.

Gary, D.S., and Mattson, M.P. (2002). PTEN regulates Akt kinase activity in hippocampal neurons and increases their sensitivity to glutamate and apoptosis. Neuromolecular Med. 2, 261-269.

Giallourakis, C., Cao, Z., Green, T., Wachtel, H., Xie, X., Lopez-Illasaca, M., Daly, M., Rioux, J., and Xavier, R. (2006). A molecular-properties-based approach to understanding PDZ domain proteins and PDZ ligands. Genome Res. 16, 1056-1072.

Gilman, C.P., and Mattson, M.P. (2002). Do apoptotic mechanisms regulate synaptic plasticity and growth-cone motility? Neuromolecular Med. 2, 197-214.

Glazner, G.W., Chan, S.L., Lu, C., and Mattson, M.P. (2000). Caspase-mediated degradation of AMPA receptor subunits: a mechanism for preventing excitotoxic necrosis and ensuring apoptosis. J. Neurosci. 20, 3641-3649.

Graham, D.R.M., Garnham, C.P., Fu, Q., Robbins, J., and Van Eyk, J.E. (2005). Improvements in two-dimensional gel electrophoresis by utilizing a low cost "in- 
house" neutral $\mathrm{pH}$ sodium dodecyl sulfate-polyacrylamide gel electrophoresis system. Proteomics 5, 2309-2314.

Grimm, S., Stanger, B.E.N.Z., and Leder, P. (1996). domain"-containing. 93, 1092310927.

Gu, Z., Kaul, M., Yan, B., Kridel, S.J., Cui, J., Strongin, A., Smith, J.W., Liddington, R.C., and Lipton, S.A. (2002). S-nitrosylation of matrix metalloproteinases: signaling pathway to neuronal cell death. Science 297, 1186-1190.

Hara, M.R., Agrawal, N., Kim, S.F., Cascio, M.B., Fujimuro, M., Ozeki, Y., Takahashi, M., Cheah, J.H., Tankou, S.K., Hester, L.D., et al. (2005). S-nitrosylated GAPDH initiates apoptotic cell death by nuclear translocation following Siah1 binding. Nat. Cell Biol. 7, 665-674.

Hardingham, G.E., Fukunaga, Y., and Bading, H. (2002). Extrasynaptic NMDARs oppose synaptic NMDARs by triggering CREB shut-off and cell death pathways. Nat. Neurosci. 5, 405-414.

He, K., Song, L., Cummings, L.W., Goldman, J., Huganir, R.L., and Lee, H.-K. (2009). Stabilization of Ca2+-permeable AMPA receptors at perisynaptic sites by GluR1-S845 phosphorylation. Proc. Natl. Acad. Sci. U. S. A. 106, 20033-20038.

Hill, M.M., Adrain, C., Duriez, P.J., Creagh, E.M., and Martin, S.J. (2004). Analysis of the composition, assembly kinetics and activity of native Apaf-1 apoptosomes. EMBO J. 23, 2134-2145.

Hollmann, M., and Heinemann, S. (1994). Cloned glutamate receptors. Annu. Rev. Neurosci. 17, 31-108.

Hsu, H., Xiong, J., and Goeddel, D. V (1995). The TNF Receptor I-Associated Protein TRADD Signals Cell Death and NF-KB Activation. 91, 495-504.

Hume, R.I., Dingledine, R., and Heinemann, S.F. (1991). Identification of a site in glutamate receptor subunits that controls calcium permeability. Science 253, 10281031.

Husi, H., Ward, M. a, Choudhary, J.S., Blackstock, W.P., and Grant, S.G. (2000). Proteomic analysis of NMDA receptor-adhesion protein signaling complexes. Nat. Neurosci. 3, 661-669.

Igney, F.H., and Krammer, P.H. (2002). Death and anti-death: tumour resistance to apoptosis. Nat. Rev. Cancer 2, 277-288.

Iguchi, Y., Katsuno, M., Niwa, J., Yamada, S., Sone, J., Waza, M., Adachi, H., Tanaka, F., Nagata, K., Arimura, N., et al. (2009). TDP-43 depletion induces neuronal cell damage through dysregulation of Rho family GTPases. J. Biol. Chem. 284, 22059-22066.

Izumi, Y., Auberson, Y.P., and Zorumski, C.F. (2006). Zinc modulates bidirectional hippocampal plasticity by effects on NMDA receptors. J. Neurosci. 26, 7181-7188. 
Jonas, P., and Burnashev, N. (1995). Molecular mechanisms controlling calcium entry through AMPA-type glutamate receptor channels. Neuron 15, 987-990.

Kaul, M., Garden, G.A., and Lipton, S.A. (2001). Pathways to neuronal injury and apoptosis in HIV-associated dementia. 410.

Kemp, J. a, and McKernan, R.M. (2002). NMDA receptor pathways as drug targets. Nat. Neurosci. 5 Suppl, 1039-1042.

Kerchner, G. a, and Nicoll, R. a (2008). Silent synapses and the emergence of a postsynaptic mechanism for LTP. Nat. Rev. Neurosci. 9, 813-825.

Kinases, M.P., Mukherjee, P.K., Decoster, M.A., Campbell, F.Z., Davis, R.J., and Bazan, N.G. (1999). CELL BIOLOGY AND METABOLISM : Glutamate Receptor Signaling Interplay Modulates Stress-sensitive Glutamate Receptor Signaling Interplay Modulates Stress-sensitive Mitogen-activated Protein Kinases and Neuronal Cell Death *.

Kischkel, F.C., Hellbardt, S., Behrmann, I., Germer, M., Pawlita, M., Krammer, P.H., and Peter, M.E. (1995). Cytotoxicity-dependent APO-1 (Fas/CD95)-associated proteins form a death-inducing signaling complex (DISC) with the receptor. EMBO J. $14,5579-5588$.

Koh, D.S., Burnashev, N., and Jonas, P. (1995). Block of native $\mathrm{Ca}(2+)$-permeable AMPA receptors in rat brain by intracellular polyamines generates double rectification. J. Physiol. 486 ( Pt 2, 305-312.

Kondo, M., Sumino, R., and Okado, H. (1997). Combinations of AMPA receptor subunit expression in individual cortical neurons correlate with expression of specific calcium-binding proteins. J. Neurosci. 17, 1570-1581.

Kornau, H.C., Schenker, L.T., Kennedy, M.B., and Seeburg, P.H. (1995a). Domain interaction between NMDA receptor subunits and the postsynaptic density protein PSD-95. Science 269, 1737-1740.

Kornau, H.C., Schenker, L.T., Kennedy, M.B., and Seeburg, P.H. (1995b). Domain interaction between NMDA receptor subunits and the postsynaptic density protein PSD-95. Science 269, 1737-1740.

Koutsilieri, E., and Riederer, P. (2007). Excitotoxicity and new antiglutamatergic strategies in Parkinson's disease and Alzheimer's disease. Parkinsonism Relat. Disord. 13 Suppl 3, S329-31.

Krüger, J.M., Favaro, P.D., Liu, M., Kitlinska, A., Huang, X., Raabe, M., Akad, D.S., Liu, Y., Urlaub, H., Dong, Y., et al. (2013a). Differential roles of postsynaptic density-93 isoforms in regulating synaptic transmission. J. Neurosci. 33, 1550415517.

Krüger, J.M., Favaro, P.D., Liu, M., Kitlinska, A., Huang, X., Raabe, M., Akad, D.S., Liu, Y., Urlaub, H., Dong, Y., et al. (2013b). Differential roles of postsynaptic 
density-93 isoforms in regulating synaptic transmission. J. Neurosci. 33, 1550415517.

Kruman, I., Bruce-Keller, a J., Bredesen, D., Waeg, G., and Mattson, M.P. (1997). Evidence that 4-hydroxynonenal mediates oxidative stress-induced neuronal apoptosis. J. Neurosci. 17, 5089-5100.

Kuo, C.T., Zhu, S., Younger, S., Jan, L.Y., and Jan, Y.N. (2006). Identification of E2/E3 ubiquitinating enzymes and caspase activity regulating Drosophila sensory neuron dendrite pruning. Neuron 51, 283-290.

Lavezzari, G., McCallum, J., Lee, R., and Roche, K.W. (2003). Differential binding of the AP-2 adaptor complex and PSD-95 to the C-terminus of the NMDA receptor subunit NR2B regulates surface expression. Neuropharmacology 45, 729-737.

Li, Z., Jo, J., Jia, J.-M., Lo, S.-C., Whitcomb, D.J., Jiao, S., Cho, K., and Sheng, M. (2010). Caspase-3 activation via mitochondria is required for long-term depression and AMPA receptor internalization. Cell 141, 859-871.

Lipton, S.A., and Rosenberg, P.A. (1994). Excitatory amino acids as a final common pathway for neurologic disorders. N. Engl. J. Med. 330, 613-622.

Lipton, S.A., Choi, Y.B., Pan, Z.H., Lei, S.Z., Chen, H.S., Sucher, N.J., Loscalzo, J., Singel, D.J., and Stamler, J.S. (1993). A redox-based mechanism for the neuroprotective and neurodestructive effects of nitric oxide and related nitrosocompounds. Nature 364, 626-632.

Lipton, S.A., Nakamura, T., Yao, D., Shi, Z.-Q., Uehara, T., and Gu, Z. (2005). Comment on "S-nitrosylation of parkin regulates ubiquitination and compromises parkin's protective function”. Science 308, 1870; author reply 1870.

Liu, F.-T., Newland, A.C., and Jia, L. (2003). Bax conformational change is a crucial step for PUMA-mediated apoptosis in human leukemia. Biochem. Biophys. Res. Commun. 310, 956-962.

Liu, J., Liu, M.C., and Wang, K.K.W. (2008). Calpain in the CNS: from synaptic function to neurotoxicity. Sci. Signal. 1, re1.

Liu, X.-B., Murray, K.D., and Jones, E.G. (2004). Switching of NMDA receptor 2A and $2 \mathrm{~B}$ subunits at thalamic and cortical synapses during early postnatal development. J. Neurosci. 24, 8885-8895.

Liu, Y., Wong, T.P., Aarts, M., Rooyakkers, A., Liu, L., Lai, T.W., Wu, D.C., Lu, J., Tymianski, M., Craig, A.M., et al. (2007). NMDA receptor subunits have differential roles in mediating excitotoxic neuronal death both in vitro and in vivo. J. Neurosci. $27,2846-2857$.

Lu, W., Shi, Y., Jackson, A.C., Bjorgan, K., During, M.J., Sprengel, R., Seeburg, P.H., and Nicoll, R. a (2009). Subunit composition of synaptic AMPA receptors revealed by a single-cell genetic approach. Neuron 62, 254-268. 
M Constantine-Paton, H T Cline, and, and Debski, E. (2003). Patterned Activity, Synaptic Convergence, and the NMDA Receptor in Developing Visual Pathways.

Malinow, R., and Malenka, R.C. (2002). AMPA receptor trafficking and synaptic plasticity. Annu. Rev. Neurosci. 25, 103-126.

McGee, A.W., Topinka, J.R., Hashimoto, K., Petralia, R.S., Kakizawa, S., Kauer, F.W., Aguilera-Moreno, A., Wenthold, R.J., Kano, M., Bredt, D.S., et al. (2001a). PSD-93 knock-out mice reveal that neuronal MAGUKs are not required for development or function of parallel fiber synapses in cerebellum. J. Neurosci. 21, 3085-3091.

McGee, A.W., Topinka, J.R., Hashimoto, K., Petralia, R.S., Kakizawa, S., Kauer, F., Aguilera-Moreno, A., Wenthold, R.J., Kano, M., and Bredt, D.S. (2001b). PSD-93 Knock-Out Mice Reveal That Neuronal MAGUKs Are Not Required for Development or Function of Parallel Fiber Synapses in Cerebellum. J. Neurosci. 21, 3085-3091.

Migaud, M., Charlesworth, P., Dempster, M., Webster, L.C., Watabe, a M., Makhinson, M., He, Y., Ramsay, M.F., Morris, R.G., Morrison, J.H., et al. (1998). Enhanced long-term potentiation and impaired learning in mice with mutant postsynaptic density-95 protein. Nature 396, 433-439.

Monyer, H., Burnashev, N., Laurie, D.J., Sakmann, B., and Seeburg, P.H. (1994). Developmental and regional expression in the rat brain and functional properties of four NMDA receptors. Neuron 12, 529-540.

Mosbacher, J., Schoepfer, R., Monyer, H., Burnashev, N., Seeburg, P.H., and Ruppersberg, J.P. (1994). A molecular determinant for submillisecond desensitization in glutamate receptors. Science 266, 1059-1062.

Nakagawa, T., Futai, K., Lashuel, H.A., Lo, I., Okamoto, K., Walz, T., Hayashi, Y., and Sheng, M. (2004). Quaternary structure, protein dynamics, and synaptic function of SAP97 controlled by L27 domain interactions. Neuron 44, 453-467.

Nakazawa, T., Shimura, M., Mourin, R., Kondo, M., Yokokura, S., Saido, T.C., Nishida, K., and Endo, S. (2009). Calpain-mediated degradation of G-substrate plays a critical role in retinal excitotoxicity for amacrine cells. J. Neurosci. Res. 87, 14121423.

Niethammer, M., Kim, E., and Sheng, M. (1996). Interaction between the C terminus of NMDA receptor subunits and multiple members of the PSD-95 family of membrane-associated guanylate kinases. J. Neurosci. 16, 2157-2163.

Niethammer, M., Valtschanoff, J.G., Kapoor, T.M., Allison, D.W., Weinberg, R.J., Craig, A.M., and Sheng, M. (1998). CRIPT, a novel postsynaptic protein that binds to the third PDZ domain of PSD-95/SAP90. Neuron 20, 693-707.

Ning, K., Pei, L., Liao, M., Liu, B., Zhang, Y., Jiang, W., Mielke, J.G., Li, L., Chen, Y., El-Hayek, Y.H., et al. (2004). Dual neuroprotective signaling mediated by 
downregulating two distinct phosphatase activities of PTEN. J. Neurosci. 24, 40524060 .

Okamoto, S., Pouladi, M. a, Talantova, M., Yao, D., Xia, P., Ehrnhoefer, D.E., Zaidi, R., Clemente, A., Kaul, M., Graham, R.K., et al. (2009). Balance between synaptic versus extrasynaptic NMDA receptor activity influences inclusions and neurotoxicity of mutant huntingtin. Nat. Med. 15, 1407-1413.

Omori, N., Jin, G., Li, F., Zhang, W.R., Wang, S.J., Hamakawa, Y., Nagano, I., Manabe, Y., Shoji, M., and Abe, K. (2002). Short communication E nhanced phosphorylation of PTEN in rat brain after transient middle cerebral artery occlusion. 954, 317-322.

Passafaro, M., Piëch, V., and Sheng, M. (2001). Subunit-specific temporal and spatial patterns of AMPA receptor exocytosis in hippocampal neurons. Nat. Neurosci. 4, 917-926.

Paul, S., Snyder, G.L., Yokakura, H., Picciotto, M.R., Nairn, a C., and Lombroso, P.J. (2000). The Dopamine/D1 receptor mediates the phosphorylation and inactivation of the protein tyrosine phosphatase STEP via a PKA-dependent pathway. J. Neurosci. $20,5630-5638$.

Paul, S., Nairn, A.C., Wang, P., and Lombroso, P.J. (2003). NMDA-mediated activation of the tyrosine phosphatase STEP regulates the duration of ERK signaling. Nat. Neurosci. 6, 34-42.

Plant, K., Pelkey, K.A., Bortolotto, Z.A., Morita, D., Terashima, A., McBain, C.J., Collingridge, G.L., and Isaac, J.T.R. (2006). Transient incorporation of native GluR2lacking AMPA receptors during hippocampal long-term potentiation. Nat. Neurosci. 9, 602-604.

Poddar, R., Deb, I., Mukherjee, S., and Paul, S. (2010). NR2B-NMDA receptor mediated modulation of the tyrosine phosphatase STEP regulates glutamate induced neuronal cell death. J. Neurochem. 115, 1350-1362.

Porter, a G., and Jänicke, R.U. (1999). Emerging roles of caspase-3 in apoptosis. Cell Death Differ. 6, 99-104.

Pulido, R., Zúñiga, a, and Ullrich, a (1998). PTP-SL and STEP protein tyrosine phosphatases regulate the activation of the extracellular signal-regulated kinases ERK1 and ERK2 by association through a kinase interaction motif. EMBO J. 17, 7337-7350.

Rai, N.K., Tripathi, K., Sharma, D., and Shukla, V.K. (2005). Apoptosis: a basic physiologic process in wound healing. Int. J. Low. Extrem. Wounds 4, 138-144.

Roche, K.W., Standley, S., McCallum, J., Dune Ly, C., Ehlers, M.D., and Wenthold, R.J. (2001). Molecular determinants of NMDA receptor internalization. Nat. Neurosci. 4, 794-802. 
Rodrigues, S.M., Schafe, G.E., and LeDoux, J.E. (2001). Intra-amygdala blockade of the NR2B subunit of the NMDA receptor disrupts the acquisition but not the expression of fear conditioning. J. Neurosci. 21, 6889-6896.

Saelens, X., Festjens, N., Vande Walle, L., van Gurp, M., van Loo, G., and Vandenabeele, P. (2004). Toxic proteins released from mitochondria in cell death. Oncogene 23, 2861-2874.

Saiki, R.K., Scharf, S., Faloona, F., Mullis, K.B., Horn, G.T., Erlich, H.A., and Arnheim, N. (1985). Enzymatic amplification of beta-globin genomic sequences and restriction site analysis for diagnosis of sickle cell anemia. Science 230, 1350-1354.

Salter, M.W., and Kalia, L. V (2004). Src kinases: a hub for NMDA receptor regulation. Nat. Rev. Neurosci. 5, 317-328.

Sambrook, J. (2001). Molecular Cloning: A Laboratory Manual, Third Edition (3 Volume Set). Cold Spring Harb. Lab. Press 13.

Sattler, R. (1999a). Specific Coupling of NMDA Receptor Activation to Nitric Oxide Neurotoxicity by PSD-95 Protein. Science (80-. ). 284, 1845-1848.

Sattler, R. (1999b). Specific Coupling of NMDA Receptor Activation to Nitric Oxide Neurotoxicity by PSD-95 Protein. Science (80-. ). 284, 1845-1848.

Sattler, R., Charlton, M.P., Hafner, M., and Tymianski, M. (1998). Distinct influx pathways, not calcium load, determine neuronal vulnerability to calcium neurotoxicity. J. Neurochem. 71, 2349-2364.

Schlüter, O.M., Schnell, E., Verhage, M., Tzonopoulos, T., Nicoll, R.A., Janz, R., Malenka, R.C., Geppert, M., and Südhof, T.C. (1999). Rabphilin knock-out mice reveal that rabphilin is not required for rab3 function in regulating neurotransmitter release. J. Neurosci. 19, 5834-5846.

Schlüter, O.M., Xu, W., and Malenka, R.C. (2006). Alternative N-terminal domains of PSD-95 and SAP97 govern activity-dependent regulation of synaptic AMPA receptor function. Neuron 51, 99-111.

Schnell, E., Sizemore, M., Karimzadegan, S., Chen, L., Bredt, D.S., and Nicoll, R. a (2002). Direct interactions between PSD-95 and stargazin control synaptic AMPA receptor number. Proc. Natl. Acad. Sci. U. S. A. 99, 13902-13907.

Schwarze, S.R. (1999). In Vivo Protein Transduction: Delivery of a Biologically Active Protein into the Mouse. Science (80-. ). 285, 1569-1572.

Shapiro, A.L., Viñuela, E., and Maizel, J. V (1967). Molecular weight estimation of polypeptide chains by electrophoresis in SDS-polyacrylamide gels. Biochem. Biophys. Res. Commun. 28, 815-820.

Sheng, M. (2001). Molecular organization of the postsynaptic specialization. Proc. Natl. Acad. Sci. U. S. A. 98, 7058-7061. 
Sheng, M., and Kim, E. (2011). The postsynaptic organization of synapses. Cold Spring Harb. Perspect. Biol. 3, 1-20.

Shi, S., Hayashi, Y., Esteban, J. a, and Malinow, R. (2001). Subunit-specific rules governing AMPA receptor trafficking to synapses in hippocampal pyramidal neurons. Cell 105, 331-343.

Simon, R.P., Swan, J.H., Griffiths, T., and Meldrum, B.S. (1984). Blockade of Nmethyl-D-aspartate receptors may protect against ischemic damage in the brain. Science 226, 850-852.

Stein, V., House, D.R.C., Bredt, D.S., and Nicoll, R. a (2003). Postsynaptic density95 mimics and occludes hippocampal long-term potentiation and enhances long-term depression. J. Neurosci. 23, 5503-5506.

Stocca, G., and Vicini, S. (1998). Increased contribution of NR2A subunit to synaptic NMDA receptors in developing rat cortical neurons. J. Physiol. 507, 13-24.

Stoka, V., Turk, B., Schendel, S.L., Kim, T.H., Cirman, T., Snipas, S.J., Ellerby, L.M., Bredesen, D., Freeze, H., Abrahamson, M., et al. (2001). Lysosomal protease pathways to apoptosis. Cleavage of bid, not pro-caspases, is the most likely route. J. Biol. Chem. 276, 3149-3157.

Takasu, M.A., Dalva, M.B., Zigmond, R.E., and Greenberg, M.E. (2002). Modulation of NMDA receptor-dependent calcium influx and gene expression through EphB receptors. Science 295, 491-495.

Tao, Y.-X., and Johns, R.A. (2006). PDZ domains at excitatory synapses: potential molecular targets for persistent pain treatment. Curr. Neuropharmacol. 4, 217-223.

Tao, Y.-X., Rumbaugh, G., Wang, G.-D., Petralia, R.S., Zhao, C., Kauer, F.W., Tao, F., Zhuo, M., Wenthold, R.J., Raja, S.N., et al. (2003). Impaired NMDA receptormediated postsynaptic function and blunted NMDA receptor-dependent persistent pain in mice lacking postsynaptic density-93 protein. J. Neurosci. 23, 6703-6712.

Topinka, J.R., and Bredt, D.S. (1998). N-terminal palmitoylation of PSD-95 regulates association with cell membranes and interaction with $\mathrm{K}+$ channel Kv1.4. Neuron 20, $125-134$.

Tovar, K.R., and Westbrook, G.L. (1999). The incorporation of NMDA receptors with a distinct subunit composition at nascent hippocampal synapses in vitro. J. Neurosci. 19, 4180-4188.

Towbin, H., Staehelin, T., and Gordon, J. (1979). Electrophoretic transfer of proteins from polyacrylamide gels to nitrocellulose sheets: procedure and some applications. Proc. Natl. Acad. Sci. U. S. A. 76, 4350-4354.

Traub, L.M. (2009). Tickets to ride: selecting cargo for clathrin-regulated internalization. Nat. Rev. Mol. Cell Biol. 10, 583-596. 
Traynor, B.J., Bruijn, L., Conwit, R., Beal, F., O’Neill, G., Fagan, S.C., and Cudkowicz, M.E. (2006). Neuroprotective agents for clinical trials in ALS: a systematic assessment. Neurology 67, 20-27.

Tu, W., Xu, X., Peng, L., Zhong, X., Zhang, W., Soundarapandian, M.M., Balel, C., Wang, M., Jia, N., Zhang, W., et al. (2010a). DAPK1 interaction with NMDA receptor NR2B subunits mediates brain damage in stroke. Cell 140, 222-234.

Tu, W., Xu, X., Peng, L., Zhong, X., Zhang, W., Mangala, M., Balel, C., Wang, M., Jia, N., Zhang, W., et al. (2010b). NIH Public Access. 140, 1-24.

Uehara, T., Nakamura, T., Yao, D., Shi, Z.-Q., Gu, Z., Ma, Y., Masliah, E., Nomura, Y., and Lipton, S.A. (2006). S-nitrosylated protein-disulphide isomerase links protein misfolding to neurodegeneration. Nature 441, 513-517.

Vanhoutte, P., and Bading, H. (2003). Opposing roles of synaptic and extrasynaptic NMDA receptors in neuronal calcium signalling and BDNF gene regulation. Curr. Opin. Neurobiol. 13, 366-371.

Vosler, P.S., Brennan, C.S., and Chen, J. (2008). Calpain-Mediated Signaling Mechanisms in Neuronal Injury and Neurodegeneration. 78-100.

Wajant, H. (2002). The Fas signaling pathway: more than a paradigm. Science 296, $1635-1636$.

Walker, D.L., and Davis, M. (2008). Amygdala infusions of an NR2B-selective or an NR2A-preferring NMDA receptor antagonist differentially influence fear conditioning and expression in the fear-potentiated startle test. Learn. Mem. 15, 6774.

Wang, Y., Briz, V., Chishti, A., Bi, X., and Baudry, M. (2013). Distinct roles for $\mu$ calpain and m-calpain in synaptic NMDAR-mediated neuroprotection and extrasynaptic NMDAR-mediated neurodegeneration. J. Neurosci. 33, 18880-18892.

Waxman, E. a, and Lynch, D.R. (2005a). N-methyl-D-aspartate receptor subtype mediated bidirectional control of p38 mitogen-activated protein kinase. J. Biol. Chem. 280, 29322-29333.

Waxman, E. a, and Lynch, D.R. (2005b). N-methyl-D-aspartate receptor subtype mediated bidirectional control of p38 mitogen-activated protein kinase. J. Biol. Chem. 280, 29322-29333.

Wenthold, J., Petralia, S., and Niedzielski, S. (1996). Evidence for Multiple Neurons Complexes in Hippocampal. 76, 1982-1989.

Wenthold, R.J., Prybylowski, K., Standley, S., Sans, N., and Petralia, R.S. (2003). Trafficking of NMDA receptors. Annu. Rev. Pharmacol. Toxicol. 43, 335-358.

Wenzel, a, Fritschy, J.M., Mohler, H., and Benke, D. (1997). NMDA receptor heterogeneity during postnatal development of the rat brain: differential expression of the NR2A, NR2B, and NR2C subunit proteins. J. Neurochem. 68, 469-478. 
Wide-, W., and Kinetics, I.N.T. (1972). APOPTOSIS : A BASIC BIOLOGICAL PHENOMENON WITH WIDE-.

Williams, D.W., Kondo, S., Krzyzanowska, A., Hiromi, Y., and Truman, J.W. (2006). Local caspase activity directs engulfment of dendrites during pruning. Nat. Neurosci. 9, 1234-1236.

Xu, J., Kurup, P., Zhang, Y., Goebel-Goody, S.M., Wu, P.H., Hawasli, A.H., Baum, M.L., Bibb, J. a, and Lombroso, P.J. (2009). Extrasynaptic NMDA receptors couple preferentially to excitotoxicity via calpain-mediated cleavage of STEP. J. Neurosci. 29, 9330-9343.

Xu, Y., Zhang, B., Hua, Z., Johns, R. a, Bredt, D.S., and Tao, Y.-X. (2004). Targeted disruption of PSD-93 gene reduces platelet-activating factor-induced neurotoxicity in cultured cortical neurons. Exp. Neurol. 189, 16-24.

Yao, D., Gu, Z., Nakamura, T., Shi, Z.-Q., Ma, Y., Gaston, B., Palmer, L.A., Rockenstein, E.M., Zhang, Z., Masliah, E., et al. (2004). Nitrosative stress linked to sporadic Parkinson's disease: S-nitrosylation of parkin regulates its E3 ubiquitin ligase activity. Proc. Natl. Acad. Sci. U. S. A. 101, 10810-10814.

Yuan, J. (2006). Divergence from a dedicated cellular suicide mechanism: exploring the evolution of cell death. Mol. Cell 23, 1-12.

Zhang, M., Xu, J.T., Zhu, X., Wang, Z., Zhao, X., Hua, Z., Tao, Y.X., and Xu, Y. (2010). Postsynaptic density-93 deficiency protects cultured cortical neurons from Nmethyl-D-aspartate receptor-triggered neurotoxicity. Neuroscience 166, 1083-1090.

Zhao, M.-G., Toyoda, H., Lee, Y.-S., Wu, L.-J., Ko, S.W., Zhang, X.-H., Jia, Y., Shum, F., Xu, H., Li, B.-M., et al. (2005). Roles of NMDA NR2B subtype receptor in prefrontal long-term potentiation and contextual fear memory. Neuron 47, 859-872.

Zheng, M., Liao, M., Cui, T., Tian, H., Fan, D.-S., and Wan, Q. (2012). Regulation of nuclear TDP-43 by NR2A-containing NMDA receptors and PTEN. J. Cell Sci. 125, $1556-1567$.

Ziburkus, J., Dilger, E.K., Lo, F.-S., and Guido, W. (2009). LTD and LTP at the developing retinogeniculate synapse. J. Neurophysiol. 102, 3082-3090. 


\section{Curriculum Vitae}

\section{Tanmoy Samaddar}

Maschmühlenweg 4, App 104,

37073 Göttingen, Germany
Mobile: +4917670328727

tsamadd@gwdg.de

tanmoysamaddar@gmail.com

\section{Education}

10/2010- Present PhD, Molecular and Cellular Neurobiology

European Neuroscience Institute, Göttingen

08/2007- 07/2009 M.Sc Biotechnology

University of Calcutta, Kolkata, India

07/2004-05/2007 B.Sc (Hons.) Microbiology

University of Calcutta, Kolkata, India 


\section{Research Experiences}

10/2010- Present

Ph.D. at European Neuroscience Institute, Göttingen, Germany

Thesis Title: Role of DLG-MAGUKs in surface NMDAR

localization and its patho-physiological function.

(Supervisor: Dr.Dr. Oliver M Schlüter).

11/2008- 04/2009 M.Sc Project at Department of Biotechnology,

University of Calcutta, Kolkata, India

Thesis Title: The role of $\mathrm{NF- \kappa B}$ signaling in lung cancer model in

mice.

(Supervisor: Prof. Dhrubajyoti Chattopadhay).

04/2008-07/2008 M.Sc. thesis project at National Institute of Immunology,

New Delhi, India.

Thesis Title: Biochemical role of cisplatin on apoptosis in terato-

carcinoma cell lines.

(Supervisor: Dr. Chandrima Shaha). 


\section{Teaching Experiences}

05/2013-06/2013

Supervision of Ms. Sabitha Joseph for Master's project titled

"Olfactory discrimination in Sap97 KD-mice”,

European Neuroscience Institute, Göttingen, Germany.

05/2012-06/2012 Supervision of Ms. Nidhi Suvasini for Master's project titled

'Investigating molecular changes in Dorsal Striatum for cocaine

induced memory formation',

European Neuroscience Institute, Göttingen, Germany.

$11 / 2008$

Tutor at the Second One day Biotech Workshop, Department of

Biotechnology, University of Calcutta, Kalkutta, India.

\section{Expertise}

Cell culture, animal handling (rat/mouse), intra-peritoneal injections of pharmacological agents in rodents, cellular and molecular biology, biochemistry, gene and protein expression analysis, Virus production(AAV and Lenti virus), RNA interference, laser scanning confocal microscopy, Mass spectroscopy, behavioral studies on rodents. 


\section{Scholarships}

11/2013-01/2013 GGNB Ph.D Bridging Fellowship

Göttingen Graduate School for Neurosciences, Biophysics, and

Molecular Biosciences (GGNB), Germany

10/2010- 10/2013 SyMBaD fellowship for Ph.D

Marie Curie $7^{\text {th }}$ framework Initial Training Network

Fellowship, $E U$

08/2009-08/2009 Ph.D scholarship

Indian Institute of Science, Bangalore, India.

07/2009 CSIR/UGC Junior Research Fellowship for Ph.D

Council of Scientific and Industrial Research/University

Grants Commission, New Delhi, India

$04 / 2009$

DBT Junior Research Fellowship for Ph.D

Department of Biotechnology, New Delhi, India 


\section{Organizational skills}

$10 / 2013$

Organized the CMPB retreat in Köln on "Methods in Ph.D”, Köln,

Germany.

$10 / 2011$

Organized the CMPB one day work shop "Bridging microscopy and

Neuroscience”, Göttingen, Germany.

$02 / 2010$

Organized the Indian Institute of Science "Open Science day" from

the department of 'Microbiology and Cell biology', Bangalore, India.

11/2008 Organized the first Biotech Workshop from the 'Department of

Biotechnology, University of Calcutta, Kalkutta, India.

$04 / 2007$

Organized the departmental excursion to the Mother Dairy Pvt. Ltd

company from the Department of Microbiology, D.A.College,

University of Calcutta, Kalkutta, India 


\section{Conference, meetings and workshops}

10/2013 SymBaD Annual Meeting, Stresa, Italy(Talk).

09/2013 CNMPB Retreat, Bad-Sooden Allendorf, Germany.

09/2013 CMPB Student's Retreat, Köln, Germany(Talk).

03/2013 10th Göttingen Meeting of the German Neuroscience Society,

Göttingen, Germany (Joint Poster).

07/2012 Synaptic Basis of Disease conference, Geneva, Switzerland.

07/2012 SyMBaD workshop titled "How to...", organized by CMU,

University of Geneva, Geneva, Switzerland.

10/2011 SyMBaD Annual Meeting, balatonfüred, Hungary(Talk).

10/2011 $\quad 3^{\text {rd }}$ European Synapse Meeting, balatonfüred, Hungary.

04/2011 SyMBaD workshop on "Cell Permeable Peptides", organized by

Department of Pharmacological Sciences, University of Milano,

Milano, Italy.

$10 / 2010$

SyMBaD Annual Meeting, Bordeaux, France. 
02/2010 Indian Institute of Science Open Science Day, Bangalore,

India(Presentation).

$11 / 2008$

$2^{\text {nd }}$ Biotech Worshop, Department of Biotechnology, University of

Calcutta, Kalkutta, India(Teaching).

\section{Publications}

Samaddar T, Favaro P, Schlüter OM. PSD-93: an orchestrator of NMDA receptor function in mice neo-cortex.(in preparation).

Bonnet SA, Akad DS, Samaddar T, Liu Y, Huang X, Dong Y, Schlüter OM. Synaptic statedependent functional interplay between postsynaptic density-95 and synapse-associated protein 102. J Neuroscience. 33:13398- 409

Tripathi R, Samadder T, Gupta S, Surolia A, Shaha C. Anticancer activity of a combination of cisplatin and fisetin in embryonal carcinoma cells and xenograft tumors. Mol Cancer Ther. 10:255-68. 


\section{Personal Information}

Date of Birth: $\quad 15^{\text {th }}$ May, 1986.

Marital Status: $\quad$ Unmarried.

Nationality: $\quad$ Indian.

Languages: $\quad$ English(native speaker), Bengali(native speaker), Hindi(proficient).

Personal Interests: $\quad$ Photography, Travelling, Poetry \& blogging.

\section{References}

Dr.Dr. Oliver Schlüter (Ph.D Thesis Advisor)

Department of Molecular Neurobiologie,

European Neuroscience Institute,

Grisebach Str.5, Göttingen, Germany

Prof. Dr. Michael Hörner (Ph.D. Programme manager)

European Neuroscience Institute,

Grisebach Str.5, Göttingen, Germany. o.schlueter@eni-g.de

Tel: +49 $55139-10374$

Tel: +49 551 39-12307 
Dr.Chandrima Shaha (Master's Thesis Advisor) cshaha@nii.res.in

Director, Tel:+91 99-68-277097

National Institute of Immunology,

Aruna Asaf Ali Marg, New Delhi, India. 